\title{
Against Empathy: Aesthetic-Affective Dimensions of Democratic Deliberation
}

\author{
Mary Frances (Molly) Scudder \\ San Francisco, CA \\ Bachelor of Science, Santa Clara University, 2007 \\ Master of Arts, University of Virginia, 2010
}

A Dissertation Presented to the Graduate Faculty

of the University of Virginia in Candidacy for the Degree of Doctor of Philosophy

Department of Politics

University of Virginia

August, 2014 


\begin{abstract}
Since the deliberative turn in democratic theory, critics and advocates alike have focused on ways to make democratic discourse more inclusive. Taking issue with Jürgen Habermas's narrow understanding of political communication as rational argumentation, scholars such as Iris Young and Lynn Sanders have advocated for incorporating marginalized voices and pluralizing the types of communication permitted in political debate. The prospects for democratic participation are greater than ever, but many participants in this dialogue remain closed off and unreceptive to alternative opinions. This dissertation intervenes in this conversation by highlighting the importance of understanding citizens as listeners and not just speakers. I argue that fostering a receptive disposition on the part of listeners is at least as important for ensuring fair deliberation. In other words, for theories of deliberation to live up to the democratic promise of inclusion, they must account for the dialogically open disposition of citizens that allows them to hear and actually consider alternative points of view.

To this end, this dissertation examines and articulates the receptive disposition required of citizens engaging in democratic deliberation. I also consider potential ways that this disposition can be brought about. For example, I explore the role that empathy can play in bridging distances between participants in deliberation. In recent years, theorists such as Michael Morrell and Sharon Krause have lauded the benefits of empathy in helping citizens communicate and gain understanding across differences. To them, empathy refers to both cognitive role taking as well as affective feelings of empathic concern. I reconstruct accounts of the democratic value of empathy and show the limits of an empathy approach to deliberation. Engaging with social scientific research, I show that empathy is not only very difficult to achieve in practice, but also tends to displace the more pressing need for improved listening. Empathy represents a premature and illusory consensus that undermines the very possibility of some eventual agreement by diverting our energy from the need to actually enter into real discourse in the first place. Citizens do not need to listen to each other if they presume understanding can be gained by simply imagining another's point of view.

I argue that greater attention to differences, rather than commonalities, helps dampen the presumption of knowing another person's perspective. Recognizing differences instead of commonalities maintains a theoretical focus on the challenges and limits to mutual understanding. The challenges to democratic discourse in a pluralistic world are significant, given that it occurs always already in the presence of disagreement. Given the magnitude of these challenges, it is crucial that citizens be aware of them. If these challenges are ignored in favor of an approach that focuses primarily on similarities and our ability to imagine and understand another's feelings and motivations, citizens are not alerted to the vital importance of engaging in rich, complex, and sometimes difficult listening practices.

The approach that I develop in this dissertation as an alternative to empathy focuses on helping citizens recognize differences as well as the limits to mutual understanding created by those differences. Recognizing the cultural, experiential, and communicative divides between ourselves and others can help foster more inclusive democratic practices by attuning citizens to the ways we remain closed off to each other as well as the urgent need for improved listening.
\end{abstract}




\section{Table of Contents}

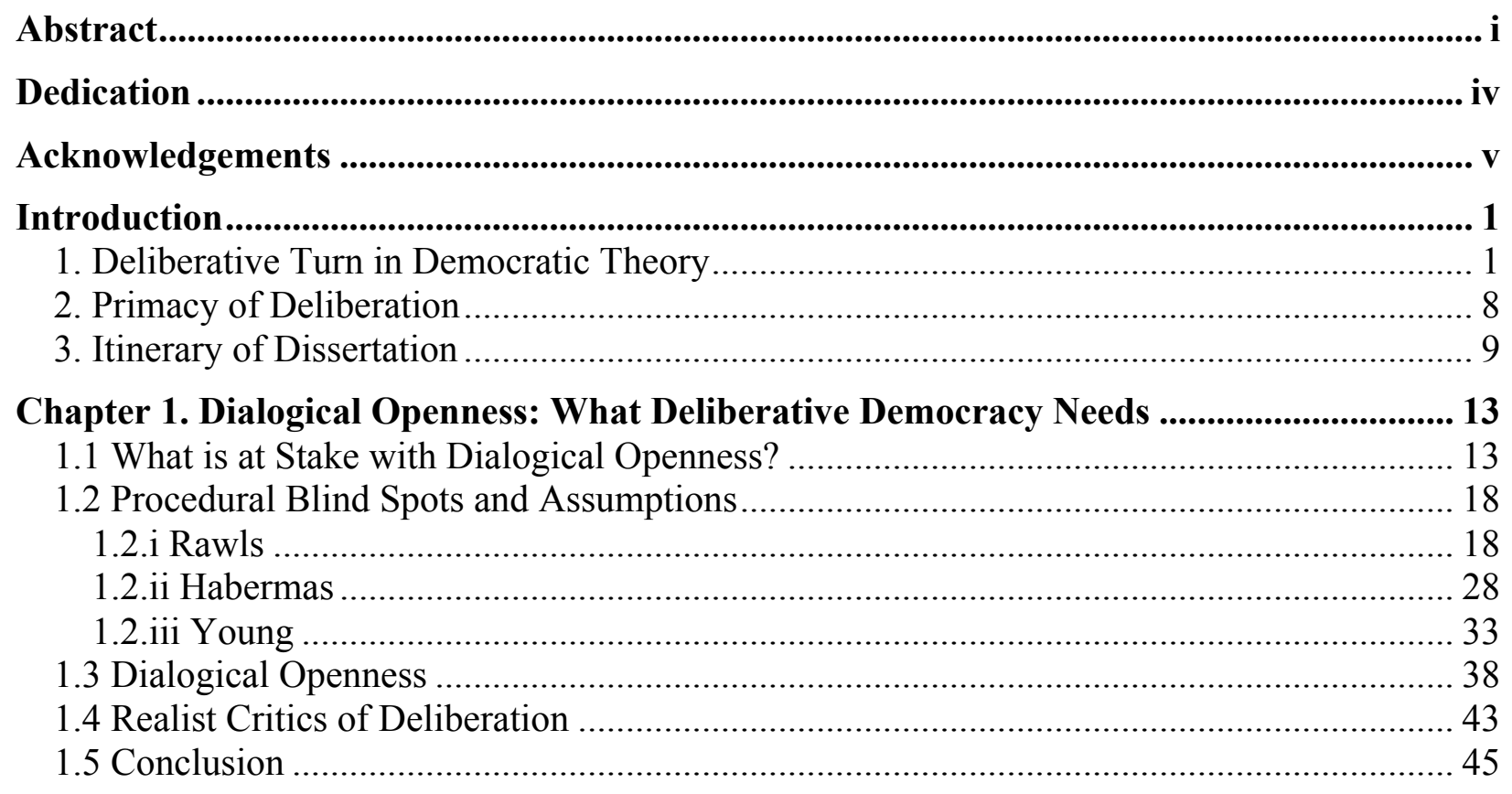

Chapter 2. Beyond Empathy: Strategies and Ideals of Democratic Deliberation .................. 47

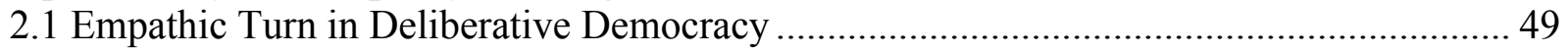

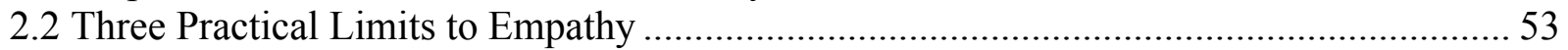

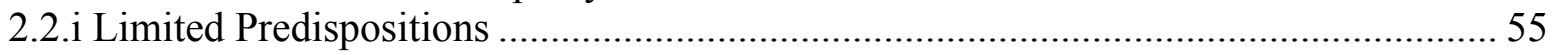

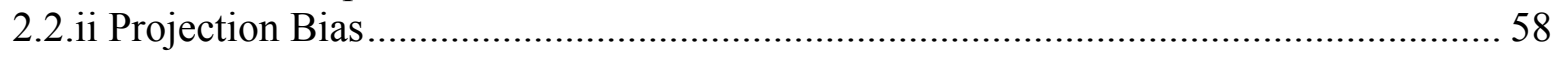

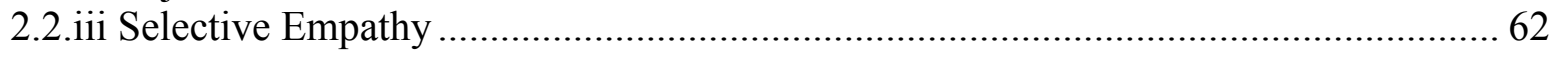

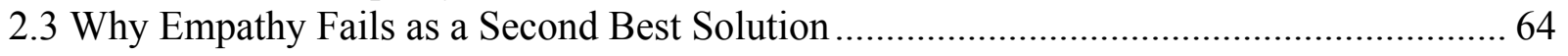

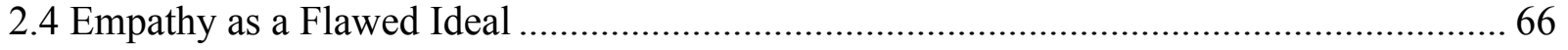

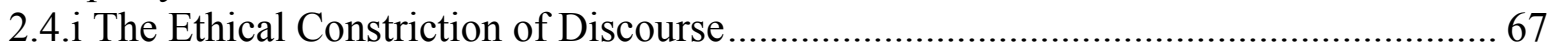

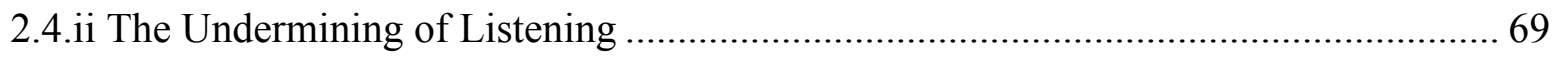

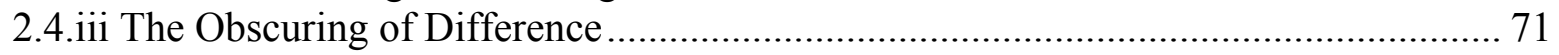

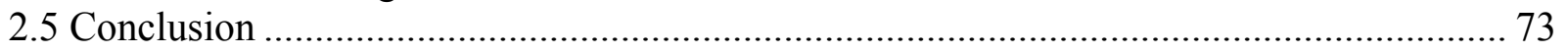

Chapter 3. The Difference Approach to Improving Democratic Deliberation ........................ 75

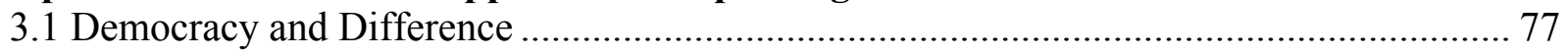

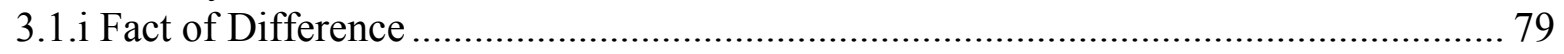

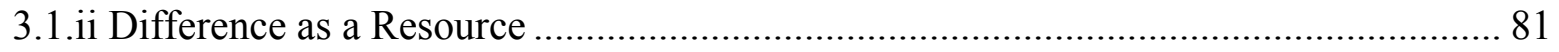

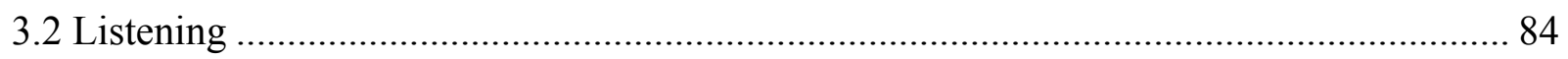

3.3 The Difference Approach …………………………..................................................... 89

3.3.i Martin Heidegger, an Unlikely Resource for Democratic Theory .............................. 91

3.3.ii Humility and the Provisionality of Perspective ................................................................ 96

3.3.iii Receptivity through the Recognition of the Other..................................................... 98

3.3.iv Generosity of a Delayed Response to Difference ...................................................... 100

3.4 The Difference Approach Compared to the Empathy Approach....................................... 101

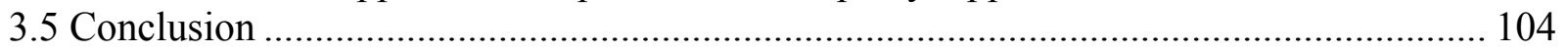


Chapter 4. Aesthetic Sources of Dialogical Openness .............................................................. 106

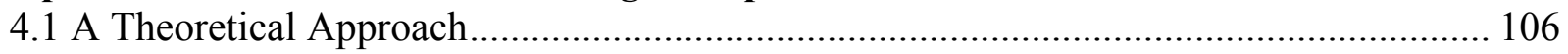

4.2. Art and Politics ...................................................................................................... 109

4.2.i The Dangers of Aesthetics and the Promise of Reason........................................... 110

4.2.ii The Dangers of Reason and the Promise of Aesthetics ............................................. 113

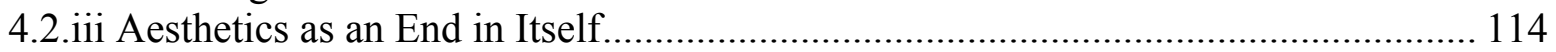

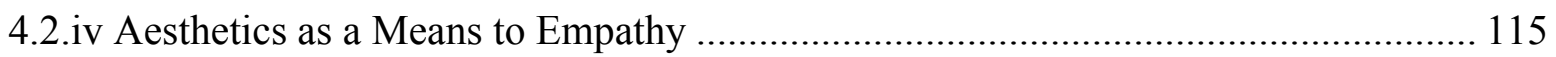

4.3 Aesthetics and the Cultivation of Dialogical Openness................................................... 118

4.3.i Multiplicity and the Provisionality of Beliefs........................................................... 119

4.3.ii Reception and Recognition through Disruption ................................................... 121

4.3.iii Delayed Response, the Sublime, and Generosity ................................................. 125

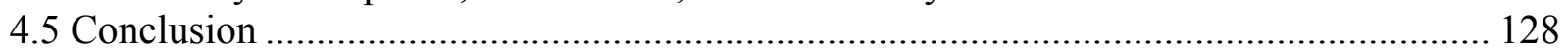

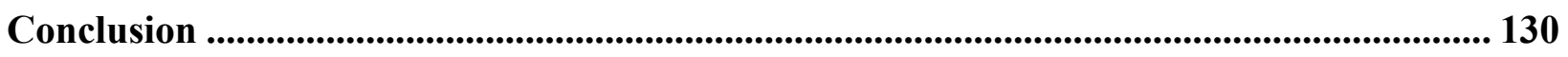

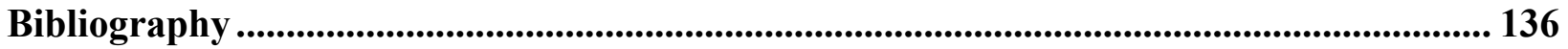




\section{Dedication}

For my greatest teachers, John and Judy Scudder.

And for Kyle Haynes, the most dialogically open person I know. 


\section{Acknowledgements}

The ideas and arguments presented in this dissertation have developed over the course of my six years at the University of Virginia. Naturally, I have amassed many intellectual debts along the way. I am happy to acknowledge them here. In particular, I would like to thank my committee chair, Stephen K. White for the insightful and always constructive criticism that he has provided over the last six years. There could be no more generous advisor than he, who read and re-read multiple drafts. I am also immensely grateful to my committee members, Lawrie Balfour and George Klosko for their help, advice, and criticism over the last six years. My dissertation would be much poorer if it were not for their suggestions.

Much appreciation is owed to Jen Rubenstein and Lynn Sanders as well as to my fellow graduate students who participated in their graduate development seminars in 2011 and 2012. The seeds of my dissertation were tested and nurtured during that time. I am deeply indebted to the generosity that Jen has shown since, reading papers and offering comments long after I was enrolled in her class. I am also grateful to Colin Bird who gave generously of his time and enthusiasm despite my never having had the pleasure to work with him in a formal capacity.

I am grateful to Clarissa Hayward and Frank Lovett at Washington University in St. Louis for welcoming me into their political theory community, and inviting me to present at their Political Theory Workshop. The comments I received at the workshops were instrumental in the development of my critique of empathy. I thank especially Ron Watson and Matt Chick for their feedback as well as their friendship.

I would not have been able to complete this project without the financial and intellectual support from the Institute for Advanced Studies in Culture at the University of Virginia. Lunches with my friends at the IASC were always energizing and stimulating. I thank especially Chad 
Wellmon for his constructive comments at the 2014 Spring Fellows Colloquium and for giving of his time to serve on my committee.

I owe a great deal to my friends and fellow graduate students, past and present. My dissertation and life would be significantly less rich without Regev Ben Jacob, Emily Charnock, Callum Ingram, Nadim Khoury, Colin Kielty, Kyle Lascurettes, Dave Novitsky, Emily Pears, Hilde Restad, Kate Sanger, Greta Snyder, Emily Sydnor, Claire Timperley, Will Umphres, and Brandon Yoder, as well as the original Salonerati: Anne Daniels, Evan Farr, Ellie Kaknes, and Derek King.

Certainly my largest debt is owed to my family, especially my parents John and Judy Scudder. As educators, they endowed my sister and me with a great curiosity for learning. I thank my sister Annie who happily suffered my company from elementary school to college (and ultimately the same political science department), as well as my parents who have supported me through all twenty-three years of formal education. I am forever grateful for their encouragement and unyielding confidence. Their concern and selfless service for others inspires me in both my life and my work.

And last, but not least, I am forever indebted to Kyle Haynes for his love, support, and editing (of course, all grammatical errors are his). I would be remiss if I did not acknowledge the admiration I have for the seemingly effortless way in which he modeled the path to dissertation completion. I thank him for putting up with me during the stress of this last year and for proposing marriage even then! I dedicate my dissertation to him with gratitude and love. 


\section{Introduction}

That man is much more a political animal than any kind of bee or any herd animal is clear. For, as we assert, nature does nothing in vain; and man alone among the animals has speech. The voice indeed indicates the painful or pleasant, and hence is present in other animals as well...But speech serves to reveal the advantageous and the harmful, and hence also the just and the unjust.

\section{-- Aristotle, Politics}

\section{Deliberative Turn in Democratic Theory}

According to Aristotle, it is our capacity for speech that sets humans apart as zoon politikon, or political animals. Humans alone have the gift of speech. With this capacity, we are able to live together politically, coming to collective judgments about what is just and unjust.

This dissertation explores the relationship between speech and justice in contemporary democratic politics. Specifically, I join conversations about democratic speech that have appeared after the sharp deliberative turn taken in democratic theory in the last three decades. Since the deliberative turn, models of democratic deliberation have proliferated (Bessette, 1980; Manin 1987; Cohen 1989; Rawls 2005; Habermas 1996; Young 2001; Dryzek 2002; Goodin 2003). Despite the veritable diversity of each model, deliberative democrats in general share certain fundamental claims that set them apart from other kinds of democrats. With this turn, "the essence of democracy itself is now widely taken to be deliberation, as opposed to voting, interest, aggregation, constitutional rights, or even self-government" (Dryzek 2002, 1). Rather than "mere majority rule," democracy is said to depend on "the means by which a majority comes to be a majority" (Tilden, quoted by Dewey 1988 [1927], 207). John Dewey famously 
explains that the essential need for democracy "is the improvement of the methods and conditions of debate, discussion and persuasion" (Dewey 1988 [1927], 207).

Despite this shared focus on the methods and conditions of debate, discussion, and persuasion, deliberative democrats do not all agree about what it means for citizens to reach their collective decisions through deliberation. Deliberation remains a contested concept in democratic theory.

Are decisions to be judged on purely procedural grounds, or is there a substantive element to democratic legitimacy? In other words, if fair procedures lead to decisions that violate individual liberties or human rights, do deliberative democrats have recourse to challenge the outcomes independent of procedure? Furthermore, if we accept an extensive procedural element to the model of deliberative justice, what do these procedures entail? Although I cannot resolve these questions in my dissertation, let alone the Introduction, I hope to shed light on productive ways to begin answering these questions. I would argue that many of the debates and disagreements in the deliberative democracy literature gain much of their momentum from the ambiguity of the word deliberation.

To deliberate comes from the Latin word, to weigh. In English, the verb has two related, though different, meanings. The Merriam-Webster dictionary conflates the two meanings of to deliberate in its definition: "to think about or discuss something very carefully in order to make a decision (my emphasis)." In our everyday understanding of the word, people can deliberate through thought or speech. Deliberation can occur both within and without, independently and collectively (Goodin 2000). 
The Oxford English Dictionary parses out these two meanings in its definition of to deliberate. Definition 1A in the Oxford English Dictionary does not mention communication between individuals. Instead to deliberate is defined as 'to weigh in the mind; to consider carefully with a view to decision; to think over." Definition $2 \mathrm{~B}$, on the other hand, offers a definition in relation to "a body of persons" as "to take counsel together, considering and examining the reasons for and against a proposal or course of action." Only with this second definition is there any mention of deliberation without, understood as communication for the purpose of coming to a collective decision.

I would contend that much of the lasting disagreement and debate over democratic deliberation can be traced back to these two understandings of deliberation: deliberation-asthought, or reflection, and deliberation-as-speech, or communication. While some deliberative democrats emphasize the value of thoughtful consideration and reflection on the part of citizens, others point to actual speech and communication between citizens as the vital contribution of deliberative theories of democracy. These two meanings, though connected, have vastly different implications for theories of deliberative democracy.

To clarify this confusion, I offer a distinction between deliberate democracy on the one hand and deliberative democracy on the other. Where the former stresses the importance of encouraging citizens to base decisions on considered judgments and reflective preferences, the latter emphasizes the inextricability of democracy and communication among citizens. For deliberative democrats, speech is the quintessential democratic act. In contrast, for deliberate democrats, the goal is not speech or discourse, per se, but rather consideration and reflection on the part of citizens. At times, self-identified deliberative democrats appear to endorse more of a 
deliberate view, as when John Dryzek claims that authentic democracy occurs to the extent that "reflective preferences influence collective outcomes" $(2002,2)$.

Goodin and Niemeyer, recognize the two meanings of deliberation when they explain that political deliberation involves both "internal reflection and public discussion" $(2003,627)$. Furthermore, they find evidence suggesting that "deliberation within...[might be] a more important part of the process than the dialogic and discursive element that is so cherished by contemporary deliberative democrats" $(2003,628)$. To these deliberate democrats, deliberation within is more important to the "overtly political processes" of decision-making than deliberation without (or deliberation as speech).

Goodin and Niemeyer come to this conclusion after analyzing deliberations of a citizen's jury. They show that “jurors' attitudes [change] more in response to the 'information' phase of the jury proceedings, involving a large degree of 'deliberation within', than during the formal 'discussion' phase” (2003, 627). Insofar as citizens changed their attitudes and opinions on a topic by focusing their attention to it, collecting information about it, and being invited "to think hard about it," Goodin and Niemeyer commend them for being reflective and deliberate in their opinion formation $(2003,642)$. By finding that people's opinions are formed and constituted more by these pre-discursive processes of internal reflection (deliberation as thought) than by the processes of discourse (deliberation as speech), they conclude that internal deliberation is more effective at bringing about deliberate, thoughtful, and reflective opinions and decisions. If the deliberate-ness of decisions is the priority, and if this deliberate-ness can be heightened by encouraging citizens to be more internally reflective, than we can improve democracy even in the absence of public discussion. Goodin and Niemeyer prioritize the maximization of deliberate 
decisions, rather than inherently deliberative decisions. In fact, Goodin terms his own model of deliberative democracy, Reflective Democracy (2003).

As I aim to show here, Goodin and Niemeyer as well as other "deliberate democrats" (including Ackerman, Fishkin, and Estlund) ignore perhaps the most essential claim in favor of deliberation as speech: namely, the normative value of communication among citizens. Understood in this way, deliberation is no longer a means of improving democracy, it is constitutive of it.

For deliberative rather than deliberate democrats, especially Jürgen Habermas, actual communication is not a means to an end, but an end in itself. This communication may have desirable effects, insofar as it transfers information and, as a result, improves the epistemic value of collective decisions. This communication will also encourage, if not force, careful consideration within, as individual citizens are called to provide reasons for their opinions, preferences, and decisions. But more importantly, or more fundamentally, reaching decisions through communicative practices ensures that individuals participate in the authorship of the laws to which they are held. Only through deliberation as speech can the democratic promise of autonomy be realized. Communication is not just a means to encourage greater reflection, but the realization of democracy itself.

As Habermas explains, "The idea of self-legislation by citizens, that is, requires that those subject to law as its addressees can at the same time understand themselves as authors of law. We cannot meet this requirement simply by conceiving the right to equal liberties as a morally grounded right that the political legislator merely has to enact" (Habermas 1996, 120). Selflegislation is enacted to the extent that administrative power is responsive to and reflective of the 
"communicative power" generated by informal and diffuse deliberations in the public sphere. "The flow of communication between public opinion-formation, institutionalized elections, and legislative decisions is meant to guarantee that influence and communicative power are transformed through legislation into administrative power" (Habermas 1996, 299). With this transformation, collective opinion is reflected in the collective will. The facticity of the law meets with validity; and positive law becomes legitimate law. This understanding of deliberative democracy maintains citizens' "rational motives for obeying the law: it must remain possible for everyone to obey legal norms on the basis of insight" (Habermas 1996, 121).

According to Habermas, "the discursive level of public debates constitutes the most important variable" $(1996,304)$. But the level of discursiveness "must not be hidden away in the black box of an operationalization satisfied with crude indicators." (Habermas 1996, 304). Discursiveness cannot be operationalized as the transformation of opinion (as in Goodin and Niemeyer's study).

The "self" of democratic self-rule is not a collection of subjects who are internally reflective and deliberate, but rather the "subjectless will," formed only out of the intersubjective communication between all citizens. According to Habermas, "The 'self' of the self-organizing legal community disappears in the subjectless forms of communication that regulate the flow of discursive opinion- and will-formation in such a way that their fallible results enjoy the presumption of being reasonable" (Habermas 1996, 301). Therefore, there is no subject whose internal deliberations we can measure to guarantee deliberation is reflective and deliberate.

With the help of Habermas, we see that models of "democratic deliberation" depart from the deliberative ideal to the extent that they prioritize the internal reflection of citizens over 
actual communication between citizens. "This is not to denounce the intuition connected with the idea of popular sovereignty but to interpret it intersubjectively" (Habermas 1996, 301).

Analogous to the lexical priority of the Liberty Principle over Fair Equality of Opportunity and the Difference Principle defended by John Rawls in A Theory of Justice, I contend that deliberation (as in actual communication between citizens) is lexically prior to deliberate-ness (or internal reflection, deliberation within). If deliberation within ever conflicts with deliberation without, our democratic commitment urges us to prioritize deliberation without. Deliberation as speech cannot be sacrificed for greater levels of internal reflection. Despite the lexical priority of deliberative-ness over deliberate-ness, it would be meaningless to value communication if it was not itself reflective. When talking about reflective communication, however, we can only refer to its intersubjective quality. You raise a validity claim, and I consider it. If I accept the claim, we continue with our communication. If I am unable to accept your validity claim, we enter into discourse to find the source of our disagreement and resolve it if possible. Reflection and deliberate-ness occurs externally and intersubjectively.

Rather than maximize the deliberate-ness or reflectiveness of individual citizens, which is difficult if not impossible to operationalize without introducing an external standard such as the "rationalization" of opinion, we should aim to maximize the intersubjective or discursive level of actual communication among citizens. To this end, we must focus on maximizing the inclusiveness of democratic deliberation as speech, understanding inclusion to mean both participation and consideration. 
Deliberative democracy seeks to maximize both inclusiveness of public communication (to generate communicative power) as well discursiveness of democratic decisions (or the extent to which communicative power is translated into administrative power). The democratic power of communication lies in its intersubjective quality. Therefore, we must continually search for ways to improve the practices and procedures of political communication. With this view, the focus of deliberative democrats must be the assurance that deliberation is open to the offering and criticizing of validity claims from all segments of the population. Generally speaking, this is the task that I take up in this dissertation.

\section{Primacy of Deliberation}

In separating out these two meanings of deliberation, I aim to demonstrate two distinct claims that are often spun together in deliberative theories of democracy. To make a decision deliberately is not the same as to make a decision deliberatively. Can these values come into conflict? If they do, which value should we prioritize? If reflection can occur in the absence of actual communication among citizens, then what is the value of deliberation as speech? Furthermore, what if communication or debate among citizens does not promote greater reflection? We have all had conversations with people who are not particularly good listeners, or whose intransigence and confidence in their own opinion undermine any contribution that your point of view can make to their opinions. In these situations we might choose to "save our breath." Is communication valuable only to the extent that it improves reflection? If we find other, non-communicative ways to induce reflection, such as empathy or imagined perspective taking, should we adopt these procedures instead? Whether you are a deliberate or a deliberative democrat will determine how you answer these questions. 
With Habermas, I contend that we cannot dispense with communication among citizens, no matter how effectively we can induce deliberate-ness and reflection through means other than deliberation as speech. Public discourse is not a stepping-stone to a higher value. It is the highest value. As most people would accept, democracy cannot tolerate a philosopher king. Furthermore, deliberation is not simply a means of achieving a collective philosopher king. Deliberation has value as an end in itself. Our commitment to democracy prohibits us from sacrificing democratic communication to ensure more deliberate decisions.

\section{Itinerary of Dissertation}

Engaging critically with what is, broadly speaking, the Habermasian understanding of democratic deliberation explained above, this dissertation asks how citizens can be drawn in the direction of greater open-mindedness when engaging in democratic discourse. If public discourse carries the promise of democracy, how can we ensure that the discourse is inclusive of all perspectives and opinions? I contribute to democratic theory by searching for new answers to the problem of political inequality and exclusion in democratic deliberation. To date, my fellow deliberative democrats have focused primarily on making deliberation (as speech) more inclusive by incorporating marginalized voices and pluralizing the types of communication permitted in political debate. Missing, however, are considerations of how citizens can become open, receptive listeners when encountering the perspectives and demands of others. My project makes a novel contribution to democratic theory and the issue of political exclusion by considering the important question of democratizing citizen uptake rather than just increasing or 
pluralizing input. ${ }^{1}$ The prospects for democratic participation are greater than ever, but many participants in this dialogue remain closed off and unreceptive to alternative opinions.

Fitting form to content, this dissertation proceeds as a series of conversations. In each chapter, I take on a different set of questions, but more importantly a different set of interlocutors with whom I consider (as dialogically as possible for a dissertation) the question of improving democratic discourse among citizens in pluralistic polities. The theme that animates and unites these conversations is the aesthetic-affective dimensions of inclusion and receptivity in democratic deliberation.

In Chapter 1, I speak to three of the most prominent deliberative democrats, namely Rawls, Habermas, and Iris Young. To ensure that deliberation is deliberate, reflective, and intersubjective, we must attend to the question of dialogical openness among citizens. Recent decades have seen numerous inquiries into how democratic discourse can be made more inclusive. Accounting for a receptive disposition on the part of listeners is crucial when considering how to include marginalized voices in deliberation. Any theory of deliberation must attend to what I call "dialogical openness"- a disposition of greater humility, receptivity, and generosity on the part of citizens engaging in deliberation. Yet, as I explain in Chapter 1, this part of the puzzle is often ignored.

To correct for this omission, I examine and articulate the receptive disposition required of citizens engaging in democratic deliberation. The prospects for fair and equal deliberation are greatly improved when citizens show humility, receptivity, and generosity to their fellow

\footnotetext{
${ }^{1}$ Although uptake is in many ways internal to individual citizens, it occurs always already within actual communication. In this way, it differs markedly from the internal reflection or deliberation within that deliberate democrats emphasize.
} 
citizens. Such a disposition among citizen-listeners allows for a more searching and multidimensional consideration of political questions. Though necessary for deliberation to occur, dialogical openness is not inherent to the process itself. Citizens must cultivate it. In later chapters, I consider potential ways that this disposition can be brought about.

In Chapter 2, I speak to political theorists who explore the role of affect in democracy, proposing empathy as a solution to the problem that I identify as dialogical closure. Though I am sympathetic (no pun intended) to their attempt to make citizens more reflective and receptive in their communicative encounters with others, I argue that their specific intervention misses the mark. Specifically, I challenge the widespread view that empathy should function as a means to bridge distances between participants in deliberation. By empathy, I refer to both cognitive role taking and affective feelings of concern for others. I show that empathy is not only very difficult to achieve in practice, it also tends to displace the more pressing need for improved listening. Empathy may align with the goal of greater deliberate-ness, but at the expense of actual deliberative-ness.

In Chapter 3, I develop the "difference approach" as an alternative to the empathy approach. In this development, I speak to "difference democrats," who like myself point to the ways in which models of democratic deliberation fail to produce reflection on and consideration of minority perspectives and dissenting views. Inclusion in communication has two sides. Voices must be permitted in actual communication, but we must also look for ways to ensure that dissenting and minority voices are actually heard and considered. Calling on Martin Heidegger's later writing on the limits to mutual understanding inherent to language, I offer a new understanding of the fact of difference in democratic deliberation. I intervene in this conversation 
by highlighting the importance of understanding citizens as listeners and not just speakers. The potential of difference to improve discourse expands if we understand citizens in their role as listeners as well as speakers. I show that difference can serve as a useful resource in promoting listening and "uptake" in democratic encounters. Greater attention to differences, rather than commonalities, helps dampen the presumption of knowing another person's perspective. Specifically, the recognition of difference can alert citizens to the limits of mutual understanding and the need for improved listening in light of those limits. The "difference approach" I propose in Chapter 3 cultivates the virtue of modesty, helping citizens understand the hard work required by democratic listening and decision-making.

Lastly, in Chapter 4, I turn to the question of how the recognition of differences can be fostered so as to generate dialogical openness through the "difference approach." In other words, how might we enact the difference approach in practice? How can citizens be made to recognize and gain respect for the differences that exist among democratic co-citizens? Here, I explore how exposure to artistic expression, especially works of aft that have a disruptive effect on viewers and are multiplicitous in their meanings and interpretations, explicitly highlight the perpetual concealedness that remains between citizens. These qualities of certain works of art can invite citizens to become more open in their interactions with others. In this final chapter, my interlocutors include those who have addressed the relevance and appropriateness of art in politics.

I opened the Introduction with Aristotle's assertion that speech gives humans the capacity to consider matters of justice. Building on the claim regarding the centrality of speech, this dissertation explores the correlate of speech, listening. 


\section{Chapter 1. Dialogical Openness: What Deliberative Democracy Needs}

In deliberative democratic theory, the openness that deliberation requires is assumed rather than solicited or adequately theorized. This assumption is deeply problematic in part because it distances deliberative theory from a political reality where people often resist listening to alternative viewpoints and remain closed off to those who are different from them. Deliberation among citizens who are closed off to each other fails to achieve the "promise inherent in democracy," namely that "before a society makes decisions that it will use its collective power to enforce, it will give equal consideration to everyone in the community" (Morrell 2010,1). As a result, this closure has the potential to threaten the justificatory power of those decisions. Dialogical openness is what I interpret to be the disposition required of citizens engaging in democratic discourse. People who are dialogically open are those citizen-listeners who show a significant degree of humility, receptivity, and generosity when engaging with others.

\subsection{What is at Stake with Dialogical Openness?}

Promoters and detractors alike have pointed to the ways in which models of democratic deliberation fall short in both theory and practice. One of the main criticisms leveled against democratic theory since its deliberative turn relates to its inability to accommodate the deep differences and disagreements that exist in politics. Those who offer this kind of criticism of democratic deliberation can be broadly referred to as "difference democrats." According to John Dryzek, "Difference democrats are those who stress the need for democratic politics to concern itself first and foremost with the recognition of the legitimacy and validity of the particular perspectives of historically-oppressed segments of the population" $(2002,57)$. Sharing this commitment, difference democrats take issue (to varying degrees) with democratic deliberation. 
I understand the category of "difference democrat" to transcend the typical categories of agonistic or deliberative democrats. Although all agonistic democrats are probably rightly understood as difference democrats, not all difference democrats are agonistic democrats. Some difference democrats, such as William Connolly or Chantal Mouffe dispense with the deliberative ideal altogether, endorsing a more agonistic understanding of democracy. Others, including Iris Marion Young, aim to make deliberative practices more accommodating of deep difference.

In general, the criticisms and concerns regarding deliberative democracy that are offered by difference democrats can be grouped into three main categories. Though they often appear side by side, I offer the following categories for analytical purposes.

The first category is made up of those who take issue with the means of deliberation, which most often include a narrow understanding of rational argumentation. These critics argue that rational argument can be "coercive and exclusive" (Dryzek 2002, 57). By permitting only certain kinds of reasons and restricting the types of communication in public discourse, models of democratic deliberation run the risk of excluding certain groups and individuals while privileging others. For example, Iris Young and Lynn Sanders both contend that permitting only rational argumentation undermines the goal of neutrality in deliberation and actually favors those in power, including white men, while excluding groups that use other means of communicating such as emotional speech or rhetoric (Sanders 1997, Young 2000).

Similarly, those who take issue with the consensual ends of deliberation argue that the single-minded drive toward consensus and agreement creates "remainders" who are ultimately ignored and excluded from discussion. According to these critics, the deliberative ideal of 
consensus, even when pursued with the best of means, crowds out difference and disagreement (Connolly 1995, Mouffe 2000, Sanders 1997).

In the third category are those who take issue with the conditions of deliberation, or what critics identify to be inherent power asymmetries present in society at the time of deliberation. Until the unequal social and political conditions of citizens are ameliorated, deliberation will fail to produce the legitimate outcomes many of its advocates believe it should. Sanders, for example, claims that given the unequal and power-laden conditions of deliberation specifically and politics generally, fair and equal deliberation is a naïve, unattainable, and misguided goal (1997). Still others point to the inextricability of power and politics and have searched instead for ways that democracy can cope with the inevitably unequal and non-ideal conditions of democratic discourse (Shapiro 2003). These critics contend that our energies should be directed toward designing institutions that redistribute power (specifically decision-making power) rather than aimed at ensuring and guaranteeing access to communicative venues (Shaprio 2003).

Since the deliberative turn, procedural corrections have been proposed for problems related to the means, ends, and conditions of deliberation. Given the procedural nature of deliberative democracy, it is not surprising that so much attention has been paid to tinkering with the very procedures that are expected to produce just outcomes. A marked focus has been on designing procedures that pluralize the voices included in deliberation as well as amplify the dissenting voices that remain after a decision has been made. These kinds of corrections have proven fruitful terrain for making deliberation more democratic and inclusive.

But in order to ensure inclusive uptake and not just input in democratic deliberation and to guarantee that people not only have the chance to speak but also to be heard, we must account 
for a receptive disposition on the part of citizen-listeners. To this end, I go beyond the discussion of procedures of speaking to incorporate procedures and conditions for greater listening. For deliberatively legitimate decisions to be made, citizens must listen to one another with a significant degree of humility, receptivity, and generosity. This listening, however, cannot be achieved or even approximated by simply amplifying the voices of relevant parties.

For all of these reasons, I contend that an account of the disposition or attitude required of citizens in order to sustain capacious listening is one of the most important components of a successful model of deliberative democracy. Developing our understanding of the very dialogical openness that is required and often merely assumed on the part of participants is crucial if we are to realize successful deliberation. If unaddressed, the deficit of dialogical openness runs the risk of derailing even the most inclusive and democratic procedures. Despite the importance of this disposition, it has largely been ignored in three of the most comprehensive approaches to democratic deliberation ${ }^{2}$ within large, diverse societies - those of John Rawls, Jürgen Habermas, and Iris Young. ${ }^{3}$

In order to make the means, ends, and conditions of deliberation more hospitable to difference, we must take into account the importance of listening and the extra-procedural obstacles to as well as facilitators of improved listening. Without an account of dialogical

\footnotetext{
${ }^{2}$ The attention to affect and disposition has appeared primarily in the work of agonistic democrats rather than deliberative democrats. The exception to this dearth of research regarding affect and deliberation is the relatively recent literature regarding the role that empathy should play in deliberation. In Chapter 2, I address the shortcomings of this approach to improving democratic deliberation, arguing that empathy does not further dialogical openness.

${ }^{3}$ Young's work occupies a unique position in democratic theory. Young can perhaps best be described as a skeptical proponent of deliberation. For that reason she appears in this chapter as well as the dissertation as both a critic and an advocate of democratic deliberation.
} 
openness, including both its nature (discussed below) and potential sources (which I discuss in Chapters 2-4), theories of deliberation will remain unable to accommodate deep difference. That is not to say that the problems raised by the critics discussed above can be traced back to a lack of dialogical openness alone. Rather, I claim that greater dialogical openness can at least make us more aware of and attentive to these concerns.

Difference and disagreement cannot be removed from politics, and should at times be celebrated and protected. Citizens, however, must be able to deliberate in the presence of these differences. Theorizing the kind of disposition that would make citizens more open to listening to each other is of vital importance. We must understand dialogical openness at a conceptual and theoretical level before we can point to the ways that we might actually achieve it in practice. Only with this understanding can we pursue a more equitable and fair consideration of all perspectives, given conditions of moral conflict, including scarcity, limited generosity, incompatible values, and incomplete understanding, (Gutmann and Thompson 25).

In Section 1.2, I show how deliberative democrats, despite their efforts to design fair deliberative procedures, have remained blind to obstacles that impede free, equal, and inclusive deliberation even in a context of procedural perfection. Examining the work of Rawls, Habermas, and Young, I identify two features that lead them to systematically ignore the need for dialogical openness. First, as we see in the work of Rawls, the cultivation of dialogical openness is replaced with formal procedural guarantees of fairness and inclusion. As I demonstrate, however, these procedural guarantees fail when they are applied to informal deliberative settings. Second, as evidenced in the work of Habermas and Young, dialogical openness is often assumed to be an inherent feature of communication in both formal and 
informal deliberative institutions and procedures. But dialogical openness is often absent; and this weakens the very procedures these theorists aim to develop, as it obscures our understanding of the conditions necessary for those procedures to succeed.

Section 1.3 gives an account of the concept of dialogical openness and explains how it helps to fulfill the promise of deliberative democracy. Such an account gives us a better understanding of the conditions necessary for deliberatively legitimate decisions to be made across difference. Building on the development of this concept, the remainder of the dissertation compares possible sources of this dialogical openness, exploring the most fruitful ways to foster it among citizens.

\subsection{Procedural Blind Spots and Assumptions}

\section{2.i Rawls}

Given the fact of reasonable pluralism - or the fact that citizens in a liberal democracy subscribe to different and often incompatible metaphysical and religious beliefs (what Rawls calls comprehensive doctrines) - how can citizens reach decisions that are deemed legitimate by all? To explain how citizens might reach agreement in spite of their diverse commitments and beliefs, Rawls offers "the idea of public reason." Perhaps paradoxically, Rawls maintains that in order to accommodate and respect the diversity of comprehensive doctrines among citizens, these differences must be excluded from deliberation (Rawls 2005, 216). In Political Liberalism, Rawls explains that "the ideal of citizenship imposes a moral, not a legal, duty - the duty of civility - to be able to explain to one another on those fundamental questions how the principles and policies they advocate and vote for can be supported by the political values of public reason" $(2005,217)$. According to Rawls, legitimate decisions are those made and defended by appealing 
to only "public reasons" - those supported by the shared public political culture. Excluded from the public political forum are comprehensive reasons rooted in religious or metaphysical beliefs, which cannot be assumed to be shared by all reasonable citizens.

Much has been written in regards to whether Rawls's idea of public reason is too restrictive or even necessary. Can citizens achieve neutrality when discussing questions of basic justice and constitutional essentials? And is this neutrality even desirable (McCarthy 1994)? Critics have also taken issue with Rawls's singular view of public reason. For example, Bohman (1996) argues that Rawls's idea of public reason is unnecessarily restrictive and should be pluralized. Others argue that the idea of public reason is not neutral and, in fact, favors some comprehensive doctrines over others. For example, secularists may have an easier time satisfying the limits of public reason than theists who, as a result, would have a unique and unfair burden when engaging in public deliberation.

Rawls addresses many of these critics by pointing to the circumscribed application of public reason. In "The Idea of Public Reason Revisited," he specifies that he is only concerned about debates over constitutional essentials or questions of basic justice. Furthermore, Rawls points out that the limits of public reason apply only to formal deliberations in the public political forum and not informal communicative interactions in the "background culture." He explains that "sometimes those who appear to reject the idea of public reason actually mean to assert the need for full and open discussion in the background culture. With this political liberalism fully agrees" (1997, 768).

In this section, I do not critique or even fully engage with Rawls's idea of public reason. Instead, I want to consider what he misses by focusing primarily on the public political forum in 
the first place — namely the informal and diffuse communicative interactions among citizens that take place outside of formal decision-making bodies. In his treatment of public reason, Rawls sidesteps any in-depth consideration of the conditions for just deliberation in the background culture. I argue that this omission is problematic because what he leaves aside, deliberation in the background culture, is extremely important for answering questions regarding the establishment of fair procedures of democratic discourse.

Setting aside the limits of public reason as such, I am interested here in their application. Are the limits of public reason required by the particular setting or by the content of the question at hand? The answer is both. Rawls clearly states that the idea of public reason applies to debates of constitutional essentials and questions of basic justice that take place in the public political forum. In sum, the limits of public reason are required by both the content and setting of deliberation. Rawls, however, is not always clear or consistent in distinguishing the two conditions that trigger the need for public reason.

At times, the idea of public reason seems to apply to all deliberations among citizens in regard to relevant subject matter (i.e. constitutional essentials and questions of basic justice) regardless of where they take place. For example, Rawls says that even in a representative government wherein citizens do not directly choose the laws, citizens would ideally "think of themselves as if they were legislators and ask themselves what statutes, supported by what reasons satisfying the criterion of reciprocity, they would think it most reasonable to enact" (1997, 769). Here Rawls suggests that when considering fundamental questions, even when that consideration will not lead directly to enforceable laws, citizens have a moral duty to justify their positions using only public reasons. This reading would extend the limits of public reason into 
the background culture when relevant subject matter is discussed. Accordingly, we have reason to believe that the limits of public reason apply anytime questions of basic justice or constitutional essentials are discussed, regardless of the deliberative setting. I will call this reading the "expansive interpretation."

Take for example deliberation over the question of abortion. If the legality of abortion is debated among members of a church or on a cable news talk show should individuals be expected to appeal only to public reasons? The expansive interpretation would suggest that when discussing the legality of abortion (rather than its morality or permissibility for a member of particular Church) the limits of public reason should apply. ${ }^{4}$

There remains, however, strong textual evidence in favor of rejecting this expansive interpretation in favor of a narrower one. The narrow interpretation holds that Rawls intends for the limits of public reason to be applied only to the public political forum and never to the background culture. Rawls clearly states that: "the idea of public reason does not apply to the background culture with its many forms of nonpublic reason nor the media of any kind" (1997, 768). Deliberation in the background culture occurs outside of any formal decision-making body. In these informal deliberative encounters, whether during a talk show or a religious service, citizens are free to appeal to comprehensive reasons. According to Rawls, using comprehensive reasons in these settings does not amount to trying to impose one's views on another because no decision will be made; no law will be passed as a result of these informal deliberations. Therefore, there is no risk involved with allowing citizens to appeal to their comprehensive

\footnotetext{
${ }^{4}$ There is some debate as to whether the question of abortion amounts to a question of basic justice or a constitutional essential. Rather than defend the status of the question of abortion, I will note that Rawls himself uses this example.
} 
reasons. According to the narrow interpretation of public reason, the background culture is always open to public and nonpublic reasons alike. Citizens are expected and encouraged to debate a wide range of positions and beliefs that are based on shared public reasons as well as unshared comprehensive reasons.

Given Rawls's insistence that the limits of public reason do not apply to the background culture, the text seems to best support the narrow interpretation. Public reason then applies only to deliberation in the public political forum. I argue, however, that there remain theoretical reasons (if not textual ones) to believe that the expansive view is more in line with Rawls's stated goals.

Communication among citizens in the background culture must be incorporated into any complete account of fair and inclusive democratic deliberation. It is vital for understanding the anatomy of legitimate democratic decisions. Rawls's omission of this component - according to the narrow interpretation of public reason - leads to serious weaknesses in his model of democratic deliberation because it limits our understanding of the complete process of legitimate decision-making. Despite being supported by the text, the narrow interpretation of public reason fails to legitimize coercive decisions in the way that Rawls hopes it will.

After showing that the narrow interpretation of public reason ignores the question of fair deliberation in the background culture, I go on to argue that the expansive interpretation fails to ensure it. Despite the limits of the narrow interpretation, I am unable to endorse the expansive view of public reason. Applying public reason to the background culture is impossible. In regards to ensuring fair deliberation across difference in the background culture, we must look 
beyond procedure and give an account of the kind of disposition required of citizens engaging in deliberation.

Importance of Background Culture

By separating the background culture from the political forum, Rawls implicitly acknowledges two distinct phases of democratic deliberation: collective opinion-formation followed by collective will-formation. Before a collective will is formed in the public political forum, where citizens, judges, and legislators decide the best course of action based on shared public reasons, a collective opinion is formed in the background culture. Legitimate decisions are reached only if both phases of deliberation are inclusive of and open to all citizens. And yet in the narrow interpretation of public reason, Rawls concerns himself only with the public political forum, and therefore only with procedures of collective will-formation. By leaving behind deliberation in the background culture, Rawls does not discuss legitimating procedures for collective opinion-formation in the background culture. If Rawls implicitly acknowledges these two phases of deliberation, why does he ignore the former and discuss only the latter?

Rawls downplays the initial, informal phase of deliberation in his model not because he thinks it is unimportant, but because he mistakenly identifies it as free from coercion. The outcomes of deliberation in the opinion-formation stage are not immediately binding or coercively enforced. ${ }^{5}$ Therefore, under the narrow interpretation of public reason, deliberations

\footnotetext{
${ }^{5}$ This assessment of deliberation in the background culture being non-coercive aligns well with John Dryzek's discussion of cool deliberation, or deliberation that is decoupled from formal decision-making bodies. According to Dryzek cool deliberative settings are the most fruitful setting for political debate in divided societies where intractable divisions often derail formal deliberative engagement, let alone, consensus or agreement. Cool deliberative settings are attractive because people can deliberate without threat of "losing" and being forced to abide by the winner's decisions.
} 
in these settings do not need to be held to the same standards as deliberation in the public political forum. ${ }^{6}$

The expansive interpretation of public reason challenges this assessment of the public political forum as uniquely coercive. Deliberation in the background culture is removed from the coercive decisions of the public political forum only by time, not substance. For example, debates about abortion that occur on a cable news show or in a university classroom do not occur in a vacuum. The effects that these conversations have on coercive decisions may be indirect, but they are real. Deliberative encounters in the background culture precede formal deliberation in the public political forum. Despite being pre-procedural in this literal sense, these diffuse and informal encounters play a significant role in generating the collective decisions that are ultimately reached in the public political forum. ${ }^{7}$ Rawls's aim to prevent anyone from forcing his own comprehensive doctrines on others could lead him to support the expansive view of public reason.

Even if we accept Rawls's understanding of democratic deliberation as culminating in a final decision-making moment, that moment is always preceded by deliberation occurring "across wide distances and over long times, with diverse social sectors speaking to one another across differences of perspective as well as space and time" (Young 2000, 46). Unless this

\footnotetext{
${ }^{6}$ Whether the limits of public reason are required in order to legitimize coercion or to ensure coauthorship (as in Bird's view), they are not applied to the background culture because the background culture is seen as separate and disconnected from official political decisions (in this case separate from both coercion or authorship).

${ }^{7}$ For example, the flood of referenda and judicial decisions in favor of marriage equality has been attributed to major shifts in public opinion regarding same-sex marriage over the last decade (Lax \& Phillips 2009, 2012).
} 
informal communicative process is open, free, and inclusive, the binding decision made in the political forum will lack democratic legitimacy.

A process of collective will-formation that is cut off from the preceding collective opinion-formation will be democratically defective. It is precisely the debate in the background culture that generates the public opinion and collective preferences that are reflected in the decisions of the public political forum. Because these two phases of deliberation are inextricably tied - normatively and empirically - legitimate outcomes require that both be open, fair, and equally inclusive of all citizens. No matter how fair, inclusive, and neutral deliberation may be in the public political forum, outcomes will not be democratic if the preceding deliberation in the background culture is not also sufficiently open and fair.

By ignoring deliberation in the background culture because it is not tied to a formal decision-making body, and therefore does not immediately result in coercively enforced or "binding" decisions, the narrow understanding of public reason undermines the legitimacy of decisions reached in the public political forum. In light of the connection between deliberation in the background culture and the public political forum, I conclude that the narrow interpretation of public reason fails. We cannot limit our concern for fair procedures of deliberation to the public political forum. The narrow view of public reason does not go far enough in ensuring fair decision-making procedures in a pluralistic society.

Rawls contends that citizens have a moral duty of civility when deliberating across difference in the public political forum. This duty - again, realized through the idea of public reason - helps support legitimate decisions that respect differences among citizens. Having rejected the narrow view of public reason, we must consider whether the expansive view of 
public reason can ensure civility and a corresponding respect for differences in the background culture. I argue that it cannot.

The procedural guarantee of fair and inclusive deliberation that Rawls employs for the public political forum - namely the idea of public reason - cannot be applied to the background culture. ${ }^{8}$ Although I have shown the insufficiency of a narrow application of public reason to the public political forum, simply extending the limits of public reason to the background culture is not an effective solution.

The diffuse and informal nature of deliberation in the background culture makes it unaccommodating to the kinds of procedural guarantees of equal consideration and fairness that Rawls proposes for formal deliberation in the public political forum. Rawls himself acknowledges that, for practical reasons, the idea of public reason cannot be applied to the background culture. The background culture of a pluralistic democratic society is not "guided by one central idea or principle" $(1997,443)$. As a result, there is no shared set of reasons or standards toward which citizens could appeal when debating in this setting.

Given the inapplicability of the idea of public reason to deliberation in the background culture, it becomes clear that Rawls lacks the theoretical resources to account for fair and open deliberation across difference in that setting. In other words, even the expansive view of public reason cannot answer the question of how to ensure reciprocity and civility in the background culture where shared public reasons are not available.

\footnotetext{
${ }^{8}$ Again, I am tabling the question of the merit of the idea of public reason in the public political forum. I am engaging with Rawls on his own terms and picking up where his conversation leaves off.
} 
Given the inherent and necessary connection of the two stages of deliberation, the duty of civility - or a comparable guarantee of equal consideration across differences - must apply as much to the background culture as it does to the public political forum. The narrow understanding of public reason fails to ensure the legitimate and fair decision-making procedures that Rawls thinks it will. But the openness and reciprocity that is required for deliberation in the background culture to be inclusive of all perspectives cannot be guaranteed simply through formal procedural design. As a result, we need something other than procedural norms and rules to ensure fair and democratic outcomes of deliberation.

To ensure the democratic quality of deliberation in the background culture, I propose a shift in focus from procedure to the disposition or qualities of participants that can be understood as procedural preconditions. This shift will deepen our understanding of the necessary conditions of democratic deliberation. Without dialogically open citizens, informal deliberation in the background culture will not be truly inclusive of all perspectives. This is problematic insofar as public opinion is formed first in the background culture before being implemented into coercive laws, justified by public reason in the public political forum.

I show in the following sections that Habermas (1.2.ii) and Young (1.2.iii) depart from Rawls by offering accounts of democratic legitimacy that take seriously deliberation in the "background culture," or "public sphere." With their respective models of deliberative democracy both Habermas and Young give "more prominence to processes of discussion and citizen involvement in the associations of civil society than do most theories of deliberation" (Young 2001, 46). Their theories mark a stark departure from Rawls's model of deliberation which focuses primarily on the public political forum. 
By explicitly incorporating diffuse and informal deliberation into their theories of democratic deliberation, Habermas and Young both offer a more complete view of the processes of legitimate democratic decisions. In their expansive views of deliberation, Habermas and Young consider what - using Rawls's language - the "duty of civility" might require of citizens engaging in deliberation in the background culture. As I show below, however, Habermas and Young still suffer from a problematic assumption of openness among citizens.

\section{2.ii Habermas}

Compared to Rawls, Habermas pays much more attention to the question of deliberation in the background culture, or what he calls the "informal public sphere" $(1996,308)$. He explains that deliberative politics "lives off the interplay between democratically institutionalized willformation and informal opinion-formation" $(1996,308)$. His attention to the quality of deliberation that takes place at an informal level marks an improvement over Rawls's exclusive focus on the formal public forum. Yet Habermas still fails to attend to the question of ensuring that this informal deliberation will be sufficiently free and equal. Similarly deficient in "dialogical openness," Habermas lacks the theoretical resources for comprehending the kind of communicative generosity and receptivity that his model of discursive democracy requires.

Habermas understands deliberative democracy as operating along two tracks: the informal communication dispersed across public spheres, and the formal deliberation that occurs in official decision-making bodies like Parliament and Congress. The second track provides an institutional focus for the broader "subjectless" communication of the first track. First, communicative power is generated in informal deliberations taking place in the public sphere. This communicative power is then transformed into administrative power $(1994,8)$. It is the 
realization of this process that legitimizes coercive political decisions and actions. In fact, Habermas explains that "the democratic procedure can lead to a rational will-formation only insofar as organized opinion-formation, which leads to accountable decisions within government bodies, remains permeable to the free-floating values, issues, contributions, and arguments of a surrounding political communication that, as such, cannot be organized as a whole" $(1988,485)$. Decisions are legitimate insofar as they are reflective of and influenced by public discourse.

Habermas does well to focus on the complete process of collective opinion-formation that occurs before will-formation and collective action. But as I will show, the theory of communicative action on which he relies falls short in explaining the conditions for a truly discursive communicative processes.

The transformation of "communicative power" into "administrative power" is not sufficient for ensuring successful deliberation. An adequate theory of deliberation must secure adequate sources for dialogical openness in order to explain how communicative power is generated. The legitimacy of formal decisions made in the public forum depends on the discursive quality of the informal deliberative processes that generate communicative power in the first place $(1996,448)$.

Assumption of Openness in Theory of Communicative Action

The power of Habermas's discourse theory of democracy is rooted in his theory of communicative action. According to this theory, citizens are expected to present, challenge, and defend various validity claims to one another in an attempt to reach mutual understanding. "Insofar as actors wish to coordinate their action through understanding rather than force or manipulation, they implicitly take on the burden of redeeming claims they raise to others 
regarding the truth of what they say, its normative rightness, and its sincerity" (White 1995,7). The understanding achieved through language allows for coordination of actions based on consensus and not manipulation or coercion.

Communicative action "depends on the use of language oriented to mutual understanding. This use of language functions in such a way that the participants either agree on the validity claimed for their speech acts or identify points of disagreement, which they conjointly take into consideration in the course of further interaction" $(1996,18)$. With the presence of disagreement, ongoing communicative action is interrupted and discourse begins. At this point, language works to mediate a disagreement only if participants adopt the "performative attitude of a speaker who wants to 'reach understanding' with a second person about something in the world" $(1996,18)$. This performative attitude replaces the objectivating attitude, which is oriented to personal success. But how does this transition to the performative attitude take place?

It is the reciprocal nature of communication that allows for consensus and mutual understanding to be achieved. As opposed to strategic action, which aims at convincing (or deceiving) someone to participate in one's own predetermined end, communicative action does not necessarily presuppose the end towards which discourse will ultimately aim. The opinions and positions that are raised in communication are susceptible to the consideration and critique of others, which may ultimately lead to consensus (1994 I, 136). Habermas specifies that "the binding energies of language can be mobilized to coordinate action plans only if the participants suspend the objectivating attitude of an observer, along with the immediate orientation to personal success, in favor of the performative attitude of a speaker who wants to reach an understanding with a second person about something in the world" $(1996,18)$. 
Habermas does not explain the origin of the wish for understanding that sustains communicative action. He appears to assume that this orientation is an automatic or an inherent part of language itself. As he explains in his Theory of Communicative Action, "reaching understanding is the inherent telos of human speech" (1994 I, 287). Mutual understanding is the natural end of our communicative encounters with others.

While I am generally sympathetic to Habermas's model, especially its inclusion of informal deliberative encounters, I argue that the assumption of reciprocity and openness to others' opinions requires a corresponding theoretical exploration of their source. In a political context where groups and individuals are potentially vying for scarce resources or conflicting policies or laws, the assumption that citizens would adopt such a performative attitude needs justification. As Romand Coles explains, for the most part, Habermas resists “ontological harmony claims" $(1997,15)$. He does not assume that consensus is a natural or automatic part of the human condition. For Habermas, agreement is not inherent to the human condition, but an orientation toward agreement is. Coles sums up Habermas's position well: “because our existence and coexistence are deeply communicative, we are ontologically and normatively characterized not by de facto agreement but by a mutual lived commitment (agreement) to coexist through efforts to agree" (Coles 1997, 18). Although Habermas's assumptions regarding mutual understanding and consensus are much weaker than critics often charge, he still has a yet unjustified assumption regarding our general "orientation" toward mutual understanding.

The assumption of reciprocity and the maintenance of a performative attitude, which may be valid and empirically justified in ongoing communicative action where there is no perceivable disagreement, does not automatically obtain in discourse. In Habermas's model, discourse only 
begins once ongoing communicative action has been disrupted by a disagreement among citizens. The presence of disagreement makes his assumption of an orientation toward mutual understanding even less convincing. Why, in the presence of disagreement, when discourse has interrupted ongoing communicative action, would citizens maintain the performative attitude instead of switching to the objectivating attitude of strategic action. Habermas asserts that this performative attitude will continue as a function of our capacity for language.

Of course, the empirical question of why or how people adopt the performative attitude may be ancillary to Habermas's theory of the normative conditions for legitimate decisions. A full explanation of how this is brought about may not be required for his theory of democracy. Habermas might insist that he is only interested in making the normative claim that decisions reached between citizens who fail to adopt the performative attitude are not democratically legitimate. But given that Habermas's model of deliberation explicitly relies on his account of how language works, fleshing out the transformation from the objectivating to the performative attitude is crucial.

When Habermas writes that "the telos of language is mutual understanding," he assumes that openness or reciprocity is an automatic component of speech. He famously claims that the hallmark of modernity is our ability to provide reasons for our opinions and our willingness to be swayed only by "the forceless force of the better argument." But being swayed by the forceless force of the better argument is not a given, especially when deliberation occurs among citizens with different experiences, values, interests, comprehensive doctrines, etc. I maintain that citizens must be primed if their wills and opinions are to be moved through the forceless force of reason. 


\section{2.iii Young}

Departing from Rawls, but following Habermas, Young "advocate[s] a 'decentred' conception of politics and society...Society is bigger than politics and outruns political institutions, and thus democratic politics must be thought of as taking place within the context of large and complex social processes the whole of which cannot come into view, let alone under decision-making control" (Young 2000, 46). In such a decentered model of deliberative democracy, "the democratic process cannot be identified with one institution or set of institutions...Rather, the processes of communication that give normative and rational meaning to democracy occur as flows and exchanges among various social sectors not brought together under a unifying principle" (Young 2000, 46).

Before I explain how Young's procedural model of democratic deliberation insufficiently addresses the concerns of difference democrats raised in the first section, I should explain the various - and seemingly contradictory - ways that I cite her work in this chapter. In the beginning of this chapter, I grouped Young with "difference democrats" who take issue with the ways in which extant theories of deliberation fail to accommodate difference in democracy. Now in this section, I turn Young's criticisms against her own model of communicative democracy. Despite this seeming contradiction, I still would place Young squarely in the camp of "difference democrats." And yet she remains committed to communication as the best means to bring about just political decisions. Although a critic of some models of deliberative democracy, Young does not wholly reject deliberation as do agonistic democrats who share her concern for difference.

While aware of some of the exclusionary tendencies of deliberation, Young advocates a model of the democratic process that "retains deliberative democracy's account both of 
communicative orientation towards normative reason and of the transformation of private, selfregarding desire into public appeals to justice" $(2000,51)$. Despite her commitment to communication as the best means to bring about just political decisions, I would argue that Young is as worried about threats to justice that exist in the presence of deliberation as she is to threats to justice in its absence. Accordingly, she raises important concerns regarding the inhospitality of traditional deliberative processes of democracy to the question of difference.

Like Rawls and Habermas, Young provides a robust account of how democratic deliberation can bring about just outcomes within large, pluralistic societies. She differs from them, however, in what she identifies as the major threat to this outcome. As I show above, Rawls focuses on the threat that non-public deliberation poses to achieving just outcomes. He wants to make sure that people are not forced to live by laws that are motivated by comprehensive doctrines to which they do not subscribe. By maximizing the public nature of our deliberation, Rawls hopes to maximize the legitimacy of decisions. Habermas, on the other hand, is most concerned with the free flow of communicative power and its transformation into administrative power. Laws are legitimate only if they have been shaped by the informal and decentralized communication of citizens. Habermas attends to maximizing the permeability of sites of administrative power so as to ensure the influence of communicative power. We are always already oriented towards reaching understanding with one another through language. Habermas's main priority, then, is ensuring that institutions are open to the input of public spheres and therefore that laws reflect the inter-subjectively rational will of the people.

For Rawls and Habermas, legitimacy is all but guaranteed through the adoption of their procedures. For Young, however, the hard work of ensuring legitimacy and justice lingers even 
after these procedures have been adopted. Young helps us see the ways that even communicatively achieved decisions can be undermined by the very procedures of deliberation meant to ensure their legitimacy. As a critic, she points to the ways in which even the ideal procedures of Rawls and Habermas produce non-ideal outcomes.

One of Young's most important contributions to democratic theory has been her challenge to the kinds of communication that have traditionally been admitted into deliberation. She argues that the inclusivity and openness of deliberation are undermined by Rawls's and Habermas's preference for rational argumentation. She rejects the assumption that argumentation will be persuasive only by the "forceless force" of being superior. Young rightly points out that the sorts of restrictions on the kinds of speech permitted in democratic discourse are not "culturally neutral and universal," pointing to the "way that power sometimes enters speech itself" $(1997,63)$. Concerned about the ways in which power can operate through language in invisible or undetectable ways, Young aims at reducing some of this power by pluralizing the means and modes of communication.

To make deliberation more inclusive of differences, Young proposes admitting additional forms of communication including greeting, rhetoric, and story-telling. For Young, restricting deliberation to argumentative speech has the potential to generate damaging forms of hierarchy and power dynamics. Taking her aim of inclusiveness even further, Young argues that "Disorderly, disruptive, annoying, or distracting forms of communication are often necessary or effective elements in such efforts to engage others in debate over issues and outcomes" (2000, 50). To make deliberation more open and inclusive of all voices and perspectives, Young loosens 
the restrictions placed on the types of reasons and the forms of communication that can be used in deliberation.

Young productively identifies new ways to design deliberative institutions so that relevant voices will be included. "Demonstration and protest, the use of emotionally charged language and symbols, publicly ridiculing or mocking exclusive or dismissive behavior of others, are sometimes appropriate and effective ways of getting attention for issues of legitimate public concern" (Young 2000, 66). Permitting disorderly, disruptive, or annoying forms of speech necessarily makes the procedures of communication more open and more broadly inclusive. But the citizens who are hearing this speech are not any more likely to engage with these forms of communication than they are with the rational and orderly argumentation of Rawls or Habermas. Despite these important improvements to the inclusiveness and openness of deliberative processes, Young seems to conflate the adoption of more open procedures with more receptivity or openness on the part of citizens. The problem of dialogical closure, if not procedural closure, remains.

Missing from Young's discussion is an account of how citizens might be drawn to listen to each other and actually consider alternative opinions especially the newly included forms of communication. Either Young does not recognize the importance of priming citizens to be receptive to these and other more traditionally accepted forms of political speech, or she assumes that citizens will simply be more likely to engage these rhetorical, disruptive, and affective modes of communication. While the latter position may certainly be true, it is at least worth exploring why these forms of communication would have more success in drawing some citizens to engage perspectives that they would otherwise ignore. 
Young is right to discuss the ways in which restrictive or closed procedural design can undermine the very goals of inclusion and fairness. But when it comes to the disposition required of citizens, she too includes it merely as an assumption or initial premise of democratic deliberation. Young, like Habermas, assumes a high level of receptivity and openness on the part of citizen-listeners. For example, Young lists "reasonableness" as a prerequisite for democratic deliberation. Importantly, Young redefines "reasonableness," distinguishing her concept from a narrow view of "reasonableness, or civility," often associated with Habermas, and which can be "used to locate some people as temperate and to label as 'extreme' others who use more demonstrative and disruptive means" (Young 2000, 47). Young shifts the focus from reasonable speech to "reasonableness" as a willingness to engage in the first place.

Her expansive definition of "reasonableness" is explained as a willingness "to listen to others, treat them with respect, make an effort to understand them by asking questions, and not judge them too quickly" (Young 2000, 25). Young's new and improved statement of the condition of reasonableness gives us an idea of the kinds of attitudes citizens must adopt if their communication is to result in legitimate democratic decisions.

Given the lengths that Young goes to ensure that formal and informal deliberation will be inclusive of all perspective and diverse forms communication, her assumption of openness or “reasonableness" among citizens engaging informally in the public sphere seems perfunctory. The open disposition among citizens that Young assumes as an initial premise of her model of democratic deliberation is not automatic and instead must be cultivated. In the final section of this chapter, I explore this disposition in greater detail. What would an attentive listener or a dialogically open citizen look like? I argue that if citizens are to meet Young's expectation of 
reasonableness, they must be drawn to show a significant level of humility, receptivity, and generosity in their interactions with others.

Although Habermas and Young build the need for openness into their models - through their respective discussions of the "performative attitude" and "reasonableness" - neither adequately theorize the source or origin of this orientation. Although the omission of the nature and sources of dialogical openness is problematic for both Habermas's and Young's account of democratic deliberation, their work is instructive in pointing to the need for it in the first place. Therefore, I consider the argument that follows to complement and grow organically out of their discussion of deliberation in the background culture.

\subsection{Dialogical Openness}

So far, I have highlighted the ways in which Rawls, Habermas, and Young do not adequately attend to the nature and sources of dialogical openness among citizens in public discourse. Rawls is unable to address the issue of dialogical openness because of his inattention to deliberation in the background culture. Habermas, too, turns a blind eye to the need for dialogical openness in deliberation insofar as he assumes that mutual understanding is the telos of language. Lastly, Young focuses on making deliberation more open, but assumes that more inclusive procedures will bring about more open citizens.

This inattention to dialogical openness prevents extant models of deliberation from adequately addressing challenges regarding democracy and difference. Only a model of deliberation that provides adequate sources of dialogical openness will be able to accommodate deep differences - ensuring that they are considered rather than simply permitted in discourse. In their contention that "reciprocal reason giving is going to form an effective or stable basis for the 
moral validity of agreements in a pluralistic society," Rawls's, Habermas's, and Young's models each, in their own way, rely not only on a willingness to justify one's own position to others but also "a prior willingness to actively engage, listen to, and learn from diverse others" (Button 2005, 860). All three fail to consider or explicitly articulate the disposition on the part of citizens that is implicitly working in the background of their theories of deliberation. In this section, I outline the disposition required of citizens engaging in deliberation, before discussing the related question of how this disposition might be cultivated or brought about in Chapters 2 through 4 .

Outside of the context of procedural theories of democracy, the question of disposition and ethical orientation of citizens has been a topic of renewed debate. For example, agonistic democrats, with their eyes already trained on the deep differences between people, have theorized more extensively about the kind of disposition that would allow deeply divided citizens to engage agonistically rather than antagonistically. As Stephen K. White explains, the most credible agonistic democrats are acutely aware of "the cognitive and affective need to dampen the initial wariness and certainty that we are likely to carry in our engagement with those whom we all too easily size up as radically other to us" (2009, 31). William Connolly and Stephen White have both dedicated much attention to theorizing a concept of "presumptive generosity" in an attempt to explain one way that citizens can engage across deep constitutive differences. The idea I present here, regarding the nature of dialogical openness draws on their work; however, I approach the question of democratic dispositions from a more explicitly deliberative angle. Rather than looking skeptically at the deliberative goal of mutual understanding and agreement, as Connolly does, I argue that an orientation toward consensus, though not an inherent or automatic human capacity, is still possible and worth pursuing. 
The procedural justice to which deliberative democrats subscribe actually requires that we look beyond or, perhaps more accurately, before procedure and examine the kind of citizen who will engage openly in deliberation. By drawing attention to the importance of dialogical openness, which I identify as an affective disposition on the part of citizens, I am following the more recent tradition of democratic theorists who make room for the role of affect in democracy (Krause 2008, Morrell 2010, Nussbaum 1995, Abizadeh 2007, White 2009). The affective component of deliberation, in my understanding, works as a complement to the rational argumentation that takes place among citizens. As Arash Abizadeh explains, Habermas actually weakens his own model and opens it up for criticism by constructing "the notion of discursive rationality in contrast to, and in abstraction from, the rhetorical and affective components of language use" $(2007,445)$. Until we "reject the false dichotomy between reason and passion in both political theory and American public life" we undercut "our ability to advance the cause of justice" (Krause 2008, 7). By encouraging the incorporation of affective elements - specifically the affective disposition of dialogical openness - I hope to weaken the sharp opposition between rationality and affect, reason and passion.

I ask: if people are to be swayed only by "the forceless force of the better argument" and if they are to engage with the claims and preferences of their fellow citizens, what kind of disposition is required? What attitudes must citizens have towards each other in order for their deliberative encounters to be fair and equal? What would an empowered listener or a dialogically open citizen look like?

It does not represent a threshold that is to be met, nor is it a capacity that can be turned on or off. Dialogical openness creates the metaphorical space where citizens come to engage with 
one another. Instead of imagining deliberation as being a door that can be open or closed to participants - I propose thinking of openness in spatial terms. Turning away from the idea of openness as procedural "access," we expand the concerns of deliberative democrats beyond the question of voice and speech. As I have argued throughout this chapter, procedural guarantees of openness can ensure only open "access" to deliberative forums, not the corresponding engagement and consideration that makes the access meaningful in the first place. Struggles for inclusion and engagement continue, and maybe only truly begin, once access has been achieved. Only with this more expansive view of openness can we attend to the goal of equal consideration, which is at the heart of the promise of democracy. Given the inevitability of difference and disagreement, legitimate decisions can only be made if participants in discourse have a particular disposition - one of humility, receptivity, and generosity characterized by a deep openness in dialogue.

Humility is what allows for the conversation to take place at all. Drawing on the “epistemic and social-relational dimensions of humility," Mark Button defines democratic humility as "a cultivated sensitivity toward the incompleteness and contingency of both one's personal moral powers and commitments, and of the particular forms, laws, and institutions that structure one's political and social life with others" $(2005,841)$. If we do not exercise humility when engaging with our fellow citizens, then deliberation is not likely to get off the ground. Without humility - in relation to our own knowledge, ideas, and especially our understanding of the question and stakes involved - we have no need to engage with one another. If I am wholly confident in my own understanding - even if not mutual understanding - then decisions can be decided monologically. Button describes humility as "a window through which we allow that 
which is outside of the self or group to enter in and work upon us, at least for a time" (2005, 851). This understanding of democratic humility is "supportive of cognitive/affective openness, a spirit of attentiveness and active listening" insofar as it demonstrates the value for engagement in the first place (Button 2005, 851).

After the initial exercise of humility sets the stage for engagement, providing an opportunity, receptivity is what provides the space for engagement to occur, as if we were receiving someone into our home. In our receptivity, we acknowledge the relevance and contribution of someone else's perspective. We show curiosity and a desire to hear what they have to say.

As I discuss further in Chapter 3, through the exercise of generosity, citizens show patience and allow the other to express herself before jumping to any conclusion. Generosity can only be sincere and not patronizing with the initial exercise of humility, which ensures that the engagement has value and is not akin to simply "going through the motions."

Dialogical openness is an active ethos that must be cultivated among citizens if their deliberation is going to be as inclusive and solicitous as proponents of deliberation assume it will be. Dialogical openness is a state or disposition of engagement, it is not itself a motivation to engage. Furthermore, although I refer to it as a prerequisite for successful deliberation, this orientation towards the other does not always occur before political engagement begins. And it is not necessarily something that citizens develop on their own. This openness is perhaps just as likely to be demanded or extracted by a minority group as it is to be freely offered by a majority. In other words, while this openness can be cultivated internally, others also demand it. For example, we can see a demand for openness in actions taken by the Occupy Wall Street 
movement. During the height of this movement, activists forcibly occupied space that would have otherwise been closed off to them.

As I discuss in Chapter 3, citizens' encounters with those who are most different from

them may be the best way to draw them in the direction of greater open-mindedness. We should be attentive to what dialogical openness looks like, even if we cannot rely on citizens cultivating it on their own. Articulating the virtues, capacities, or dispositions of engaged citizen-listeners marks a contribution to the existing literature, much of which assumes that they either are inherent to citizens or can be replaced by elements of procedural design such as the idea of public reason.

\subsection{Realist Critics of Deliberation}

Some critics of democratic deliberation might take issue with my claim that dialogical openness will meaningfully improve the inclusivity of difference in deliberation. For these realist critics, communication is not the best way to address problems of power and exclusion operating in politics. Despite looking beyond deliberative procedures, these critics maintain a decidedly procedural focus, looking to procedures of decision-making, specifically distributing decisionmaking power and authority fairly (though perhaps not equally in an absolute sense) among citizens. Instead of focusing on making communication more open and equal so as to achieve unforced consensus or agreement, these theorists favor a focus on (re)distributing real political power across individuals and groups. Only such a distribution of power can ensure all relevant parties' interests and concerns are represented. For example, Ian Shapiro argues that rather than focus on procedures of deliberation, the problem of domination is better addressed by giving rights of delay or veto to those whose basic interests are at stake $(2003,48)$. Similarly, even 
Young points to the importance of these extra-communicative efforts, suggesting that relevant minorities be given veto power over policies "that fundamentally and uniquely affect members of their group" (1990, 189).

The problems of an exclusionary public sphere are certainly mitigated when procedures of decision-making protect the influence of otherwise marginalized groups. For example, giving historically underrepresented groups veto power guarantees that they are not ignored out of hand. Furthermore, Bohman rightly points out that "if power over decisions is widely dispersed in institutions...excluding groups from deliberation is more difficult to accomplish" (Bohman 1996, 148). If we have decision-making procedures that 'nudge' citizens to confer with historically marginalized groups, do we need dialogical openness? If certain groups have veto power over policy domains, does it matter if people actually listen to each other? Why do we need deliberation in the first place?

Although certain protections should be institutionalized to ensure the special representation of otherwise socially excluded groups, we should also continue to seek communicatively mediated agreement that is inclusive of all perspectives. "Power shifts may bring improvement, but only accidentally. Changes of mind are responsive to reasons that at least direct our attention toward improvement. When majorities are obligated to offer reasons to dissenting minorities, they expose their position to criticism and give minorities their most effective and fairest chance of persuading majorities of the justice of their position (Guttman and Thompson 1996, 44). Although an imperfect tool, language still provides individuals and groups with significant power. 
I would be more sympathetic to these realist critics if all avenues of procedural redesign and preconditions had been exhausted. But given that there is still so much terrain with which to address issues of dialogical closure and failures to communicate, I find their rejection of democratic deliberation to be too hasty. Although I commend political theorists who seek to study economic, political, and social inequality, the scope of useful and productive deliberative theory is broader than they allow. My project operates according to the view that just decisionmaking is impeded not only by the presence of limiting conditions, such as inequality, but also by the absence of certain facilitating factors. Although we will struggle to resolve many of the intractable limiting conditions, such as economic inequality and cultural hegemony, we can continue to make improvements by introducing these facilitating factors to the process of democratic decision-making.

\subsection{Conclusion}

As I have shown, Rawls, Habermas, and Young all propose models of democratic deliberation that are deficient in theoretical sources of "dialogical openness." By relying on formal procedures to guarantee open deliberation among citizens, Rawls is unable to account for the openness and inclusiveness of informal, yet politically significant, deliberations occurring among citizens in the background culture. Through his view of language, Habermas merely assumes the reciprocity that deliberation requires. And Young, though concerned with many of the same issues as myself, assumes that more open and inclusive procedures will lead to more openness on the part of citizens.

Moving beyond the question of procedures, this dissertation is interested in the question of the disposition required of citizens engaging in deliberation. More specifically, my concern is 
pushing these attitudes in the direction of greater open-mindedness. I do not expect to find one solution to the problem of dialogical closure. Attention to this problem, however, is an important first step in correcting it. And my hope is that, through our examination of the problem of dialogical closure, we can begin to address some of the concerns that critics of deliberative democracy have addressed since the deliberative turn. 


\section{Chapter 2. Beyond Empathy: Strategies and Ideals of Democratic Deliberation}

As the deliberative approach to democracy has emerged into prominence over the last quarter century, scholars have increasingly attended to the capacities citizens must have if their deliberation is to enhance democratic legitimacy in the ways its advocates envision. One capacity often cited is empathy. The recent appeals to empathy by political theorists, typified by the work of Sharon Krause and Michael Morrell, but also that of Michael Frazer, Robert Goodin, Martha Nussbaum, and others, have grown out of a new emphasis on the value of affect, emotion, and passion in democratic discourse.

As James Bohman explains, theories of democratic deliberation "share the common demand that democracy is the rule by citizens of their common affairs through the public use of reason" (my emphasis; 2004, 23-24). The recent turn to empathy has developed as a reaction to what was considered a narrow or singular understanding of reason in theories of deliberation, especially those of Rawls and Habermas (Marcus 2013).

Although the disputes over reason and emotion in democratic deliberation are far from resolved, members of both the affective and rationalist camps agree that empathy sustains democratic legitimacy by promoting impartiality and inclusiveness in political communication among citizens. ${ }^{9}$ Empathy is typically viewed as one of a few “"good' emotions," - compatible with rational discourse and reasoned argumentation (Marcus 2013, 131).

\footnotetext{
${ }^{9}$ Sympathy and empathy are often used to mean the same thing. Part of this confusion comes from the relatively recent appearance of "empathy" in the English language. "Empathy" was coined from the German word Einfühlung in the early $20^{\text {th }}$ century. The word simply did not exist when David Hume and Adam Smith were writing about imagined perspective taking and shared feelings, which they included under the concept of "sympathy." Sharon Krause, who refers exclusively to "sympathy" in Civil Passions, actually distinguishes between two uses of the word "sympathy" in the work of David Hume. As she and Michael Morrell admit, their preferred terms of sympathy and empathy, respectively, have a common meaning.
} 
In this chapter, I consider the value of empathy specifically within the context of democratic deliberation. Problematizing empathy in a novel way, I argue that empathy is too high a standard to pursue in democratic deliberation given the practical limits and barriers to successful perspective taking and empathic concern. Because of the natural variation in our ability to empathize and our tendencies to project our own views onto others, empathy cannot, in practice, generally be relied upon to play the central role in deliberation that its supporters expect it will. Furthermore, I show why simply aiming for more empathy is an unlikely answer to these limits of empathy in practice. ${ }^{10}$

Specifically, I argue that the empathy approach is flawed even as an ideal in democratic deliberation. Even if we are able to successfully imagine how another person thinks or feels, empathy would still tend to displace other, more effective ways of engaging the crucial deliberative concern of improving practices of democratic listening. Even when attempts at empathy succeed, they do not improve democratic deliberation. Advocates of the empathy

${ }^{10}$ Despite its wide acceptance among political theorists, especially deliberative democrats, two nascent critiques of empathy can be distilled from the broader empathy literature in moral philosophy and psychology. The few who have taken issue with empathy focus on its purported limits (Prinz 2011). First, feelings of empathy are said to be weak and easily overcome by other concerns. Jesse Prinz, for example, explains that empathy "does not motivate moral [or prosocial] behavior when there are significant costs $(2011,222)$. Second, empathy is said to bias our decisions and judgments. We give preferential treatment to those with whom we empathize. As some have pointed out, however, these concerns may actually be alleviated with more empathy. In other words, if empathy was stronger, it would not be as easily trumped by self-interested concerns. And if we experienced empathy more widely, and perhaps in relation to all people, we would have less reason to fear any unfair bias. As Robin West sums it up in her essay on empathy and jurisprudence, "the target of the anti-empathy argument is not empathy per se but selective empathy..." $(2013,247)$. In this chapter, I demonstrate why more empathy cannot be the solution to the problems of empathy addressed here. In the first section, I use empirical evidence to suggest that these limits cannot, in fact, be overcome. In the second section, I show why even successful attempts at empathy do not sustain deliberative practices. In other words, simply more empathy is not the solution. 
approach fail to see how an overemphasis on empathy can sometimes lead us to believe that a perspective has been understood and thus "included" when it has not really been. I will explain that contrary to our intuitions, and perhaps paradoxically, empathy does not promote democratic inclusion in the way its promoters think it will.

In Chapter 3, I introduce and develop an alternative to the empathic approach, what I call the difference approach. By focusing on differences instead of real or imagined commonalities, citizens become more prepared to face the challenges of effective deliberation.

\subsection{Empathic Turn in Deliberative Democracy}

The Oxford English Dictionary defines empathy as "the power of projecting one's personality into (and so fully comprehending) the object of contemplation." Students of politics, philosophy, law, and psychology have all offered their own definitions of empathy. But across these disciplines, empathy is understood as both a process and an outcome. ${ }^{11}$ Empathy-asprocess, which involves imagining another's perspective, is supposed to bring about empathy-asoutcome, of which there are two types - cognitive and affective. Cognitive empathy is the "awareness of another's feelings," whereas affective empathy is "feeling what another feels" (Hoffman 2011). Cognitive empathy allows us to understand another's perspective or feelings, even if we do not ultimately come to share them.

The procedural nature of empathy is obvious when tracing its relatively recent etymological roots. The origin of the word "empathy" can be traced to the German, Einfühlung, which means "feeling-into." According to Frazer, "Herder argues that we imaginatively place

\footnotetext{
${ }^{11}$ The value of empathy has sparked rich debate in moral philosophy, jurisprudence, and psychology as well as in democratic theory, which is the general topic of my dissertation. Unfortunately, I have found that much of these discussions are happening in isolation.
} 
ourselves into the place of others, 'feeling our way into' their experience of the world"' (154). Herder developed this term in regards to reading historical texts, where historians could only understand ancient peoples through a "process of self-projection" (Frazer, 155).

The process of self-projection, or imagined perspective-taking, is said to improve deliberative practices, by serving an important informational function. In Civil Passions (2008), Krause relates the deliberative ideal of impartiality to sympathy. "Impartiality, the ability to adopt a common point of view, involves abstracting from one's own self-interest but not by means of disengaged intellect. Instead, we achieve impartiality by sympathetically experiencing the sentiments of others" (Krause 2008, 73). According to Krause, judgments cannot be made without reference to passions. Therefore, impartiality does not require the exclusion of passion and sentiment, but instead requires the inclusion of the passions and sentiments of all relevant individuals. For Krause, sympathy is the basis for making judgments that are inclusive of all citizens' perspectives and sentiments.

Krause identifies two distinct, though related, meanings of sympathy in the work of David Hume, one cognitive the other affective. The primary meaning of sympathy for Hume is what Krause calls S1. S1 "is not itself a passion, hence not itself an affective state, but it communicates passions to us and stimulates similar passions in us" (Krause 2008, 80). As a cognitive faculty of the mind "with an informational function," S1 provides affective inputs for our consideration in deliberation (Krause 2008, 79). S1 allows us to know how another is feeling. The feelings of others do not need to be explicitly communicated to us, and instead are often imagined (Krause 2008, 85). Though primarily serving a cognitive and informational function, 
sympathy in this sense "enables us to resonate with the affective experiences of others, to be moved by the sentiments that others express" (Krause 2008, 80).

Krause refers to Hume's second sense of sympathy as S2. S2 "is itself an affective state, or a form of passion" $(2008,80)$. Sympathy in this sense involves caring for another person. Krause explains that S2 is the benevolence or pity that, quoting Hume, "makes me concern'd for the present sorrows of a stranger" $(2008,80)$.

Hume does not differentiate between S1 and S2, but Krause shows how the two senses of sympathy are different, though related. Deploying the cognitive faculty of sympathy (S1) can often lead to affective concern for others, or S2 (Krause 2008, 81). "Yet even where the effects of sympathy are too weak to motivate concern for the well-being of another, the sentiments conveyed still provide the grounds for judgment" (Krause 2008, 81). Even if exercising the cognitive faculty of sympathy (S1), or empathy-as-process, does not produce feelings of sympathy for others (S2), it is still an important contribution to the impartiality of moral judgment insofar as it introduces the sentiments of others as relevant inputs for deliberation. In other words, according to Krause, the value of empathy comes in large part from the empathic understanding that results from imagined perspective taking.

Although sympathy (S1) is "automatic" and "a natural feature of our moral psychology," it "is not naturally as extensive or as free from self-love as impartial judgment requires" (Krause 2008, 84). Krause argues that sympathy is an important, though limited, first step in knowing the sentiments of others so as to include them in deliberation. Sympathy makes our consideration more inclusive, helping us cultivate a truly generalized perspective. 
Building on Krause's endorsement of both the cognitive and affective side of sympathy, Michael Morrell's Empathy and Democracy (2010) offers additional reasons for incorporating what he calls empathy into democratic deliberation. Morrell argues that democratic legitimacy depends on "whether people empathized with their fellow interlocutors" $(2010,193)$. Both Krause and Morrell aim to recast "deliberative theory to allow for a cognitively and affectively mixed process of sympathy or empathy in order to inform the judgments people make in deliberation" (Morrell 2010, 194).

Besides Krause and Morrell, other theorists have argued that concepts similar to Krause's sympathy and Morrell's empathy make important contributions to democracy. For example, Richard Rorty (1989) offers "imaginative identification" as the basis of solidarity, while Goodin (2003) discusses the need for "empathetic imagining" as an aspect of "deliberation within." Other theorists appeal to the assumed benefit of empathy. Diana Boros (2012) and Martha Nussbaum (1995), for example, both write on the value of art for democratic politics for its ability to generate feelings of empathy. Boros writes that "a feeling of universal human community among individuals," which she equates with empathy, "is not only valid and in need of renewal, but is also a key to solving the lack of participation in American public and political life" $(2012,111)$.

Taken together, I argue, these approaches to deliberation constitute an "empathic approach." The central claim of this approach is that without empathy - or the "affectivecognitive communication of sentiments between persons that transpires through perspectivetaking” - "deliberation cannot provide a basis for legitimate, justified democratic decisionmaking that truly takes all into consideration" (Krause 2010, 83; Morrell 2010, 194). In order to 
keep discussion here manageable, I will engage primarily with Morrell and Krause, taking their work to be a proxy for proponents of empathy in general. Although discussions of empathy's contribution to democracy are appearing with greater frequency, Krause and Morrell provide the most in-depth and detailed accounts of its contribution to democratic deliberation.

Here, I focus on the presumed value of this imagined perspective-taking for the theory and practice of democratic deliberation. I will argue that this process is not effective at achieving either affective or cognitive empathy. Furthermore, even when successful, this process and its outcomes do not improve democratic deliberation.

\subsection{Three Practical Limits to Empathy}

Michael Morrell offers us what he calls the "process model of empathy." The process includes "antecedents" (including biological capacity and observer/target similarity) that interact with the "mechanisms of empathy," especially perspective taking, which Morrell claims is the most relevant to theories of deliberation. Together the antecedents and mechanism of perspective taking lead to both affective and cognitive outcomes.

Imaginatively taking the perspective of others may lead to feelings of empathic concern for others. But even if this exercise does not result in greater feelings of empathic concern, perspective taking still produces the cognitive outcome of empathic understanding. Empathic understanding allows me to know and understand the sentiments of others even if I do not ultimately come to share those feelings.

Morrell's inclusion of empathic understanding mirrors Krause's claim that S1 is valuable in its informational capacity even when it does not lead to S2 or feelings of concern for others. Both Morrell and Krause argue that empathy-as-process has an informational value even when it 
does not lead to feelings of concern for others. At the most basic level, imaginative role taking communicates the sentiments and perspectives of others to us, so that they may be included in our deliberation.

In this section, I argue that even the more modest outcome of empathic understanding is difficult to attain. Not only does perspective taking rarely lead us to affective empathy (empathic concern for others or feelings of sympathy), but our ability to actually understand the feelings of others is limited. That is to say, cognitive empathy - our ability to imaginatively encounter the feelings of others - does not give us access to the actual feelings and sentiments of our fellow citizens.

I question the empirical claim that empathy-as-outcome will in fact result from empathyas-process. Because of the difficulty of achieving either the cognitive or affective outcomes through imagined perspective taking, the empathy approach is not a realistic strategy for making democratic discourse more inclusive.

The expectation of successful outcomes from the process of empathy is unwarranted because of three important limits to empathy in practice. First, as Morrell acknowledges, people's empathic abilities vary widely. Some people are innately better than others at imaginatively taking the perspective of others or feeling empathic concern. Although Morrell mentions the inconsistency of empathy across individuals, he fails to adequately address the implications for deliberation. Second, even those individuals who are able and willing to engage in perspective taking tend to unconsciously project their own views onto others. Importantly, I draw on empirical research here that suggests that projection is how we engage in perspective taking rather than a sign of our failing to do so. Biasing our assessment of how another feels, 
projection limits the contribution that the process of empathy can have in making deliberation more inclusive. Third, all else equal, we tend to feel empathic concern and gain empathic understanding for people who are similar to us and less with people who are different. Thus the outcomes of Morrell's model are realized selectively and unevenly depending on the relationship of the subject to the target. Morrell cites empirical research that confirms empathy is hardest to achieve for "outgroups" and those who are most different from ourselves, but he does not address how these challenges can be realistically overcome (Davis 1994; Mutz 2002). Given these challenges to gaining empathic concern or understanding across difference, it seems unlikely that the problem of exclusion in deliberation can be overcome through empathy.

\section{2.i Limited Predispositions}

In this section I argue that because the antecedents to and the outcomes of the empathic processes vary so widely among individuals, the practical contribution of the empathic approach is of limited value. The process of empathy is not universally experienced, and so relying too heavily on it can stall political efforts to promote justice.

Morrell claims that "people with greater empathic dispositions are more likely to tolerate, be open to the views of, and not stigmatize those with whom they disagree" $(2007,386)$. Though democratically significant for the reasons Morrell lists, our predispositions for empathy especially empathic concern, but also our ability and willingness to engage in perspective taking - are not universally shared, nor are they easily improved. Empirical evidence shows that the success of the process model of empathy is determined in large part by the natural variation in empathic predispositions among individuals (which is assumed to be 'given' in Morrell's model). 
Morrell attempts to address concerns about the limits and variability of empathy by appropriating these concerns as another reason "for us to want to educate democratic citizens to have greater empathic predispositions for perspective taking and empathic concern" $(2007,398)$. But there are two weaknesses to Morrell's assumption that civic education can solve the problems of limited empathic predispositions.

First, Morrell does not specify what kinds of efforts might bring about these improved predispositions. Even in an article entitled "Empathy and Democratic Education" (2007), Morrell avoids any discussion of what empathy training would look like. His stated aim is simply to argue that “increasing citizens' empathic predispositions should be an important part of democratic education" $(2007,381)$. We can infer from his discussion of the experimental literature, however, that Morrell does not believe instructing subjects "to think about the target's situation or point of view" is sufficient or even approximates a truly empathic predisposition (2007, 398). Morrell's discussion of empathy education remains vague, as he avoids articulating either a specific strategy (educative or otherwise) or even a single example of how we might bring about more empathic predispositions. Morrell's point about education is that it should be aimed at increasing citizens' empathic predispositions; he does not speak to how we might do that.

The vague appeal to improving empathic predispositions through education is even more problematic given the empirical evidence Morrell himself cites suggesting that our empathic predispositions are largely inherited rather than determined by environmental factors, including education. Psychologist Mark Davis shows that "genetic factors seem to make a substantial contribution to individual differences in affective empathy," especially to differences in feeling 
empathic concern $(1994,65) .{ }^{12}$ Though Davis cautions that the specific heritability estimates may be somewhat inflated, he notes that for empathic concern these estimates hover around 70 percent compared to the 40 percent heritability estimate typically found for other personality traits, including extraversion or agreeableness $(1994,65)$. The extent to which empathic predispositions are inherited or "given" by genetic factors make them less amenable to intervention or improvement. Given our varying empathic capacities, subjecting deliberation to empathy becomes problematic when we consider debates over same-sex marriage or immigration. If empathy were to drive these debates then political communication would be likely to break down or stall when met with individuals' innate imaginative inflexibility or deficiencies in compassion.

Although I would favor efforts to design civic education programs to help bring about greater inclusiveness of and curiosity toward alterity, I am not convinced that such education programs should be directed toward improving empathy. Given the limits of both our empathic predispositions and the available means to change these predispositions, we should be skeptical of the contribution empathy can make in deliberation. While education may be able to improve these predispositions at the margins, Morrell himself acknowledges that these dispositions are in large part given. For example, Morrell excludes these predispositions as exogenous or antecedent to his “process model of empathy." Furthermore, Morrell's vague account of how we might improve our empathic predispositions through "education" does not take into account the evidence suggesting that they may be less susceptible to environmental cues and influences than

\footnotetext{
${ }^{12}$ Morrell's process model of empathy was inspired by and adapted from Davis' own organizational model of empathy.
} 
he seems to assume. Empathy and empathy education as presented by Morrell remain unreliable resources in our efforts to counter exclusion in deliberation.

\section{2.ii Projection Bias}

Besides the natural limits and variation of people's predispositions to empathize (both cognitively and affectively), another risk that reduces the practical value of empathy is that of misplaced or incorrect feelings of empathic concern that result from perspective taking. Even when people are likely or eager to engage in cognitive role taking (perhaps because of a predisposition to do so), the desired outcome - what Morrell calls "empathic understanding" - is far more difficult to attain than Morrell believes. In this section, I demonstrate how perspective taking often involves projecting one's own views onto another. Given this propensity for projection, empathy cannot be assumed to provide an accurate picture of the others' point of view to be included in deliberation. Thus I believe we should recognize the possibility of an additional outcome which Morrell does not include in his model: incorrect judgments regarding someone's feelings or motivation.

Evidence in social psychology regarding perspective taking points to the difficulty we have accurately imagining the perspectives of others or even our own future selves. Social psychologists Leaf Von Boven and George Loewenstein (2005) identify what they call the “empathy gap," which divides imagined perspectives from actual perspectives. According to their research, the source of this empathy gap is an egocentric bias in perspective taking that leads individuals to judge others in light of their own thoughts, feelings, or social context. When imagining the perspectives of others, people show significant bias in favor of their own feelings at that particular moment. "Judgments of others are made in comparison to the self, in service of 
the self, and in the direction of the self' (Von Boven and Loewenstein 2005, 293). People base their judgments and predictions about others on themselves "even when they have evidence that their own reactions are anomalous and even when they should recognize that their own experiences are of limited relevance - for example when others' experience is blatantly different from their own" (Von Boven and Loewenstein 2005, 288). Von Boven and Loewenstein explain that this projection is unavoidable - our own judgments are often our best source of information regarding how others would judge or think in a particular context $(2005,297)$. In other words, projection of our own thoughts onto others is the only way for us to engage in imaginative rol taking, and yet it does not adequately inform us about the actual perspectives of others.

Iris Young (2001) identified the dangers of formally incorporating empathic perspectivetaking in deliberation, dangers that stem from the errors and bias of projection. Drawing from Anita Silvers' work (1994), Young vividly demonstrates these dangers with the example the Oregon Health Plan in the 1990s. The Oregon Health Plan was designed in such a way that it disqualified disabled patients from certain treatments because their lives were considered less valuable than those of non-disabled patients. Oregon public policy makers devised their plan with the aid of a telephone survey, asking able-bodied respondents to imagine having a disability. These respondents frequently claimed, "they would rather be dead than confined to a wheelchair" (Silvers 1994, 159). Horrifically, "this claim was the grounds for a political judgment that health services for people with disabilities would not be subsidized in the same way as those for able-bodied people" (Young 2001, 209). Ultimately these regulations were found to violate the Americans with Disabilities Act. 
Just as Morrell would recommend, the participants in the survey "empathized" in the sense of imaginatively taking the perspective of disabled Oregonians. The participants, however, failed to "maintain a healthy distinction between themselves and others," which Morrell's model requires of citizens $(2010,167)$. But, as Von Boven and Loewenstein's findings regarding the empathy gap show, maintaining the distinction between ourselves and others when engaging in perspective taking may, in fact, be impossible. Though they drew incorrect conclusions about others' perspectives, the participants in the Oregon survey may have empathized to the best of their ability. And the process led to outcomes that Morrell does not discuss in his model, namely, incorrect assessment, a failure of empathic understanding, and misguided and unsolicited empathic concern. This example of the empathic process demonstrates the potential for perspective taking to lead to incorrect judgments and misunderstandings of how others feel. Here we see the danger of assuming that the outcome of empathic processes actually reflects the other's view.

In response to the problem of projection, proponents of empathy would remind us that exercises in perspective taking must never replace actual voices in deliberation (Morrell 2010, 166). For example, Krause points out that "We can imagine the sentiments of others much better if they are able to tell us about them, after all" $(2008,113)$. Accordingly, the outcomes of perspective taking should inform deliberation, not replace it. Similarly, deliberation itself should inform perspective taking. Insofar as all citizens have a chance to present their own view and speak for themselves, we minimize the risk of incorrectly projecting our own view onto another. Morrell explicitly cites actual deliberation as the appropriate corrective to the problem of projection $(2010,167)$. In the case of the Oregon Health Plan, this argument would go as 
follows: if lawmakers compared the imagined perspectives of able-bodied respondents to the actual beliefs and opinions of disabled citizens in deliberation, the thought experiment would have been quickly debunked. Empathy's value remains as a supplement to deliberation, not a replacement for it.

I maintain, however, that the risk of inappropriate projection limits the practical value of empathy, even when role taking is included only as a supplement to deliberation. Projection is often inextricably bound up with perspective taking, leading to distortions and misperceptions of others' points of view. And, as Young explains, discussion will not always correct for these errors in empathy. The risk remains that a person, having engaged in perspective-taking, and presuming to have learned the others' perspective, may be less likely to listen in deliberation, especially if she is accused of having gotten their perspective wrong (Young 2001, 215). As Young explains "if you think you can look at things from their point of view, then you may avoid the sometimes arduous and painful process in which they confront you with your prejudices, fantasies, and misunderstandings about them, which you have because of your point of view" $(2001,215)$. The problems with relying on perspective taking persist even when it is incorporated alongside discussion:

If you enter into a dialogue with all the best intentions of taking the other people's perspectives and then in the course of the discussion they express anger and frustration at you for misunderstanding their position, you are likely to become defensive and shut down the dialogue (Young 2001, 215).

Not only are our attempts at perspective taking often biased, resulting in misguided empathic concern or empathic misunderstanding, these attempts have the potential to truncate deliberation 
itself. In presuming to have understood another we are more likely to dismiss her attempts to explain her perspective.

Furthermore, deliberation cannot correct the errors of misplaced or misguided empathy when it comes to groups who are excluded from deliberation in the first place. In these cases, the empathy approach offers no recourse for ensuring that marginalized voices will be heard. This brings me to the third limit of an empathic approach to improving deliberation, namely the difficulty we have empathizing with people most different from ourselves.

\section{2.iii Selective Empathy}

Empathy is meant to improve deliberation by making it more inclusive. But because people tend to empathize selectively - and most often with people who are similar to them empathy cannot reliably improve the initial problem of selective listening and exclusion.

This third challenge to empathy occurs when the subject and the target of empathy have little in common. Morrell acknowledges our limited ability to engage in perspective taking and feel empathic concern for those who are different from us. He claims, however, "that while in general people tend to empathize more with those who are familiar or similar to them, people high in empathic predispositions are more likely to empathize with those who are less familiar and similar" $(2007,398)$. But to address these differences in empathic predispositions, Morrell offers the vague and unsatisfactory recommendation that the answer to the limits of empathy among citizens is creating more empathic citizens.

In her own attempt to respond to concerns regarding our ability to sympathize across difference, Krause points to the importance of empathy occurring alongside deliberation itself. Specifically, she argues that the limits to empathy for those who are different can be mediated 
through deliberation. Sympathy and deliberation are bound together in a mutually beneficial loop whereby deliberative encounters expand the faculty of sympathy (S1). Meanwhile S1 feeds back into deliberation, making citizens open to more people in discourse. Krause explains, "Because sympathy can extend only as far as does our awareness of others' sentiments, the sentiments of marginalized persons - those whose identity or status sets them outside the majority's frame of reference - may tend not to register within the generalized standpoint of average citizens" (2008, 113). The sympathetic imagination helps to bring some concerns and issues to the attention of the polity, but such an imagination does not necessarily extend to the most marginalized. Krause maintains however, that the sympathetic imagination can be expanded to reach excluded minorities precisely through the process of deliberation. "The access to public deliberation that individual rights protect for members of minority groups facilitates such communication and supports regular contestation and debate, which extend the reach of the imagination and influence the contents of our judgments accordingly" (Krause 2008, 113).

This mutual reinforcement of deliberation and sympathy, however, does not adequately correct for the marginalization of groups and individuals who are simultaneously excluded from the majority's sympathies as well as their deliberative practices. People who have immigrated to the United States illegally, for example, are often denied both sympathy and access to deliberative forums. The denial of sympathy for undocumented immigrants often originates in the belief (whether true or not) that they freely chose to come to the U.S. illegally, knowing they would be denied certain rights and protections. Contrary to Krause's claim, this sympathetic exclusion is never corrected as these immigrants are also excluded from deliberative forums. Undocumented immigrants are prohibited from voting, holding office, and other formal political 
forums, while also being discouraged from speaking up in informal deliberative settings due to the threat of detection and deportation.

Thus Krause's virtuous circle of wider empathy and more inclusive deliberation turns into a vicious cycle for groups that are excluded from both empathy and deliberation. This can be the case not only for people who have immigrated to the U.S. illegally, but also closeted gay men and women, victims of rape, victims of gang violence, felons, and numerous other groups who are empathically and politically marginalized. Empathy remains beyond our reach precisely when the problem of exclusion and dialogical closure is most acute.

\subsection{Why Empathy Fails as a Second Best Solution}

Despite the great difficulty we have in imagining others' perspectives, some might argue that empathy is at times a good second-best solution to the persistent problem of exclusion in democratic discourse. In Reflective Democracy, Goodin wrote of the importance of empathy in deliberation especially in representing otherwise "mute interests" (2003). Ideally, all people would speak for themselves, but when social or political inequalities or exclusions prevent equal voice, Goodin thinks that imagining others' perspective is a good alternative or second-best solution.

Goodin explains that "it is undeniably hard to imagine ourselves into the place of a homeless person or a Kurdish peasant, much less into the place of an orangutan or of people a thousand years from now. Still, imperfect though our imagination might be, we will almost certainly be more successful in our imaginings than such agents would be in speaking for themselves in the councils of state" $(2003,14)$. 
Similarly, Frazer explains Herder's position regarding the importance of empathy or Einfühlung when studying ancient peoples. "The study of human beings is, for Herder, not primarily an exercise in reason but an exercise in imaginative Einfühlung, the sort of empathetic insight that would be developed by later German thinkers such as Max Weber as 'understanding' (Verstehen) - the key to all adequate social inquiry" (2010, 155). Herder, as a historian, was imagining the perspective of people who were no longer able to speak for themselves. But in adopting this practice of empathy to contemporary politics both Frazer and Goodin make a false equivalence between subjects incapable of speaking (e.g. animals, the dead, the unborn) and present, but silent, or ignored subjects.

Contrary to Goodin's and Frazer's positions, I argue that given the difficulty people have in imagining how others feel and the risks associated with getting the perspectives of others wrong, we should be wary of taking an empathy-centered approach to improving deliberation even as a second-best solution to the problem of exclusion. Imagining the interests of the politically excluded or voiceless is not a suitable stand-in for actual deliberation even if only temporarily. In fact, doing so may obscure and exclude claims of injustice that do not fit easily within the majority's empathic imagination. But what's worse, our efforts to empathize and imagine the perspective of the marginalized give us a false sense of inclusion that actually obscures the exclusion itself.

Up until now I have challenged empathy as an impractical strategy for making deliberation more inclusive. Empathy may still be defensible, however, as a deliberative ideal. Supporters of empathy could accept the immediate impracticality of empathy as a strategy for promoting greater inclusion, and still argue for empathy as an ideal toward which we should aim. 


\subsection{Empathy as a Flawed Ideal}

According to Krause and Morrell, empathy is thought to assist in ensuring that the procedures and conditions of communication are inclusive of all perspectives. I argue, however, that the architects of the empathic turn propose a mechanism of inclusion that is at least partially at odds with the basic tenets of the deliberative model.

Just briefly, I want to call our attention to the distinctiveness of the deliberative model of democratic justice. ${ }^{13}$ As Habermas explains, what makes the deliberative model so distinct from other models of democracy, is that the normative content of democratic debate and decisions comes from "the rules of discourse and forms of argumentation" and not "the concrete ethical substance of a specific community"(1994, 6). Democratic decisions are judged according to whether they come from fair and equal communication and not whether they accord with the dominant community's conception of the good.

The rules of democratic discourse require that all citizens' perspectives and preferences be included. Communication among citizens creates "influence," and through elections and other media this influence becomes "communicative power." Communicative power is transformed into "administrative power" through legislation (Habermas 1994, 8). It is the realization of this process and the inclusion of relevant perspectives in communication that together ensure laws reflect the opinion and will of all citizens.

${ }^{13}$ Though not Habermas's final word on what he describes as a discursive or proceduralist model of democracy, "Three Normative Models of Democracy" remains one of the clearest, most concise, and systematic accounts of the distinctiveness of deliberative democracy. For that reason, I refer to this paradigmatic essay to show why empathy fails as a specifically deliberative ideal. 
Even when successful, however, empathy does not sustain the fair and inclusive procedures of democratic discourse. Within the context of deliberation, empathy's reliance on commonality has three major problems. First, appeals to empathy demote questions of justice to questions of a particular community's norms and values. Second, empathy distracts citizens from the need to listen to one another. And third, empathy obscures differences that persist even after successful appeals to empathy have identified points of commonality.

\section{4.i The Ethical Constriction of Discourse}

When empathy becomes either the starting point or the goal of deliberation, we run the risk of demoting questions of justice to questions of a particular community's conception of the good. An example of this demotion through empathy can be seen in Obama's endorsement of same-sex marriage in 2012.

President Obama came out in support of legalizing same-sex marriage in the United States in May 2012. In an interview, he cited empathy (both imagined perspective-taking and empathic concern for gay couples) as the driving force behind the evolution of his position (Lithwick 2012). Many people celebrated Obama's position as well as the empathic justification he provided for his shift of opinion. I believe that closer inspection shows that Obama's appeal is more in line with a republican-communitarian model of democracy which relies on a thicker conception of the good than is assumed in the deliberative model. Obama's appeal to empathy, ultimately, demotes same-sex marriage from a question of justice to a question of communal conceptions of the good.

After cloaking the justification of his position in the language of empathy, Obama explained: 
At a certain point, I've just concluded that-- for me personally, it is important for me to go ahead and affirm that-- I think same-sex couples should be able to get married. And what you're seeing is, I think, states working through this issue-- in fits and starts, all across the country. Different communities are arriving at different conclusions, at different times. And I think that's a healthy process and a healthy debate (Obama 2012).

It was precisely the inherent gradualism and non-universalism of Obama's empathic support for gay marriage that conservative columnist Charles Krauthammer celebrated in his Washington Post op-ed on the subject. Krauthammer much preferred the empathy-based argument compared to a "rights-based" appeal to justice that he condemned as too radical (2012). Krauthammer explained that Obama's "empathy argument both encourages mutual respect in the debate and lends itself to a political program of gradualism. State by state, let community norms and moral sensibilities prevail" (2012). In other words, legalize same-sex marriage in communities where the norms and moral sensibilities allow the majority to empathize with the minority of gay and lesbian couples. But in those communities where the norms do not allow same-sex couples to be seen as empathetic characters, banning same-sex marriage would be legitimate.

As this example illustrates, empathic approaches to democracy have the potential to lead to an ethical constriction of political discourse. Though ethical discourse plays an important role in politics, ethical questions must remain subordinate to universalistic moral questions. According to Habermas's terminology, moral questions deal with universal justice and not the individual preferences or established values of a particular community, which are the concerns of ethical questions $(1994,5)$. When discourse is ethically constricted, instead of asking what is the "just course of action," citizens ask what is the "best course of action" for this particular community given our own particular commitments. The procedures of fair and inclusive 
communication must transcend particular conceptions of the good in order for deliberative models to generate legitimate decisions in diverse societies where people have divergent and potentially conflicting communal norms and values. Appeals to empathy of the sort Obama provided in May 2012 rely on a thicker ethical consensus than the deliberative model of democracy provides. By contradicting the priority of the right over the good, empathy proves to be an inappropriate ideal for deliberative democracy.

When political questions are discussed, citizens must listen with or without empathy. Successful perspective taking and feelings of empathic concern for a mother on welfare, a wounded veteran, a same-sex couple, or a member of any other politically marginalized group are commendable, and may even facilitate deliberation. But when pursued as a means of democratizing discourse on relevant policy issues, appeals to empathy run the risk of demoting moral questions to ethical ones.

\section{4.ii The Undermining of Listening}

The second challenge to pursuing empathy as an ideal of deliberation is that empathy distracts citizens from the need to actually listen to one another. Focusing on our ability to imagine how another feels renders the need to listen less urgent. Therefore, even when done correctly, empathy fails to support and foster the communicative procedures, especially listening, that models of deliberation require.

Even when we are able to feel for or even with someone, we can never actually feel as they do. To navigate rather than collapse the distance that exists between citizens, we must listen to one another with an initial humility. But this distance is ignored by proponents of empathy, including Krause who claims that in deliberation, "it is not enough for me to know cognitively 
what [your sentiments] are; they must also be (or become) objects of concern for me, or at least they must connect up with concerns that I have" $(2008,164)$. When we focus on generating empathy among citizens, requiring your concerns to actually become my concerns, we ignore the distance that remains between ourselves and even the most empathy-worthy characters. The need to actually listen to others is muted when we assume that their sentiments echo our own.

In her book on democratic listening, Susan Bickford explains that "I try to experience the world as you construct it for me, but this is not the same as experiencing it as you do; it is still, always, for me" (1996, 147). Good listening practices, especially listening-with-humility, are vital to democracy, especially in deliberative models where actual discussion and argumentation are required for decisions to be made. In such a context, it is crucial to remember that my experience must always be mediated by my attempt to communicate with you.

Young speaks precisely to the importance of acknowledging this mediation in deliberation when she challenges the idea that "moral respect entails being able to adopt the standpoint of others" $(2001,208)$. In line with her commitment to inclusive communication as the legitimating force of democratic decisions, she also emphasizes the importance of listening over empathy. Distinguishing "between taking the perspectives of other people into account, on the one hand, and imaginatively taking their positions, on the other," Young endorses the former and rejects the latter $(2001,206)$.

Practically speaking, there is a redundancy to empathy in that it is available to us precisely when it is not needed - when we share common interests and have common ethical norms and are therefore already likely to listen to a particular point of view. Conversely, empathy is out of our reach precisely when the threat of dialogical closure is most acute, when 
citizens encounter those most different from themselves and conversation is most likely to breakdown.

Even in those instances when empathy is successful, the need to listen is muted insofar as we assume that we have access to others' thoughts or feelings, or assume that their sentiments echo our own. Good listening practices are absolutely vital to democracy, especially deliberative democracy where actual discussion and argumentation are the bases of legitimate collective decisions. Though empathy may promote deliberate-ness, it may actually undermine the deliberative-ness of democratic decision-making. Rooted in imagined perspective taking rather than authentic engagement, empathy represents a premature consensus that undermines the very possibility of reaching legitimate decisions through political discourse. Empathic approaches fail to foster the kind of listening needed in the presence of deep differences among citizens.

\section{4.iii The Obscuring of Difference}

Focusing primarily on our commonalities and assuming that we can fully understand or even imagine how another feels, a reliance on empathy will systematically exclude and devalue concerns and perspectives that do not map on to the interests and ethical priorities of the majority. Furthermore, even successful attempts at empathy run the risk of obscuring the exclusion that persists even after empathy for some has been achieved. Not only does the majority remain deaf to these others' concerns, but attempts at empathy also give the majority a false sense of inclusion, blinding them to persistent exclusion.

The work of Bickford and Young gives us reason to be skeptical of empathy for its inability to accommodate difference in democracy. But their critiques regarding the failure to accommodate differences extend to the deliberative model as a whole (Dryzek 2002). These 
critics challenge deliberative models for privileging consensus at the expense of respecting difference. For them, empathy would represent a kind of proto-consensus that facilitates the ultimate consensus at which deliberative models are aimed. Difference democrats must reject empathy precisely because it is compatible with deliberation's objectionable drive for consensus and disregard for difference. In order for a decision to be reached, differences must be collapsed through appeals to empathy.

Contra these critics, however, I would argue that the empathic approach has a unique disregard for difference that actually contradicts deliberative democrats' commitment to fair (and actual) argumentation. For example, in creating a democratic model that does not assume shared interests or shared conceptions of the good, Habermas makes room for deliberation across difference. Appeals to empathy, however, which ignore these differences from the start, undermine the potential for agreement or even discussion across differences. A truly deliberative ideal must show citizens the importance of reorienting themselves toward their fellow citizens in a way that allows deliberation to occur among people with different interests and different ethical conceptions of the good.

Appeals to commonalities - whether real, imagined, or artificially imposed - paper over the division and disagreement that exist between citizen-listeners. Though this is a charge that some have leveled against deliberation as a whole, I maintain that the deliberative model is not inherently inhospitable to the recognition of disagreement and respect for differences among citizens. In fact, the need for discourse only arises after an initial disagreement interrupts ongoing communicative action. And as Stephen White and Evan Farr explain, the charges that Habermas's model amounts to a "great consensus machine" might be unfounded $(2011,33)$. 
White and Farr argue that the initial moment of disagreement (or no-saying) that interrupts communicative action cannot be reduced to merely a "discursively framed normative argument" intended to bring about renewed consensus $(2011,40)$. Importantly, they identify the moment of no-saying in Habermas as also representing a diffuse "existential taking-of-a-stand against the normative force of a dominant form of life" $(2011,38)$. In their view, Habermas's brand of deliberative democracy is more hospitable to deep difference and disagreement than many critics claim. Through their reinterpretation of no-saying in Habermas, White and Farr show that Habermas's model of deliberation acknowledges the deep, inarticulate, and existential differences that exist between people, produce disagreement, and inform our communicative interactions.

Like White and Farr, I maintain that deliberative democracy can enhance legitimate political decision-making while also recognizing, accommodating, and protecting differences among citizens. But an orientation of greater openness towards difference is not automatic on the part of citizens, and it cannot be achieved through appeals to empathy. Once achieved, however, such an orientation can foster successful deliberation that addresses many of the concerns that led difference democrats, including Young and Bickford, to criticize the deliberative model for its inattention to difference.

\subsection{Conclusion}

In this chapter I have focused on problematizing the link between empathy and inclusion. The empathy approach suffers from significant practical challenges, including our inability to achieve empathic understanding or feel empathic concern for those who are most different from us. These practical limits make empathy an unlikely resource in democratic practice. 
Furthermore, empathy fails as an ideal or goal towards which to aim in deliberation. By focusing on commonalities, the empathy approach distracts us from the challenges of ensuring fair discussion and reaching agreement in the absence of common perspectives, experiences, or interests. Contrary to its aims, the empathy approach exacerbates the concerns of difference democrats rather than alleviates them.

The remainder of the dissertation offers an alternative to the empathy approach to improve deliberative practices. In the place of empathy, I propose a "difference approach." In the next chapter I explore how the recognition of difference can sustain deliberative practices among citizens. 


\title{
Chapter 3. The Difference Approach to Improving Democratic Deliberation
}

\author{
A wonderful fact to reflect upon, that every human creature \\ is constituted to be that profound secret and mystery to every \\ other. A solemn consideration, when I enter a great city by night, \\ that every one of those darkly clustered houses encloses its own \\ secret; that every room in every one of them encloses its own \\ secret; that every beating heart in the hundreds of thousands of \\ breasts there, is, in some of its imaginings, a secret to the heart \\ nearest it!
}

\section{-- Charles Dickens, A Tale of Two Cities}

The challenges to democratic discourse in pluralistic societies are significant. Ensuring that citizens listen to others and actually take alternative viewpoints into consideration is necessary for deliberative models to deliver on the democratic promise of equal consideration. Recent accounts of how to ensure fair communicative practices have focused on the importance of fostering empathy among citizens. Krause, Morrell, and Frazer, for example, argue that empathy in the form of perspective taking as well as feelings of empathic concern among citizens can make deliberation more inclusive. For them, the path to meaningful engagement between citizens goes through empathy. Expanding the bounds of our empathic imaginations and sentiments is supposed to democratize deliberation by making it more inclusive.

In this chapter I theorize about how to cultivate openness and therefore achieve inclusion, independent of feelings of empathy. I look for alternatives to empathy, in part, because empathy is not easy to achieve and is rarely felt in uniform or universal ways. We feel empathy most naturally and effortlessly for those who are like us, those who share our opinions and perspectives. But the problem of dialogical closure is most acute precisely when dealing with those who are unlike us, when empathizing is also most difficult. Though empathy - when 
successful - may be sufficient for bringing about greater openness among citizens, I argue that it is not necessary. I am interested in finding resources to induce openness precisely when empathy is out of reach.

To introduce the benefits of an alternative to the empathy approach, I appeal to continental philosopher, Martin Heidegger's later writings on the limits of mutual understanding that are inherent to language. With the help of Heidegger, I consider how experiences of difference help us to acknowledge the limits of "knowing others" and "being known to others," and can therefore humble people in their encounters with fellow citizens. I show that a more democratic source of dialogical openness may be our experiencing the limits of communication and realizing our inability to fully express ourselves or understand each other in the presence of the deep differences that separate us. I call this the "difference approach" to dialogical openness. The realization of the limits to mutual understanding in the presence of deep difference opens us more vividly to the ways in which we are inconspicuously limited in our opinions, our basic understanding of issues, and our relationship to the democratic community. This sort of opening cannot occur if we imagine only our commonalities and assume that we can know or feel how another feels. To foster greater openness, we should relish our differences instead of moving quickly beyond them for the sake of imagining commonalities. In Chapter 4, I consider how aesthetic expression can foster dialogical openness by calling our attention to differences rather than commonalities.

This chapter aims to accomplish two things. The primary aim, as I have just explained, is to develop an approach to fostering dialogical openness that does not rely on empathy. In developing this alternative, however, I also articulate a novel way of understanding difference as 
a resource in democratic deliberation. The other aim of this chapter, then, is to situate the difference approach within the broader literature regarding the role of difference and democracy. The difference approach that I propose contributes to an established tradition in political theory that emphasizes both the fact and value of difference in democracy (for summaries of this tradition, see Dryzek 2002 and Dahlberg 2005).

Like the "difference democrats" with whom I engage in this chapter, I acknowledge the inevitability of deep differences and conflicts among citizens. The difference approach takes seriously the fact of difference in large, pluralistic democratic polities. This approach aims to make models of democratic deliberation more accommodating to difference and disagreement. The difference approach, however, goes beyond simply recognizing the fact of difference, pointing to the ways we might think of difference as a resource and facilitator of democratic deliberation. In my view, difference is not merely an obstacle to deliberation, nor is it simply a background condition of contemporary politics that models of deliberation must address. The power that I believe differences can hold for democratic deliberation comes from its potential to open citizens up or at least highlight the ways in which they are closed off to others.

\subsection{Democracy and Difference}

The importance of difference in democracy has been noted since critics took issue with deliberative democrats' perceived overemphasis on consensus, unity, and agreement. As I explain in Chapter 1, "difference democrats" are "those who stress the need for democratic politics to concern itself first and foremost with the recognition of the legitimacy and validity of the particular perspectives of historically-oppressed segments of the population" (Dryzek 2002, 57). Opposed to these difference democrats are critics who claim that a politics of difference 
produces divisions and conflicts of self-interest. Difference democrats resist this line of thinking, pointing out that social group differentiation exists whether or not we pay attention to it.

Whereas attention to difference does not cause division, an inattention to such difference renders us unable to account for or even notice "continuing patterns of privilege, disadvantage, and exclusion that structure opportunity and capacity in modern societies" (Young 1997A, 388-389).

Although difference democrats do not make up "a self-consciously unified school of thought," all difference democrats agree that differences in perspectives, values, and interests are an unavoidable fact of politics and therefore can only be ignored with damaging and exclusionary effects (Dryzek 2002, 57). In this view, democracy always takes place within a context of difference and disagreement. This fact of difference presents a serious challenge to reaching consensus or agreement; the democratic potential of deliberation is bounded by difference.

Though united in the recognition of the fact of difference, difference democrats do not all agree on the best way to achieve the promise of democracy in light of this fact. Specifically, they do not agree on the role that deliberation can or should play in democratic decision-making. For Rawls, the fact of difference is an obstacle to deliberation that must be overcome in order to reach legitimate decisions through public reason. For others, especially agonistic democrats, the fact of difference gives us reason to be deeply suspicious of deliberative models of democracy. But still other difference democrats maintain that the democratic potential of deliberation can actually be fostered by difference. For them, the fact of difference is neither something to be transcended nor is it something to be merely accepted. While difference certainly poses a challenge to the deliberative ideal, these theorists argue that difference can also be seen as a 
resource to help meet the very challenge it presents. Young, for example, remains committed to a modified ideal of democratic deliberation, helping us see the ways that difference, especially differences in perspectives, can actually facilitate legitimate decisions. Following, Young, the difference approach that I propose here takes into account both the fact of difference in democracy and the value of difference in deliberation.

Identifying the value of difference in this case does not mean that, all else equal, we should try to encourage greater diversity than might already exist. In other words, recognizing the value of difference does not require us to introduce difference where it is otherwise absent. The question I am asking is not whether more or less diversity is desirable as such. Instead the question is, given that we are operating always already in the presence of difference (this is what the fact of difference entails), what is the best way to accommodate different perspectives, ideas, and values?

In order to situate the difference approach among other accounts of the fact and value of difference in democracy, I compare three classic accounts of the function of difference in democratic theory, especially as it relates to democratic deliberation. Specifically, I compare the work of Rawls, Mouffe and Young. Expanding on these, I show how my approach points to a previously unarticulated way that differences can serve as a resource in democratic deliberation by promoting listening and 'uptake' in democratic encounters.

\section{1.i Fact of Difference}

I offer John Rawls's treatment of difference in Political Liberalism as the paradigmatic case of taking difference to be a mere fact of modernity. As I explain in Chapter 1, Rawls discusses the fact of pluralism as a defining condition of modern liberal democracies. Rawls goes 
on to design his preferred democratic decision-making and deliberative procedures with the fact of difference in mind, specifically so as to minimize the disruptive effects that differences will have on democratic decision-making. For Rawls, the fact of difference is something to be controlled, stabilized, and ultimately overcome.

Radical democrat Chantal Mouffe criticizes Rawls's view of difference. Mouffe explains that the main forms of liberal pluralism generally start by "stressing what they call the "the fact of pluralism' and then proceed to find procedures to deal with differences whose objective is actually to make those differences irrelevant and to relegate pluralism to the sphere of the private" $(1996,246)$. In contrast to Rawls, she refuses "the objective of unanimity and homogeneity which is always revealed as fictitious and based on acts of exclusion" $(1996,246)$.

Like Rawls, Mouffe seeks to incorporate the fact of difference into her understanding of democracy. Yet she disagrees with Rawls's understanding of difference as an obstacle to overcome insofar as she gives a "positive status to differences" (1996, 246). Mouffe understands difference as permeating all aspects of political life. There is no way around it. "Instead of trying to erase the traces of power and exclusion," which would be impossible given Mouffe's understanding of the fact of difference, "democratic politics requires bringing them to the fore, making them visible so that they can enter the terrain of contestation" $(1996,255)$. Difference and disagreement are inevitable. An alternative to the politics of difference could only be brought about through exclusionary and homogenizing practices.

In light of the fact of difference, Mouffe "rejects the very possibility of a nonexclusive public sphere of rational argumentation where a non-coercive consensus could be attained...such a rejection constitutes an important guarantee that the dynamics of the democratic process will be 
kept alive" $(1996,255)$. Mouffe focuses primarily on the inescapability of difference and the dangers of trying to transcend it. The fact of difference cannot be circumscribed or tamed, and it especially cannot be relegated to a pre-political sphere. Democracy becomes a question of how we might engage with one another in the presence of irreconcilable differences.

Mouffe wants us to relish our differences; overcoming them as Rawls suggests would be impossible. She is not, however, as difference-positive as those who claim that differences can actually serve as a resource in democratic deliberation. In Mouffe's view, agreement is always deferred in the presence of difference and resulting disagreements. Through this reading of the fact of difference, difference presents a devastating challenge to deliberative decision-making.

In short, both Rawls and Mouffe see difference and deliberation as being zero-sum and inherently in conflict with each other. Like Mouffe, Rawls believes that the fact of difference undermines the deliberative ideal. Whereas Rawls tries to get past the obstacle of difference for the sake of deliberation, Mouffe's dispenses with deliberation for the sake of recognizing the fact of difference. Despite their differences, Rawls and Mouffe both see the fact of difference merely as a challenge to democratic deliberation. Whereas Mouffe values difference over deliberation, Rawls values deliberation over the inclusion or celebration of difference. Neither ascribes any positive value to difference in the context of deliberation.

\section{1.ii Difference as a Resource}

Young recognizes the fact of difference as an important challenge to the deliberative ideal. Like Rawls, Young favors a model of democratic decision-making that is centered on political communication among citizens. But like Mouffe, Young opposes attempts to overcome difference for the sake of achieving the deliberative ideal. As Susan Bickford explains, "those 
who take conflict seriously tend not to stress interaction, while those who value interaction tend to underestimate the presence and persistence of conflict" (Bickford 1996, 5). This observation bears out in my analysis of Rawls and Mouffe above. Young, however, sees a potential way out of the fundamental tension between difference and conflict on the one hand and interaction and democratic deliberation on the other. Rather than choosing between democratic deliberation and a politics of difference, Young identifies a way that difference might actually sustain deliberation in pluralistic democratic polities.

Therefore, the fact of difference is neither simply an obstacle to be overcome, nor a challenge that necessarily dooms deliberation to fail. Difference presents a challenge to deliberation, but as Young shows us it also provides us with resources to overcome the very challenge it presents. In an important move, Young defines difference in terms of perspectives. Defining differences in this way allows Young to make the claim that these differences in perspective actually facilitate deliberation rather than merely set the basic parameters or requirements of deliberation (per Rawls) or ultimately undermine our deliberative ends in the first place (per Mouffe).

According to Young, the "inclusion of differentiated groups is important not only as a means of demonstrating equal respect and to ensure that all legitimate interests in the polity receive expression, though these are fundamental reasons for democratic inclusion" $(2001,115)$. The inclusion of difference is not only a means to avoiding repression; it also serves two primary functions that facilitate deliberation: "First, it motivates participants in political debate to transform their claims from mere expressions of self-regarding interest to appeals to justice. Second, it maximizes the social knowledge available to a democratic public, such that citizens 
are more likely to make just and wise decisions" $(2001,115)$. By piecing together the diverse social perspectives available in our polity due to the fact of difference, we are able to get a more complete view of our shared world. Audre Lorde speaks to this view of difference serving a certain informational or epistemic function when she writes that encountering those who are different from ourselves "enables us to descend into the chaos of knowledge and return with true visions of our future, along with the concomitant power to effect those changes which can bring that future into being." (1984, 111-112).

In such an account of the value difference in democratic communication, Young focuses primarily on the effect that difference has on democratic inputs. "Pooling the situated knowledge of all social positions can produce” an “objective understanding of the society...” (Young 2001, 117). For Young, this objective understanding does not come from bracketing and excluding "differences, but by communicating the experiences and perspectives conditioned by them to one another" $(2001,83)$. The communication of differences fosters "objectivity" in this sense by correcting biases that occur when we only consider one point of view. The sharing of different perspectives increases "the store of social knowledge available to participants" (Young 2001, 83). In her interpretation of difference as a resource, however, Young seems to miss the fact that what makes particular perspectives so valuable for democratic discourse is precisely what makes them difficult for others to understand. We cannot hold two different perspectives at once. And expanding the pool of knowledge available to citizens is only valuable if we can ensure they will actually refer to it. How can this be done?

In her discussion of difference as a resource in democratic communication, Young begins to hint at the power of difference to democratize uptake as well as input, in other words, to make 
citizens (and not just the procedures of deliberation) more receptive. Beyond the informational value of increasing the pool of social knowledge available to citizens in deliberation and encouraging citizens to make claims in terms of justice, Young gestures at a third feature of dialogue across difference that makes it a valuable resource for making just decisions:

Confrontation with different perspectives, interests, and cultural meanings teaches individuals the partiality of their own, and reveals to them their own experience as perspectival. Listening to those differently situated than myself and my close associates teaches me how my situation looks to them, what relation they think I stand to them (1997A, 403).

Again, we see the claim that difference serves as a resource by pluralizing the input or information available in deliberation. But with this third feature, Young suggests, in passing, that difference may have an effect on listeners as well, making them more receptive to others. In the remainder of this chapter, I develop this line of thinking. I argue that difference in democracy has value beyond democratizing input. But this potential of difference is only visible if we understand citizens in their role as listeners as well as speakers. Only after accounting for the importance of listening in democratic theory, especially deliberative democracy, can we fully understand the value of difference as a resource for making democratic deliberation more inclusive. Unfortunately, however, listening is often ignored in favor of privileging speaking. Perhaps because of the difficulty we have guaranteeing or even measuring listening or receptivity among individuals in discourse, this important theme has been all but ignored in theories of democratic deliberation.

\subsection{Listening}

Attention to procedures of listening is scarce in theories of democratic deliberation. Those democratic theorists who do attend to the importance of listening do so within the context 
of agonistic democracy. Bickford, for example, puts the value of listening at the center of her agonistic "understanding of politics that stresses its conflictual and contentious character" (Bickford 1996, 2). Dispensing with the goal of consensus or mutual understanding, Bickford bases her theory of democratic communicative interaction on the "presence of listening" (1996, 18). Given what Bickford takes to be the fact of perpetual conflict and the impossibility of consensus, she offers listening as a more attainable goal than consensus or understanding. While Bickford incorporates listening into agonistic democratic theory, I seek to provide an account of the importance of listening to be used within the framework of deliberative democracy. Rather than using listening as a substitute for agreement or understanding, I want to explore the ways that listening can facilitate mutual understanding. How might the presence of attentive listening actually enhance the possibility of greater agreement through deliberative encounters? How might we meet the condition of listening that most theories of deliberation assume to be automatic.

In our attempt to improve procedures of deliberation, an overly narrow focus has been on procedures of deliberation-as-speech. One of the implications of this is that we miss the duality of the ideal of inclusion; being heard is as important as being able to speak. Furthermore, we miss the potential for differences to guarantee the former aspect of inclusion and promote more attentive listening among citizens. As Nancy Love points out in her book Musical Democracy, deliberative democracy "privileges speaking over listening and performers over audiences" $(2006,82)$. This preference for speaking over listening is highly problematic insofar as equal and democratic communication can only occur between "a speaker and a listener" and not simply “two speakers" (Schweickart 1996, 317, as quoted by Love). Accounting for the listening 
component of deliberative procedures is crucial if we are to achieve the legitimate outcomes that deliberative democrats seek.

The difficulties of observing improvements to listening and openness have led to an unfortunate inattention to such an important concept. As anyone who has led a seminar knows, engagement-as-speech is infinitely easier to observe and quantify than engagement-as-listening. I would argue that because of the relative ease with which we can identify procedures that are inclusive of speech rather than listening, the former is often the preferred measure of or proxy for healthy deliberation. The inclusion of all relevant and willing speakers is certainly a necessary condition for deliberation; but it is not a sufficient one. Speakers must also have an attentive audience.

Even "silence" which is readily observable, and perhaps the most obvious condition for and correlate of democratic listening and openness, is just as likely to signify withdrawal from discourse as it is meaningful engagement (Bickford 1996, 153). And the transformation of opinion is a similarly unsuitable measure of engagement-as-listening. For example, I may attentively listen to a neo-Nazi without being swayed by his position and, in fact, careful listening my increase or harden my opposition to his views.

One possible approach to judging the level of engagement-as-listening would be to measure participants' ability to recall someone's opinion. While this may be a good start in terms of measuring an individual's listening skills (and short-term memory), it will not give us a sense of the extent to which participants gave serious consideration to others' opinions. The challenges political scientists and theorists face in identifying capacious listening practices is problematic 
insofar as it has led to the anemic investigation into the important question of improving listening in deliberation.

In light of these challenges, we might be advised to turn to Diana Mutz's Hearing the Other Side. Despite its title, Mutz's Hearing the Other Side employs only a basic threshold of "hearing," which actually boils down to mere exposure to alternative viewpoints. Mutz avoids the question of receptivity or listening and her approach betrays a continued focus on deliberation-as-speech. For Mutz, "hearing the other side" amounts to the mere presence of dissenting opinions. In the end, rather than studying hearing, listening, or engagement, Mutz studies the impact of exposure to different opinions. Could such exposure prove a fruitful standin or proxy measure for listening? According to Mutz, "cross-cutting exposure" occurs in "crosscutting networks" wherein "members of one's social network hold views different from one's own” (Mutz 2006, 101). A network will bring about cross-cutting exposure to the extent that "political discussions with non-like-minded others are taking place within these networks" (Mutz 2006, 102). Again, Mutz's operationalization of "hearing the other side" is simply the presence of opposing voices without any mention of hearing.

Importantly, Mutz finds that exposure to opposing views can actually bring about a withdrawal from politics. She identifies a trade-off between diversity and engagement, explaining that the most engaged are those surrounded by like-minded people. And those surrounded by people with opposing political beliefs are actually more likely to be politically withdrawn. Insofar as Mutz finds that the presence of opposing views in citizens' networks has effects on their level of engagement, we might conclude that some sort of uptake or reception 
must be occurring alongside the exposure. But again, Mutz does not discuss the quality of the uptake or describe how it could be measured, let alone improved.

Although Mutz's account of the effects of exposure to cross-cutting views is an important contribution to the study of democratic deliberation, she is unable to give an account of the quality of exposure. And as Michael Neblo et al. find, "even though some aversion to conflict may be widespread, it is hardly decisive with respect to participating in deliberation" (2010, 581). Perhaps the demobilizing effect of exposure to disagreement could be alleviated or better understood if we had an account of what sustained and engaged listening in the presence of disagreement should look like. But, again, because of the difficulty we have guaranteeing or even measuring the listening or receptivity among individuals in discourse, this important theme has been all but ignored among political theorists and political scientists studying democratic deliberation.

In order to conceive of, as well as work towards, the two-sided ideal of inclusion, I argue in favor of reconceiving citizens as listeners. Even if not their primary mode of participation, listening is at least as important as speaking. And as I will show, refocusing on the importance of listening opens new avenues for understanding difference as a resource in democratic deliberation.

Judging deliberation according to whether citizens listened to one another is a lower standard than judging it according to whether consensus is achieved. Similarly, listening is a weaker condition for deliberation than shared interests or a shared conception of the common good. The ability to listen to one another does not presume commonalities or preliminary agreement. Using listening as the standard of deliberation allows for deliberation in the absence 
of common interests or a common good. But although listening is a weaker condition to meet in a pluralistic society, attentive listening is not automatic and we struggle to understand how to bring it about in practice. In the next section, I hope to shed some light on one possible resource available to citizens in deliberation that can help promote dialogical openness and therefore more attentive listening practices.

Building on the work of difference democrats discussed above, I will argue that greater attention to differences among citizens - in opinion, identity, interests, and most importantly perspectives - has the ability to bring about improved listening. The "difference approach" to democratic deliberation that I propose highlights the ways in which attention to differences can work on citizens in such a way as to open them up to deliberation and make them more capacious listeners.

\subsection{The Difference Approach}

Taking what we have learned about the relationship between deliberation, listening, and difference, I want to propose an alternative to the "empathy approach" to making deliberation more open and inclusive. Specifically, I will outline what I call the "difference approach" to improving deliberative practices. I explore how such a disposition can be brought about by the realization of the limits of communication, and our inability to fully express ourselves or understand each other in the presence of deep differences that separate us. The difference approach, though indebted to other discussions of the fact and value of difference, goes beyond existing accounts of difference in democracy to show how difference might democratize uptake as well as input. 
In her understanding of difference as a resource, Young argues for the inclusion of differences, especially differences in perspective as a way to increase the pool of social knowledge available to citizens making decisions. What she misses however, is the fact that what makes your perspective unique and therefore valuable, is precisely what makes it difficult for others to understand and thus likely to be ignored. This tension between inclusion as input and inclusion as uptake undermines not only the value of difference as a resource in deliberation, but the deliberative enterprise as a whole. The more marginalized a person's perspective, the more important it is to be included and yet the more difficulty members of the majority will have in understanding it. To advocate inclusion as input without considering inclusion as uptake misses an important part of the story. The inclusion of minority voices will not ensure that they are meaningfully considered.

Having situated my understanding of difference and democracy within the broader literature on the subject, I turn now to the primary aim of this chapter: demonstrating how the difference approach functions as an alternative to the empathy approach. Rather than coming from empathy and perceived commonalities, I contend that citizens are attuned to the problem of dialogical closure when they are made to feel the intractable divide that can exist between themselves and others. In other words, realizing the need for dialogical openness comes from the acknowledgement and acceptance that we can never completely understand another's position or point of view. Such feelings of difference can help citizens cultivate dialogical openness and its characteristic humility, receptivity, and generosity to others. In contrast, feelings of empathy focus on how I can come to understand or at least imagine how another feels. But only in recognizing the potentially unbridgeable differences between ourselves and others does the need 
to actually listen and engage in discourse become so urgent. While some of the differences might be bridgeable over time, what is key is an appreciation of difference or distance that alerts citizens to the emotional and cognitive work required for engagement to occur.

When made to feel and recognize the deep differences and divides that exist culturally, experientially, and communicatively, citizens are transformed. As a result, they are encouraged to 1 ) accept the provisionality of their own beliefs, 2) recognize their fellow citizens as equal interlocutors, and as a result of these first two transformations 3) delay in her response toward others, creating the necessary space for more adequate discourse to occur. When these three transformations have occurred, citizens are able to cultivate a more dialogically open disposition in order to engage with their fellow citizens in deliberation.

To explain why feelings of difference sustain democratic deliberation by attuning citizens to the need for dialogical openness, I turn to Heidegger. By referring to aspects of Heidegger's understanding of language, we come to see the fact and value of openness in the face of limits to mutual-understanding.

\section{3.i Martin Heidegger, an Unlikely Resource for Democratic Theory}

To defend a politics of difference as a possible source for the openness required in democratic deliberation, I use Heidegger's concept of the world-disclosive power of language. In the same way that William Connolly has appropriated elements of the highly anti-democratic Nietzsche into his theory of agonistic democracy, I aim to reclaim aspects of Heidegger's later work to incorporate into an otherwise Habermasian model of deliberative democracy. Of course, 
this appropriation must remain "critical and distrustful," so as not to overlook the striking incompatibilities of Heidegger's ideas with democracy (White 1991, 31). ${ }^{14}$

Heidegger and Habermas differ sharply in their views of the essential character of language. For Heidegger, language is oriented towards world-disclosure. For Habermas language orients us towards mutual understanding, which allows us to coordinate action through speech. The difference in these two thinkers' views of language originates in Heidegger's rejection of the modern view of the world as something to be controlled (enframed in his words). This modern orientation to the world extends especially to the modern understanding of language as a tool that humans use. According to Heidegger, individuals are embedded in language. He writes, "We do not have language, rather language has us" (Heidegger 1980, 23).

There are important implications of Heidegger's critique of what he calls "the current view" of language, whereby it is "held to be a kind of communication" $(1971,71)$. Unlike Habermas who focuses on the ability of language to coordinate action, Heidegger emphasizes the aesthetic qualities of language and its ability to "disclose being."

As I have shown in Chapter 1, Habermas's theory of deliberative democracy demands that one be open and receptive to the other's words and arguments, but does not provide any explanation of how a greater sensitivity to the other is achieved. Although Habermas's assumptions regarding mutual understanding and consensus are much weaker than critics often charge, he still has a yet unjustified assumption regarding our general "orientation" toward

\footnotetext{
${ }^{14}$ Similarly, I think that it is important to remain wary of the troubling actions Heidegger took (or failed to take) in his own life. In light of these concerns, I share White's conviction that we can employ Heidegger's contributions to political thought "only if we do not forget either the heights or the depths of his thought," including his consenting (even if only for a matter of months) to Nazism.
} 
mutual understanding. Heidegger's understanding of language as poetry can help correct for this unfounded assumption.

For Heidegger, the power of language, understood according to a more aestheticized sense and its potential for world-disclosure, goes beyond its everyday usage. "Language alone brings what is, as something that is, into the Open for the first time" $(1971,71)$. Given existence's embeddedness in language, we cannot understand that which is except through language. Rather than merely coordinating action, language discloses being.

To Heidegger, language not only expresses "what is overtly or covertly intended to be communicated," but has potential that goes far beyond intentional communication $(1971,71)$. Heidegger discredits any sort of instrumentalist view of language insofar as human beings are wholly embedded in language. There is no identity or thought that can exist prior to language. For Heidegger, we operate “'always already' within a pre-reflexive, historically structured, and grammatically regulated understanding of the world" (Kompridis 2006, 33). Language, therefore, is not something simply used by rational beings to communicate previously unmediated thoughts to one another.

I contend that Heidegger's emphasis on world-disclosure shows us the fact and value of openness in language, especially openness in our encounters with that which we cannot fully understand or relate to. According to Heidegger, language shares with art its ability to bring about an "unconcealedness of being," in which we come to know, to see, or to understand a being through language. Importantly, however, this disclosure occurs through the conflict of unconcealment (whereby you come to know something of me) and concealment (whereby I ultimately remain unknowable to you). "Each being we encounter and which encounters us [in 
language] keeps to this curious opposition of presence in that it always withholds itself at the same time in a concealedness" $(1971,52)$. In the same moment that a being is present in the clearing - as in, a clearing in the woods - it remains withheld. Even when we come to see, to understand, or to know something, someone in discourse, that thing or person remains ultimately hidden, incomprehensible, or unknowable as it is distinct from ourselves. As we come closer to comprehending that which is different from us, it is crucial that we acknowledge that which remains forever unknowable. What we see in the clearing only appears against the dark. While we can move elsewhere and perhaps see and understand something new, what we saw before slips back into an occluded state. Although our encounters with others allow us to progressively know more, we can never get to a position that would allow us to see or understand everything or even most things simultaneously.

Heidegger explains that the clearing - the space wherein unconcealedness occurs - "is never a rigid stage with a permanently raised curtain on which the play of beings runs its course" $(1971,52)$. Rather this "open space in the midst of beings" is a "happening," a continual process "that includes the conflict between concealment and unconcealment" (1971, 52). For Heidegger, it is in the recognition of this perpetual play of unconcealedness and concealedness that one comes closer to truth, and in this case, closer to understanding another's being. I argue that recognizing, or perhaps feeling, this perpetual concealment attunes citizens to the problem of dialogical closure. By recognizing that concealment remains even in the appearance of "the clearing" where we encounter others, we can be made more humble and generous, and less acquisitive in our linguistic interactions. 
Even when we are engaging in discourse for the sake of coming to a decision, citizens must recognize that they will never fully understand their opponent's perspective and, likewise, their opponent will never understand their own. Only once these differences and divides are acknowledged and the concealment of another's world is recognized can citizens engage truthfully and productively.

Of course, there is a seeming tension between seeing the fact of these differences and identifying their corresponding value. It would seem that the thought of unbridgeable differences and impossible understanding would impede productive communication. And yet, I argue that these differences serve as a resource in sustaining the dialogical openness and attentive listening that deliberation requires. How can coming to terms with the limits to mutual understanding through language sustain greater openness in communication?

To answer this question, I will demonstrate how citizens are encouraged to reorient themselves towards others upon recognizing the limits to mutual understanding and the distance that (at least initially) divides them from each other. As I explain in the following section, when a citizen recognizes the deep differences that divide her from others, she may come to 1) view her beliefs as provisional, less secure, and particular. She also will likely 2) recognize her fellow citizens as worthy interlocutors bounded by the same limits as herself and therefore 3 ) delay her response, avoiding a premature dismissal, judgment, or cooptation of others' perspectives. This delayed response creates space in which to meet her interlocutors and actually consider what they say. Compared to empathy-based approaches, feelings of difference are a better resource for promoting dialogical openness precisely because of the provisionality, recognition, and delay in response that they produce. 
Heidegger's emphasis on the world-disclosing function of language and poetry can actually bring us closer to Habermas's goal of mutual understanding, thus supplementing (not supplanting) democratic deliberation. Recognizing the limits to communication and the unknowability of another has the potential to generate the humility, receptivity, and generosity of dialogical openness that is missing from existing models of democratic deliberation, including Habermas's. Therefore, we can best achieve Habermas's ethical-political ends of coordinating action and reaching democratic consensus by incorporating Heidegger's linguistic means.

\section{3.ii Humility and the Provisionality of Perspective}

As part of the perpetual concealment of the other in language, Heidegger explains, "what is known remains inexact, what is mastered insecure" $(1971,51)$. It is crucial that citizens recognize the inexactitude of their knowledge of others. Any mastery that we acquire over a subject or a decision is always insecure, tentative and provisional. Accepting the provisionality of this mastery helps to bring about dialogical openness.

According to Heidegger, accepting the provisionality of our understanding springs from the recognition of the perpetual distance between ourselves and others. Unless we hold our opinions to be provisional, or at least particular and contingent, there is no need for deliberation to occur. If we take our beliefs to be absolute or definitive, monologue will seem preferable to dialogue. Accepting the provisionality of our perspective therefore generates a certain level of humility among citizens as they see their understanding as incomplete and in need of improvement through engagement with others.

Furthermore, by recognizing the contingency of our own views and perspectives we see the epistemic value of our own situated knowledge. My life experiences and social context lead 
me to have a particular perspective that is unique and potentially valuable to my fellow citizens. The provisionality of my view generates both humility, in light of the fact that my perspective is not as universal as I originally suspected, and a corresponding curiosity to find out more about others' unique perspectives. Exposure to those who are different from myself reminds me that their views, perspectives and opinions are also likely to be relevant to our collective decisionmaking process. The views, perspectives, and opinions of others that I do not share can only be encountered through conversation with others.

Of course, encountering people with different perspectives who may, as a result, disagree with me could simply result in my dismissal of them as myopic, biased, or wrong. Applying democratic ideals to the literature on epistemic peer disagreement, however, leads me to a more optimistic understanding of the effect of disagreement on interlocutors.

As philosopher Thomas Kelly explains, "Suppose that, as it turns out, you and I disagree. From my perspective, of course, this means that you have misjudged the probative force of the evidence..." $(2005,179)$. According to Richard Foley, our disagreement defeats the prima facie reason I have to trust your opinion and deem you as equally suited at assessing question P (2001, 114). While disagreement among two individuals may lead them to believe that they are not, in fact, epistemic peers, in the context of democratic deliberation, they remain democratic peers. As a result, their differences in perspective are due equal consideration. Kelly's use of "from my perspective" is instructive here. From my perspective, the person who disagrees with me is wrong and therefore must have ignored or misinterpreted relevant evidence. But in terms of democratic deliberation, where equal consideration is a driving force, the difference in perspective is precisely what makes it valuable. Insofar as democracy requires equal 
consideration, we must hold all citizens as democratic peers, even if not epistemic peers. And in terms of including the variety of perspectives so as to increase the pool of social knowledge available to citizens making decisions, we cannot dismiss those who disagree with us. Their perspective is valuable by virtue of it being different. And it is the recognition of this difference that generates epistemic humility regarding our ability to know or imagine their perspective. Such humility makes us more attentive and more willing to listen to the actual perspective being expressed.

When we acknowledge the provisionality of our own perspectives, we come to see the situated (and therefore limited) nature of our own social knowledge. As a result, we recognize the other as having relevant and new information to bring to deliberation.

\section{3.iii Receptivity through the Recognition of the Other}

Heidegger's conception of language also gives us an idea of how two people can come to recognize each other as worthy interlocutors - a vital factor in generating the receptivity required for dialogical openness. For Heidegger, there is a sort of intimacy found in the perpetual conflict of lighting and concealing experienced by two adversaries brought into a "clearing" through language. He explains that the conflict between concealment and unconcealment "is not a rift (Riss) as a mere cleft is ripped open; rather it is the intimacy with which opponents belong to each other" $(1971,61)$. This intimacy of the opponents, insofar as they belong to each other, allows for recognition. The conflict or rift in Heidegger's conception “carries the opponents into the source of their unity by virtue of their common ground [...] this rift does not let the opponents break apart; it brings the opposition of measure and boundary into their common outline" $(1971,61)$. It is crucial to note here that the common ground Heidegger refers to need 
not be a substantive ethical agreement or life experience that could lead to empathy or a shared perspective. Rather, the common ground, or what is shared by interlocutors, is the space in the "clearing." What are shared are the limits of communication, expression, and understanding, the limits of unconcealing yourself and the fact of remaining forever concealed.

Encountering the differences and limits to mutual understanding between individuals helps remind citizens that we are all bounded by the same relationship to language as others. Though we do not all share the same facility with language or self-expression, we do share the same fundamental limits to complete understanding. By acknowledging our sharing of these limits, we come to recognize the other in her difference. Such recognition is a prerequisite for being receptive to what others have to say.

The recognition of the distance that exists between us and that which always remains concealed has the potential to foster recognition of one's fellow subjects. I say potential, however, because in the Heideggerian framework, recognizing the concealedness of the other does not necessarily guarantee a corresponding recognition of her subjectivity. Heidegger's attention to otherness in the clearing has a limited understanding of what it means to be other. As White explains, "Heidegger seldomly speaks of intersubjective otherness; and when he does, this form of otherness and human relationships is usually heavily subordinated to the question of otherness as it emerges within the question of being" (White 1991, 60). In other words, for Heidegger, respect for another being or entity does not assume respect for another's worth as a fellow subject.

I believe that despite this limited conception of 'otherness as being' and not necessarily 'otherness as subject,' Heidegger's notion of the perpetual concealedness of the other still allows 
for the development of a corresponding recognition for the other subject, qua subject. There is still recourse within his work to warrant our discussion of a Heideggerian intersubjectivity.

Heidegger asserts that being (or Dasein):

is equiprimordially being-with others and being-among entities encountered in the world. The world within which these latter beings are encountered is [...] always already a world that one shares with others. It is only because Dasein is antecedently constituted as being-in-the-world that one Dasein can existentially communicate something factically to another. (Heidegger 1982, 297, as cited by Kompridis 2006, 45. My emphasis).

Of course, "being-among entities" is not the same as "being-among subjects." Yet, insofar as Heidegger identifies this shared world as a world of communicators, he implies a sort of "beingamong other speakers," allowing for an intersubjective interpretation. Thus the dialogical openness that I seek to harvest from Heidegger's larger work has its roots in the perpetual, unavoidable concealedness present in language or speech acts specifically, and not existence more generally. Insofar as Heidegger identifies this concealedness within a linguistic framework, we can assume, at least on some level, another subject (read interlocutor).

\section{3.iv Generosity of a Delayed Response to Difference}

The recognition of difference encourages citizens to see the other as having relevant information to share. Through the inclusion of difference, the pool of knowledge is not only expanded, but we are shown the importance of drinking from its waters. When a speaker acknowledges both the illumination and concealedness present in her encounter with another subject, when she recognizes the limitations of communication and feels the deep difference that divides her from others, she comes to see the provisionality of her opinions and affords recognition to her interlocutor. These effects of feelings of difference lead to a third 
transformation wherein her assumptions, preconceptions, and prejudices are staved off (at least temporarily) and she delays in her response to what she sees as different and other.

In my acknowledgement of the conflict of lighting and concealing, I feel the deep differences that exist between myself and others. But it is not only the other who remains concealed; I remain concealed as well. Therefore, according to this understanding of Heidegger's “clearing," an individual cannot simply accept another's perpetual concealedness without also recognizing her own. Recognizing both your and my own difficulty with fully expressing ourselves has the potential to instill in us a certain level of patience and reserve. Such a realization of the limits of her own self-expression transforms the speaker, prompting her to afford a delayed response to alterity. Such a delay is required in order to create the space necessary for interlocutors to meet, to engage productively, and to show a generous consideration of alternative points of view.

\subsection{The Difference Approach Compared to the Empathy Approach}

Given the inevitability of difference and disagreement, democratic decisions can only be made if participants in discourse have a particular disposition - one of humility, receptivity, and generosity characterized by a deep openness to dialogue. I have shown how the recognition of differences that separate us from others can actually serve as a valuable resource for citizens cultivating this disposition.

With this account of the difference approach in mind, I want to conclude this chapter with a brief summary of how the difference approach distinguishes itself from the empathy approach. When compared to empathy, or Einfühlung, Heidegger's notion of Lichtung, or the lighting up 
that we experience in "a clearing" in a forest, provides helpful imagery in explaining the benefits of a difference approach to deliberation.

As Morrell (2010) notes, Edward B. Titchener originally coined the English word “empathy" from the German word Einfühlung, which means "feeling-into." Titchener took Einfühlung from the realm of aesthetics where the concept referred to the projection of one's own thoughts and feelings onto an inanimate aesthetic object. Titchener explained the concept with the image of a forest: "As we read about the forest, we may, as it were, become the explorer; we feel ourselves the gloom, the silence, the humidity, the oppression, the sense of lurking danger" $(1915,198)$. Becoming an explorer of the forest, we tread into dark corners, acquiring knowledge of this new and strange place. The intrepid explorer surveys, bravely throwing himself into the unknown. In contrast to the projection of Einfühlung, I propose an image more reticent and humble than Titchener's explorer.

Heidegger's concept of "the clearing" provides imagery to help explain the benefits of a difference approach to deliberation, one that highlights the limits to mutual understanding. When we are called into "the clearing" in a linguistic encounter, we do not run forward fearlessly to explore. Instead, Heidegger explains that we must recognize that which remains forever hidden or beyond our view. Remaining patiently in the clearing of the woods rather than bounding into the dark corners of the forest, we temper our urge to become masters of the forest. In Heidegger's clearing, we are more tentative and delayed in our exploration, as we are faced with the reality that some things will always remain shadowed and hidden from our view.

Deliberation is not improved by efforts to boldly overcome difference. Instead, greater space must be allowed for difference. An example of the power of the difference approach can 
be seen in the discourse following the death of Trayvon Martin. In the aftermath of Trayvon Martin's death, the phrase "I am Trayvon" was popularized in public discourse. People showed their condemnation of Martin's killing as well as the trial and acquittal of George Zimmerman. Ebony Magazine, for example, printed several covers featuring famous black men wearing hoodies and posing with their sons under the title "We Are Trayvon."

Growing out of this rallying cry, however, was a counter-movement of sorts that turned the original slogan on its head. The new rallying cry was: "I am NOT Trayvon Martin." This slogan has been used by white "allies" who, like many black Americans are disturbed by the outcome of the Zimmerman trial. Claiming "I am NOT Trayvon," however, demonstrates an admission on the part of these individuals that they cannot imagine or fully understand the experience of black Americans. It is not about the rhetoric per se, but the underlying disposition that one expresses by asserting, "I am Not Trayvon." Through this, speakers demonstrate humility, rejecting the presumption of fully understanding black Americans' perspectives on events surrounding the death of Trayvon Martin. This humility creates space wherein white Americans can listen to and hear the concerns and demands of black Americans.

Focusing on difference emphasizes the importance of listening, but more importantly, listening with humility, given the limits to our ability to ever fully understand. A realization of difference enables democratic citizens to potentially reconsider their moral or political commitments, to engage in meaningful evaluation, and begin to resist subtler aspects of power operating in political discourse.

Some might argue that expecting citizens to become more open in the face of deep differences is just as high a standard as the empathy approach that I have criticized. The potential 
for renewed closure in the face of differences is certainly a possibility. But while the difference approach may face the same challenges in practice, as a deliberative ideal it fares much better than the empathy approach.

Recognizing differences instead of commonalities maintains a theoretical focus on the challenges and limits to mutual understanding. The challenges to democratic discourse in a pluralistic world are significant, given that it occurs always already in the presence of disagreement. Given the magnitude of these challenges, it is crucial that citizens are aware of them. If these challenges are ignored in favor of an approach that focuses primarily on similarities and our ability to imagine and understand another's feelings and motivations, citizens are not alerted to the vital importance of engaging in rich, complex, and sometimes difficult listening practices. Empathy represents a proto-consensus that actually undermines the very possibility of some eventual agreement by diverting our energy from the need to actually enter into real discourse in the first place.

By emphasizing the problem of dialogical closure and the limits to mutual understanding, the difference approach cultivates the virtue of modesty in a citizen, helping her understand and realize the hard work of democratic decision-making and democratic listening. Even such a modest accomplishment is a marked improvement over the empathic approach.

\subsection{Conclusion}

Previous attempts to theorize how we might bring about a more open and receptive disposition on the part of citizen-listeners have focused on generating empathy. In contrast, I have explored how difference might be used to foster more inclusive democratic practices by alerting citizens to the urgent need for improved listening. My emphasis on the importance of 
difference in democracy fits within a line of theoretical inquiry that takes seriously the fact and value of difference. The difference approach that I propose, however, looks at the role that difference can play in improving listening and uptake in addition to pluralizing input. The question remains as to how such recognition of differences can be fostered so as to generate dialogical openness. If the recognition of difference can generate dialogical openness, how can citizens be made to recognize the differences that exist among democratic co-citizens? This question has interesting parallels to the question of how to generate empathy. Advocates of the empathy approach aim to demonstrate the value of empathy, but often fail to address ways that we may bring empathy about. In order to enhance the persuasiveness of my alternative to empathy, the next chapter examines aesthetic expression as one possible resource for bringing about dialogical openness through recognition of difference. 
Chapter 4. Aesthetic Sources of Dialogical Openness: Improving Deliberation Through Art Any theory of deliberation must attend to what I call "dialogical openness"- a disposition of greater humility, receptivity, and generosity on the part of citizens engaging in deliberation. Yet, as I explain in Chapter 1, this part of the puzzle is often ignored. In Chapter 2, I demonstrated the limits to an empathy approach to improving democratic deliberation and in Chapter 3 argued instead for greater attention to differences in order to bring about more capacious listening on the part of citizens. To continue with my argument in favor of a difference approach to sustaining practices of democratic deliberation, this chapter explores one possible way to enact the difference approach: encounters with aesthetic expression. Art can and often does foster empathy between individuals who, prior to an encounter with a novel, film, or piece of music, may not have recognized their commonalities. But an often-overlooked quality of aesthetics, and one that carries significant democratic value, is the way art can expose deep differences between ourselves and others. By highlighting differences rather than commonalities, I argue that some art can make us more attentive to the ways we remain closed off and unreceptive to others. What makes this exposure to difference so politically useful is the fact that it generally occurs in less intimidating and less fearful settings than straightforward political confrontations.

\subsection{A Theoretical Approach}

The approach I take to answering the question of how to generate dialogical openness among citizens is largely a theoretical one. As I argue in this section, the question I am asking cannot be proved conclusively through empirical research. That does not mean, however, that we should avoid speculating about the practices and efforts that might contribute to the cultivation of dialogical openness. 
Because it is more difficult to measure listening than speaking, empirically assessing the existence or effects of dialogical openness is methodologically tricky. Perhaps because of this, little attention has been paid to even theorizing possible sources of dialogical openness. For example, in Dissonance of Democracy - which is ostensibly the only book-length treatment of the importance of listening in deliberation - Susan Bickford ignores the question of cultivating better practices of listening until the last few pages. The question of how to cultivate a more open disposition is ignored, in part, because of its significant empirical implications. Similarly, after a few suggestions regarding the importance of public art and literature, Sharon Krause intentionally brackets the question of ways to cultivate greater receptivity and openness among citizens, claiming it is beyond the scope of her project. I argue it is precisely because of the empirical challenges to knowing or proving how and why aesthetic expression affects individuals that political theorists should pay careful attention to these sorts of questions.

Dialogical openness is not necessarily observable, but refers to a general sensibility among citizens. Dialogical openness defies observation, in part, because it is not expected to produce specific, consistent, predictable, or observable outcomes. Disagreement can remain as much between two dialogically open interlocutors as it can among speakers who are closed off. Similarly, I may eventually close myself off to what turns out to be hateful, racist, or unjust speech, even if I was initially open to listening. In contrast, I may appear to engage with others allowing them to speak - all the while refusing to actually consider what they are saying. Presidential debates are a perfect example of observable dialogue in the absence of meaningful openness between speakers. 
Observing an increase in dialogical openness remains a significant challenge to measuring or proving the link between certain practices and greater openness. As I explain in Chapter 3, this challenge is one possible explanation for the anemic investigation into the important question of improving listening and uptake in deliberation. Despite the steep challenges to quantifying or empirically proving these theoretical claims regarding how we might improve democratic discourse, the importance of listening to democratic outcomes demands this inquiry.

Interestingly, Goodin, Bickford, Krause, Morrell, Nussbaum, Young, all briefly suggest art, literature, film, and music as resources in deliberation, but none inquire into the precise connection between artistic expression and democratic discourse, let alone how art may help generate what I call dialogical openness.

This chapter explains the theoretical link between artistic expression and the cultivation of dialogical openness among citizen-listeners. I intend to draw a careful, cautious, and modest theoretical link between aesthetic expression and the enactment of the difference approach to generating dialogical openness. On the one hand, I want to go further than Goodin, Bickford, Krause, Morrell, and Young who merely gesture at the democratic potential of art in passing. On the other hand, I want to avoid the error that Simon Stow attributes to Nussbaum and Rorty who make claims that are too strong and inflexible regarding the effect of literature on citizens.

As Simon Stow demonstrates in Republic of Readers, Nussbaum's and Rorty's claims regarding what they see to be predictable and universal political effects of literature are too strong in light of the evidence that is actually available to support their claims. And yet I would argue that simply ignoring the question of how aesthetic expression can influence and improve 
democratic deliberation is no better than making exaggerated claims regarding its political power.

Ultimately, I argue that much of the democratic potential of aesthetic experiences lies in art's ability to disrupt one's standard view of the world, show a person the provisionality of her beliefs, and invite her to acknowledge the world that exists outside of herself. Aesthetic-affective expression in general, and not just certain works in particular (or even particular readings of certain works) have the potential to show a person that she can never fully understand or know the lives, experiences, and opinions of others. This realization can, in theory, make someone more receptive and generous to others and to difference, in part, because she realizes the limits to her own self-expression.

\subsection{Art and Politics}

The existing literature on aesthetics and politics is rich and varied. Much of the literature, however, can be placed into four broad categories, which I outline in detail in this section. ${ }^{15}$ The first camp focuses on the dangers of any aestheticization of politics. The second group embraces aesthetics as an alternative to the modern rationalistic understanding of politics, largely dismissing the deliberative project and its faith in human reason. A third category considers the adoption of aesthetic-affective modes of communication as an important end in itself, as one way to directly accommodate differences among citizens. The fourth, and most recent trend in this literature embraces art in politics for instrumental reasons, specifically for its promotion of empathy among citizens. In this chapter, I offer a fifth account of the relationship between art

\footnotetext{
${ }^{15}$ My classification of the literature on aesthetics and politics is not exhaustive, and the categories I present here are not mutually exclusive. I introduce these categories simply for clarity's sake and as a means of situating my contribution in the existing literature.
} 
and politics. Like the empathy camp, this account highlights the instrumental value of aesthetics in politics. But instead of focusing on the generation of feelings of commonality, I explore the ways that art may help us recognize and feel deep differences, with all the democratic benefits described above. Furthermore, instead of pointing to a consistent or predictable effect of aesthetic encounters (such as the promotion of empathy), my account focuses on the power and value of aesthetic expression precisely for the inconsistent and unpredictable effects that it can have on people.

I show that the historical exclusion of aesthetics from deliberation is theoretically unfounded and may be needlessly limiting the resources available to democratic theorists in our efforts to democratize deliberation through the cultivation of dialogical openness. To show how my difference-based approach to aesthetics and politics speaks to existing camps in the literature, I describe these categories in a bit more detail.

\section{2.i The Dangers of Aesthetics and the Promise of Reason}

Philosophers as early as Plato gave careful consideration to the implications - for Plato, the dangers - of allowing art to exist uncensored in the political realm. In Book X of his Republic, Plato banishes poets from his just city because poetry "seems to be a corruption of the minds of all listeners who do not possess as an antidote a knowledge of its real nature" (Republic 595b). Rather than revealing any sort of truth, of which poets are necessarily ignorant, poets will play to the peoples' appetitive natures and imitate "the thing that appears beautiful to the ignorant multitude" (Republic 602b). Importantly, art does not appeal to the deliberative or rational part of our soul, but instead to our appetitive natures. Poetry diminishes the justice of a polis by subverting our rationality. For this reason, poetry must be excluded from politics. 
Like Plato, Habermas prioritizes the use of reason over poetry in politics and has a deep distrust of incorporating aesthetic-affective elements into political discourse. In his Theory of Communicative Action, Habermas renders aesthetic expression secondary to rational communication. According to theorist Pieter Duvenage, “it is [Habermas's] concept of communicative reason, and its normative-critical role in the public sphere, that eventually direct aesthetics to a more marginal position in his work, thereby contributing to its fate" $(2003,22)$. Despite Habermas's “emphasis on intersubjectivity, he restricts the validity of art and aesthetic judgments to the subjective sphere of the author or creator" (Duvenage 2003, 97). To Habermas, aesthetics is an essentially expressive domain and therefore irreconcilable with the public sphere where we are called to justify our claims to others. In his model of democratic deliberation, aesthetic expression is distinct from, and less politically relevant than rational discourse.

Habermas, like his anti-democratic predecessor, prioritizes rational argumentation in his theory of communicative action. Argumentative speech overcomes participants' subjective views through the "mutuality of rationally motivated conviction" (Habermas 1984, 10). Intersubjective discourse is marked with a non-coercive logic or communicative rationality "from which democratic authority emerges" (Habermas 1996, 18). It is specifically a subject's reason that allows her to engage with others in collectively deciding how to coordinate action. Agreement is reached through the "central experience of the unconstrained, unifying, consensus-bringing force of argumentative speech" (Habermas 1984, 10). Habermas favors excluding aesthetic-expressive forms of communication from his model of democratic deliberation because such forms potentially undermine our rational capacities and eclipse what he takes to be the main function of language: solving problems and coordinating action. Such action coordination is the proper focus 
of politics and best achieved through rational discourse, not "world-disclosure," which is the focus of aesthetics.

Habermas's fear of incorporating art in politics runs parallel to the general wariness of liberalism to aestheticized politics. George Kateb (2008), for example, alerts us to the potential of aesthetic cravings to paper over serious threats to freedom and justice. Kateb defines aestheticism as "the effort to get from experience (let me clumsily call it non-art) what persons ordinarily seek and often find in works of art" (Kateb 2008, 12). The problem for liberal politics arises when morality and justice are sacrificed (often unintentionally) in pursuit of the beautiful or the sublime in everyday life.

As I am working within the Habermasian framework of discursive democracy, his rejection of aesthetics is of particular import. Instead of leaving his framework behind in favor of one more hospitable to the aesthetic enterprise, my goal is to defend the need for aesthetics in Habermas's own seemingly inhospitable theory. I aim to show why Habermas himself should be committed to incorporating aesthetic-affective communication into discursive politics, precisely for the priming role it can play in opening citizens up to the rational argumentation he favors so much. Rather than criticizing Habermas directly for his dismissal of aesthetics, my critique revolves around what I see as a deficiency of dialogical openness - the fact that Habermas does not adequately account for the disposition citizens need in order to engage in rational discourse in the first place. In order for people to have any chance of being motivated by the forceless force of the better argument, they must first be willing to listen carefully to that argument. In other words, they must be dialogically open. I turn to aesthetics as a resource for citizens cultivating such openness. 


\section{2.ii The Dangers of Reason and the Promise of Aesthetics}

Although recurrent throughout history, the negative view of art in politics from Plato to Habermas has not gone unchallenged. Aesthetics has arguably figured most prominently and positively in the political theory of Habermas's direct forbearers, namely the first generation of Frankfurt School thinkers. Marcuse (1955), for example, turned to art as the most likely source of liberation from the ills of a modern capitalist society and its hallmark faith in instrumental reason. Despite being the prime inheritor of the Frankfurt tradition, Habermas departed dramatically from his teachers in regards to aesthetics. Duvenage points to this paradox, explaining that although Horkheimer, Adorno, Marcuse, and Benjamim, "used aesthetically informed arguments in their respective socio-philosophical projects, this was much less the case with Habermas - the most prominent member of the second generation of Critical Theorists, who almost single-handedly steered this philosophical tradition in a new direction" - and decidedly away from aesthetics $(2003,1)$.

The appeal to aesthetics from the first generation of the Frankfurt School, however, was not totally abandoned in the study of political theory. Critics of Habermas, including Foucault and Derrida, who champion a politics that is more aesthetically infused, do not share Habermas's faith in human reason and the human capacity to reach consensus through "forceless" reasoned arguments. Showing a distinct hospitality to aesthetics as a means of critiquing modernity and its trademark trust in rationality, these French post-structuralists have in many ways carried the aesthetic torch of Habermas's teachers.

Like the French post-structuralists, the account that I give acknowledges the limits to human reason and our ability to justify our opinions in an exclusively "rational" way. Yet I do 
not abandon the deliberative ideal of linguistically mediated consensus altogether. In a novel way, I turn to aesthetics as a means to humble us in our dialogue with others, to show us the limits of mutual understanding. I give an account of why exposure to artistic expression may make us more attuned to the ways in which we are closed off and unwilling to listen to others. Such a realization enables democratic citizens to potentially reconsider their moral or political commitments, not by overcoming difference (as Habermas's critics would claim he does), but by affording greater space to such difference.

\section{2.iii Aesthetics as an End in Itself}

The third camp of scholars participating in the debate regarding aesthetics and politics includes those who call for pluralizing the means of deliberation to include more aestheticexpressive forms of communication. These scholars argue that Habermas's preference for rational argumentation is inherently exclusive and power-laden. Iris Young (1997B) has called for the inclusion of greeting, rhetoric, and story telling alongside more traditional forms of political communication (1997B, 70-72). Lynn Sanders (1997) has recommended testimony as a more democratic, less exclusive, form of communication compared to rational argumentation. I extend these calls for the pluralization of political communication, arguing for the incorporation of explicitly artistic forms of expression. Furthermore, though extremely sympathetic to these works, I aim to provide a theoretical account of how and why these and other alternatives to rational discourse contribute to the democratization of deliberation.

In their appeal for more aesthetic-expressive forms of communication, Young (1997B) and Sanders (1997) suggest that these forms of communication can help accommodate differences that exist in politics, and as a result, make deliberation more fair and inclusive. 
Accordingly, the pluralization of communication is thought to be an end in itself. It is a political goal, that if achieved could make deliberation more inclusive, at least at the margins. As I have shown in Chapter 1, however, missing from their arguments is a consideration of whether or why dominant groups would be any more likely to listen to or consider previously excluded opinions if they were voiced through alternative communicative means such as story telling or rhetoric. Making the procedures of deliberation more open to and inclusive of difference will not necessarily make the citizens themselves more open and attentive to difference. While it is important to empower voices by pluralizing the accepted forms of communication, attending to the attentive and receptive disposition on the part of listeners is at least as vital to ensuring fair and equal deliberation. No matter who is allowed to speak and in what manner, appeals made by marginalized groups are no more likely to be heard or considered until citizens adopt a more humble, receptive, and generous disposition. By focusing on the need for dialogical openness, I emphasize the importance of democratizing not only speech and expression, but also listening and reception.

\section{2.iv Aesthetics as a Means to Empathy}

More recently, those who admit the democratic value of aesthetics for instrumental reasons often tie that value to what they see as the empathy-inducing qualities of aesthetic expression (Goodin 2000, Krause 2008, Morrell 2010, Nussbaum 1995, Rorty 1989). Fostering a sense of empathy among citizens is perhaps the most intuitively plausible role for aestheticaffective expression to play in politics. Theorists who have made significant contributions to democratic theory by arguing precisely this include Danielle Allen (2004) who defends rhetorical and not "rational" speech as the basis for an improved view of democratic citizenship as 
friendship. Similarly, in discussing the need for "deliberation within," or empathic position taking, as a supplement to "deliberation without," Goodin gestures to aesthetics as an impetus to this process:

Suppose our imagination has been fired by some film or fiction; we have been led by those artifices to imagine vividly what it would be like to be they, or to be in that situation; we ask ourselves, 'What we would say, then?' $(2000,95)$.

For Goodin (2000), film and fiction have political value because they invite us to imagine and relate to the life of another and think differently because of it. Similarly, in Poetic Justice, Martha Nussbaum (1995) highlights the ways in which literature, especially the novel, allows citizen-readers to relate to characters who are very different from themselves. Sympathy is generated among readers of novels "with the thought that we ourselves might be in a character's position" (Nussbaum 1995, 91). Nussbaum explains what she sees as the potential of literary arts in politics, "Forming bonds of both sympathy and identification, they cause the reader or spectator to experience pity and fear for the hero's plight, fear, too, for themselves, insofar as their own possibilities are seen as similar to those of the hero" $(1995,53)$. For Nussbaum, literature acts on us to the extent that we can relate to the hero or imagine ourselves in her position.

Richard Rorty (1989) similarly looks to literature as a way of expanding the group of people "we" feel connected to and in solidarity with. Literature, according to Rorty, has political value in that it awakens us to the suffering of those to whom we could not previously relate. Allen, Goodin, Nussbaum, and Rorty all appear to be defending rhetoric, literature, and film as permissible forms of communication in politics because they can open citizens up to the concerns, suffering, and sacrifices of others. For all four, this opening is achieved by fostering 
feelings of sympathy or empathy between people, highlighting commonalities that exist between them.

Diana Boros (2012), who has recently argued for the power of art to improve civic engagement among citizens, identifies increased feelings of empathy as the mechanism bringing this engagement about. Boros makes two distinct but connected claims about empathy. First she claims that art is uniquely suited to promote feelings of empathy among citizens. Through works of art, we encounter universal existential truths about humans, bringing us closer to one another. Thus, Boros argues, works of art have the ability to induce feelings of empathy and togetherness among citizens. Second, Boros argues that these feelings of empathy are what will generate greater civic engagement among citizens. If we feel connected to each other, through empathy, we will be more likely to participate in the common project of politics.

As I discuss in Chapter 2, empathy can sometimes serve as a useful resource in democratic politics. Some events, such as the shooting at Newtown Elementary School, spark moments of national empathy that provide valuable political opportunities, as seen in the recent surge in popular support for stricter gun control laws. While it might be politically prudent to capitalize on these spontaneous moments of empathy, pursuing a politics of empathy as a means of opening citizens up to others has significant limitations.

Because of differences in experiences, perceptions, and imaginative capacities, we cannot assume that aesthetics will have uniform empathic effects on audiences. Although Nussbaum gives a convincing account of her experience reading Native Son, this cannot stand in as evidence of the power of literature or even this particular novel. In this chapter, I focus on ways that dialogical openness can be cultivated through art, such as a work of literature, without 
assuming a universal experience, reaction, or reading on the part of citizen-readers or citizenspectators.

As Stow argues, Nussbaum and Rorty's claim that "particular books will have definite and predictable impacts upon their readers is an impossible one to maintain" $(2007,121)$. By pointing to a precise effect that texts have on readers, Nussbaum and Rorty end up focusing on the text more than the readers themselves (Stow 2007, 121). Furthermore, championing their own particular readings rather than letting the texts speak for themselves "suggests that they too

have less than complete faith in this aspect of their claims," specifically the supposedly universal effect that certain works will have on citizens (Stow 2007, 121).

In contrast to these theorists, I argue that aesthetic-affective expression, including literature, is valuable precisely because of the variable and multiplicitous effects and readings it will produce among citizens. The democratic value of aesthetic-affective expression lies in the way it highlights difference and contingency "out of the multiplicity of possible textual readings" (Stow 2007, 135). It is exactly the multiplicity of possible textual readings that Nussbaum and Rorty ignore in favor of their preferred reading. In line with Stow, the difference approach as enacted through aesthetic expression offers a renewed emphasis on readers over texts, listeners over speech, and audiences over works. Rather than offering one particular interpretation of a work of art, I introduce art as well as its variable and conflicting interpretations as a valuable addition to democratic discourse,

\subsection{Aesthetics and the Cultivation of Dialogical Openness}

I identify three specific qualities of art that can help citizens cultivate the dialogically open disposition that most theories of democratic deliberation simply assume. First, the 
celebrated multiplicity of artistic forms and interpretation can encourage citizens to accept the provisionality of their beliefs, humbling them in their encounters with others. Second, the disruptive effect of aesthetic expression - part of what causes the enjoyment and delight of our encounters with art - can foster greater receptivity among citizens who come to recognize each other as worthy interlocutors. Lastly, the ethos of a delayed response that is encouraged in encounters with art make such encounters fertile ground for cultivating generosity on the part of citizen-listeners. These three aspects of aesthetics, lead me to conclude that aesthetic communication has much to contribute to democratic discourse.

\section{3.i Multiplicity and the Provisionality of Beliefs}

The realm of aesthetic judgment and tastes differs significantly from the realm of political judgment, traditionally understood. According to Kennan Ferguson, "aesthetic forms that can be read in multiplicitous ways are usually seen as richer, deeper, and finer than those which are transparently singular" (1999, viii). Because of the celebrated multiplicity of aesthetic taste, aesthetic contention is "more likely to resist totalized claims" (Ferguson 1999, viii). Harnessing the effects of this multiplicity might encourage citizens to adopt a political posture more accepting of the provisionality of their beliefs.

Valuing such a multiplicity of interpretations is relatively unique to aesthetics. In social sciences, for example, if two theories produce operationally equivalent predictions or if a collection of data can be explained by two distinct theories, then something is thought to have gone horribly wrong. Such ambiguous theories would be considered worthless in fostering a greater understanding of the world. Similarly, in the context of, say, the Supreme Court of the U.S., the admission of multiple, valid interpretations of a single law is not permitted, and perhaps 
could never be. The adversarial nature of the law encourages black and white interpretations, intended to enhance clarity and produce definitive answers. When it comes to democratic deliberation, however, in order for citizens to listen to and consider alternative viewpoints or perspectives, they must accept a certain level of provisionality in their own beliefs and preferences. The need for and value of provisionality is especially strong in informal and cool deliberative settings that have been decoupled from formal decisions making bodies.

The multiplicitous nature of aesthetic forms, judgment, and tastes distinguishes it thoroughly from rational, political discourse traditionally understood, making it a prime candidate for fostering the dialogical openness required for deliberation that is non-totalizing and hospitable to plurality and difference. We assume limitations in our capacity to completely understand or justify our aesthetic tastes and feel no obligation to settle all disagreements. In the realm of aesthetics, the limits to mutual understanding are well known and even celebrated. The epistemological humility that goes along with the recognition of these limits is precisely what prevents the formation of totalizing claims. People can remain open to others' tastes and invite differences in opinions while also remaining committed to their own provisional beliefs. Furthermore, aesthetic tastes are not totally subjective, as Habermas claims. Aesthetic judgments are often developed in precisely the intersubjective context that Habermas describes. People justify and persuade others of their own aesthetic preferences. Importantly, however, this justification and persuasion is more likely to resist the need for a final resolution or reconciliation of ideas and perspectives. The value placed on maintaining multiple, different, and competing tastes in aesthetic forms encourages people to accept and celebrate the provisionality of tastes and preferences. 
As with the realm of aesthetics, I argue that provisional beliefs in politics are to be commended and are not to be confused with relative beliefs. Provisional beliefs allow for epistemic commitment and humility to exist side-by-side. In Plato's Crito, Socrates defends his decision to die rather than flee Athens. Socrates, who claims to know only his own ignorance, does not believe he can be absolutely certain of anything, including the justice of staying in prison. Instead he can only cite provisional beliefs for this decision, beliefs that would be changed if other information came to light. Socrates' epistemic humility prevents him from asserting any absolute truths or pure and uncontestable reasons. Even after deliberating and reaching a decision, all he can assert is that accepting his death is the best course of action for now (Crito 46b). Although absolutely committed to his judgment and willing to die for it, Socrates never denies its inherent provisionality.

Socrates, of course, is a unique case. I do not want to suggest that all people could or should adopt such an extreme provisional orientation to their own beliefs and convictions. And yet this admission of provisionality mirrors the kind of stance that people often take in regards to questions of aesthetic judgment. Cultivating a form of provisionality in politics through the incorporation of artistic expression has the potential to humble us, opening us up to further discussion and to the possibility that we are wrong (in either our opinions, our understanding of the issue at a more basic level, or our relationship to the democratic community). Such an orientation to politics allows us to potentially change our minds, transform our will, or reconsider our commitment to a contentious policy or preference.

\section{3.ii Reception and Recognition through Disruption}


In order to ensure that people consider and listen to the views of others, they must first be drawn out of their own perspectives and lived experiences. Art helps bring this about by disrupting or, perhaps more accurately, interrupting a person's standard view of the world. By disrupting a person's view of the world, art has the ability to induce the recognition of the outside world, and more importantly those who occupy it.

The enjoyment and delight that audience members experience when encountering works of art come, in part, from the disruptive effect that aesthetic encounters can have. In her manifesto, The Uses of Literature, Rita Felski (2008) highlights the power of literature to depict discontinuities with everyday experiences. She writes, "The literary work enables an encounter with the extraordinary, an imagining of the impossible, and openness to pure otherness, that is equipped with momentous political implications" $(2008,5)$. But even in "those elements of familiarity, generic commonality, even predictability that shape, however subtly, all literary texts," readers are still drawn out of their own experiences (Felski 2008, 5-6). No matter how much I can relate to a particular novel, in reading the artful description, the other interjects, giving her own interpretation of the mundane as well as the extraordinary. By interrupting a person's relatively comfortable orientation to the world, even if not contradicting it, art serves as a resource in deliberation by encouraging citizens to recognize even just the existence of others. This recognition is crucial for successful deliberation to occur. Accounting for one possible source of such recognition marks an important improvement to Habermas's view of communication.

Art, in its form rather than its particular content or interpretation, has a way dislocating us from what may be a comfortable but unchallenged worldview. Such a disruption draws us out of 
ourselves, encouraging us to see and to recognize the world around us. In literature, for example, although reading is often a solitary action, we are necessarily, though perhaps superficially, drawn out of our own thoughts and ideas and are able to entertain and serve as temporary host to the thoughts and ideas of another. Ironically, this is not necessarily the case when we engage in actual dialogue with another person. Rational discourse or debate between two people may appear to serve the same disruptive function, and ideally should draw people out of their own thoughts. But the attention that we give to another in reading literature is inherent and unavoidable. We cannot read without internalizing (if only for a fleeting moment) another's words. But we can and often do hear without listening, without investing any of our own energy in understanding the other's thoughts or ideas. In a conversation with you, my attention is not automatic. In the act of reading a novel, however, I necessarily donate my own voice and attention to the thoughts of another. And I would argue that this is not just a function of reading. Reading another's rational arguments also fails to necessarily draw one out of his own mind. It is the imaginative, pleasurable, and delightful aspects of literature that immerse us in another's story and ensure this essentially disruptive effect. The disruption of our own monologue which occurs in reading fiction and ideally would occur in real-life dialogue has the potential to bring differences to our attention while also encouraging the recognition of those who embody those differences. This facilitates the cultivation of receptivity, one of the key components of a dialogically open disposition.

By interrupting my view of the world, art draws me out of myself. But this drawing out of myself must not be coupled with assuming the point of view of another. Unlike empathic approaches to politics, which advocate the recognition of oneself in another, the recognition that 
I believe art can generate comes from acknowledging someone as different, distinct, and wholly other to one's own self. Once I am drawn out of myself, I begin to recognize others, yet they remain distinct from myself. The value of artistic expression in democratic deliberation is simply the fact that it helps me to recognize the existence of the other and accept that they may have a view different from my own. But the recognition or acknowledgement that disruption can bring about must not be confused with a total reconciliation (Felski 2008, 31).

Importantly, the distance between the reader and the character inherent in the act of reading literature ensures that recognition does not devolve into cooptation. This distance is crucial. In art, the distance between your world and your world as you construct it for me is unavoidable and undeniable (Bickford 1996, 147). My knowledge of your life is always mediated, and art renders this mediation more visible, drawing our attention to the intersubjective nature of our world as well as the limits to mutual understanding. Knowing these limits is a first step toward the dialogical openness required for deliberation.

For example, the potential of the novel to dislocate the reader from her own life and frame of reference, situating her instead within the life of the novel, generates at least a superficial opening. Readers do not actually come to know or experience another's life, as such, but instead engage with an alternative depiction of the world that has the potential to challenge their own. Reading novels helps “to slightly dislocate oneself from one’s settled identity as host and center" (White 2008, ix-x). This dislocation makes readers more receptive to difference as they call into question what was previously assumed and settled.

At first glance, it may appear that the disruption that I am calling for might actually lead to a "closing off" rather than an "opening up." Intuitively, we might assume that feelings of 
disorientation, anxiety, and confusion would lead to less and not more receptivity. Despite this intuition, empirical evidence actually points in the opposite direction. Studies in political psychology find that anxiety actually promotes deliberation and reconsideration - though not always with democratic results - whereas comfort and continuity encourage people to maintain their prior assumptions (Marcus 2002). As Ted Brader explains in a 2006 review of the empirical literature on emotions in politics, "there is a good deal of evidence that anxiety can provoke attention and information seeking...motivate action... and precipitate a change of mind based more on contemporary information and less on predispositions..."16

\section{3.iii Delayed Response, the Sublime, and Generosity}

A third way that aesthetic expression can enact the difference approach to cultivating dialogical openness has to do with its ability to bring about a delayed response. Art appreciation often requires, but more importantly encourages, a "delayed response," as opposed to a quick or thoughtless reaction. Citizens engaged in political discourse would benefit from cultivating the ethos of delayed response that is often afforded to artistic expression. The world-disclosure brought about in a work of art is not always automatically accessible; art appreciation requires people give careful consideration to what remains "concealed," "unknowable," or "unseen." And this consideration accounts for much of the pleasure, delight, but also frustration we feel in our encounters with a work of art. Recognizing the limits to their own understanding, spectators delay judgment. Although caught in the conflict of concealment and unconcealment as discussed in Chatper 3, aesthetic forms, as such, still invite us to engage with the world outside of

\footnotetext{
${ }^{16}$ Brader points to the following sources: Brader 2006; Brader, Valentino, Suhay 2004; Feldman, Huddy, N.d; Marcus, Neuman, MacKuen 2000; Redlawsk, Civettini, Lau 2005; Valentino, Hutchings, Banks, Davis 2006; Rahn, Kroeger, Kite 1996; Valentino, Hutchings, Gregorowicz, Groenendyk 2006; Kiner, D’Ambrosio 2000; Way, Masters 1996.
} 
ourselves. This engagement, however, is one of greater generosity as we come to see our own limits and therefore act more generously when confronted with the limits of others.

Edmund Burke's concept of the sublime is helpful in understanding the effect that certain works of art can have on us, especially the effect of encouraging a delayed response. In $A$ Philosophical Enquiry into the Origin of Our Ideas of the Sublime and Beautiful, Edmund Burke explains that the sublime is that "which excites ideas of pain and danger," whereas the beautiful is associated with pleasure (1909 [1757], 36). The sublime relates to fear when felt in the face of pain and danger that we can "enjoy" from a distance. In the face of that which is sublime, we feel "delight" according to Burke. "Delight" is a kind of muted pleasure that occurs only when the threat of pain is mediated and relatively distant. But Burke argues that delight is not merely a matter of taking pleasure in the distance itself. He claims that delight relates to a pleasure in the painful observation. If the threat is too close to us, however, we feel no delight and instead are simply terrified.

I engage with Burke's aesthetic theory because of the ways he ties the encounters with the sublime together with feelings of human limitedness. According to Stephen White, "Burke is telling us that [the sublime's] deepest significance as a category of human experience resides in the way it confronts us with our finitude" $(1994,30)$. Famously, Kant also distinguishes between the beautiful and the sublime. Though somewhat similar in their understandings of the sublime, Burke's account takes us further than Kant's depiction, offering a slightly more expansive view of the sublime. In his refiguring of the sublime, Kant describes the sublime as an affective state wherein humans are able to marvel at their participation in the infinite, in the noumenal realm 
(White 1994, 6). Kant focuses on the limitlessness that we feel when we are elevated beyond the physical world in "comprehend[ing] the incomprehensible" (Eagleton 1990, 74).

For Kant, encounters with the sublime actually represent a celebration of the limitlessness of our mind and our capacity for reason rather than our limitedness. Burke, however, helpfully highlights the anxiety that humans feel in that moment of incomprehensibility. Recognizing that comprehending the incomprehensible and knowing our own limits comes with some anxiety, Burke offers a more accurate and complete depiction of the sublime than Kant. For Burke, encounters with the sublime allow us to confront our finitude, our own death.

This confrontation "is neither merely a cognitive pondering nor an emotional reaction, but rather an experience in which cognition and passion are inextricably bound up with one another" $(1994,30)$. The sublime's ability to work on us in both a cognitive and emotional way make it an ideal resource in cultivating the dialogical openness I claim is necessary for deliberation to succeed.

My claim regarding how feelings of difference between ourselves and others can make us more generous in our encounters with our fellow citizens can be interpreted as an application of Burke's idea of the sublime as a reminder of our "limitedness." In a political context, encounters with the sublime through art allow citizens to experimentally confront those who are different from themselves. In this hypothetical confrontation, a spectator or reader recognizes she can neither fully understand another's perspective nor, importantly, can she fully share her own perspective with another. As I discuss in my development of the difference approach, recognizing both your and my own difficulty with fully expressing ourselves has the potential to 
instill in us a certain level of patience and reserve, creating the temporal space needed for interlocutors to meet, to engage productively, and to consider alternative viewpoints.

We are limited in how we can relate to our fellow citizens, limited in how we can express ourselves, and limited in our ability to come to a mutual understanding with others. Recognizing this limitedness, the impediments to mutual understanding in the face of deep, constitutive differences, through encounters with art, especially art that is sublime, can bring about the delayed response needed to make citizen-listeners more generous in dialogue. Drawing our attention to the limits of mutual understanding and communication, art discourages a quick or thoughtless reaction, and thus helps us avoid the premature dismissal, judgment, or cooptation of others' perspectives. This delay is an invaluable feature for politics.

Even art that may not fit into Burke's category of the sublime still has the potential to encourage a delayed response on the part of spectators. The disruptive effect that art can have on audiences together with the celebrated multiplicity of aesthetic form and taste induce a relative delay in response from spectators who become attentive to the limits in their own perspective and understanding. Such a practice of delayed response would be an invaluable feature for politics.

\subsection{Conclusion}

Aesthetic expression is a valuable resource that can help citizens cultivate greater humility, receptivity, and generosity in their dialogical encounters with fellow citizens. The potential for a work of art to generate dialogical openness lies in its ability to highlight deep differences between citizens, thereby inducing citizens to delay in their response to others, to accept the provisionality of their beliefs, and to recognize the worth and distinctiveness of their 
interlocutors. The features of aesthetics that I discuss in this chapter, together with their variable effects, can help bring about a transformation of citizens' disposition towards difference.

Citizens who adopt this more dialogically open orientation are better able to fulfill the demands of listening in democratic deliberation. 


\section{Conclusion}

In this dissertation I have searched for new answers to the problem of political inequality and exclusion in democratic theory. The intervention that my dissertation makes into democratic theory is best understood as a complement or supplement to existing accounts of democratic deliberation. I have not offered my own theory of democratic deliberation, but instead have aimed to address and clarify oversights and missing links in existing theories.

To that end, a major goal of this dissertation has been to develop a more complete understanding of the concept of inclusion to be used in deliberative models of democracy. Inclusion or the equal consideration of all citizens is at the heart of the promise of democracy. And as I have shown in this dissertation, inclusion is dualistic in nature. The duality of the democratic ideal of inclusion can be seen in a simple example of voting. In a purely aggregative model of democracy, where citizens' preferences are registered primarily through voting (rather than discourse), citizens are included - in short, allowed to vote - with the fundamental assumption that all votes will be counted equally. Equal consideration obviously requires not only that citizens be allowed to cast a vote, but also requires that their vote be counted. A system of democracy that allowed citizens to vote without guaranteeing each vote would be counted would be no democracy at all. Similarly contradictory, is a deliberative model of democracy that allows all individuals to speak without a corresponding promise to listen to what they have to say.

The deliberative ideal requires more than this singular understanding of inclusion as access. In fact, I would argue that the struggle for inclusion only truly begins in earnest once access has been gained. This struggle for inclusion by way of equal consideration and not just equal voice has been the topic of this dissertation. In order to address this often-overlooked 
aspect of inclusion I have introduced the concept of dialogical openness. In this dissertation I search for resources to help citizens cultivate a disposition of greater dialogical openness and its hallmark humility, receptivity, and generosity. To begin, I considered recent claims that empathy helps promote greater inclusion in democratic discourse.

In Chapter 2, I provided reasons to be skeptical about recent claims that the renewal of our American democracy hinges on an increase in empathy. I argued that when introduced into models of democratic deliberation, empathy defined as both imagined perspective taking and empathic concern fails to sustain deliberation. Although empathy may promote greater reflection or deliberate-ness, it does so at the expense of deliberative-ness, or actual communication. If you can imagine how your fellow citizens feel, the need to actually listen to particular others is no longer as urgent. Therefore, empathy does not necessarily promote the robust understanding of inclusion described above.

The difference approach that I propose as an alternative to the empathy approach generates dialogical openness through feelings of difference rather than feelings of real or imagined commonalities. I have tried to show the ways in which feeling the differences between ourselves and others can dampen our presumption of knowing the other person's perspective. But what exactly is the value of dampening this presumption? I admit that neither the empathy approach nor my own difference approach offers a silver bullet in regards to guaranteeing dialogical openness. But by taking seriously the problem of dialogical closure and the limits to mutual understanding, the difference approach emphasizes the hard work of democratic listening and decision-making. For these reasons, I argue that it is better suited to the task of making deliberation more inclusive. 
In the final chapter, I turned my efforts to identifying one possible resource in generating dialogical openness through the difference approach. Here, I made the case for including artistic expression in models of deliberation. This proposal marks my biggest departure from Habermas's model of deliberation, which favors rational argumentation over more aestheticized forms of communication.

Again, a central claim of this dissertation has been that, by itself, increasing the perspectives that are expressed in deliberation is not enough to ensure inclusion. In addition to being permitted, these perspectives need to be critically engaged and carefully considered. Similarly, I have argued that the internal reflection that occurs within individual citizens is no substitute for the intersubjective deliberation among citizens. The same may be said about my argument regarding the inclusion of aesthetic expression. It is not enough that citizens encounter a work of art, whether by reading a book, watching a film, or going to a museum. They must also be called to reflect collectively on that work. To achieve the benefits of dialogical openness that I have speculated art can bring about, citizens must be encouraged to talk about the works of art that they encounter.

If art can serve as a resource in bringing about more dialogical openness, how then do we get citizens to be open to art and especially conversations about art in the first place? Although in Chapter 2, I criticize Morrell's vague calls for “empathy education," I am not generally opposed to civic education as a potential solution to the problem of dialogical closure. My concern is not so much with his reliance on education, but with his belief that civic education should be directed at promoting empathy. In my view, the content of this education must not try to reduce all individuals - by virtue of our common humanity - into one empathetic melting pot. 
Instead, I would endorse an approach to civic education that highlights the extraordinary range of human possibility that exists in each unique life and culture. Though necessarily vague in its brevity, I propose arts education as one possible subject and focus of the civic education that Morrell advocates.

The first empirical study ever to demonstrate a causal relationship between arts education and desirable intellectual and emotional effects was published in January of this year (Greene et al. 2014). Before this 2014 study, evidence for claims regarding the benefits of arts education did "little more than establish correlations between exposure to arts and certain outcomes" (Risida et al. 2013). But over the past couple of years, social scientists Brian Kisida, Jay P. Greene, and Daniel H. Bowen conducted a natural experiment coinciding with the opening of the Crystal Bridges Museum of American Art in Bentonville, Arkansas. "Through a large-scale, randomassignment study of school tours to the museum, [they] were able to determine that strong causal relationships do in fact exist between arts education and a range of desirable outcomes" (Kisida 2013). As a result of a single museum visit, students developed stronger critical thinking skills, higher levels of social tolerance, and a newfound taste for art museums and cultural institutions. In the future, I hope to engage further with this cutting edge research.

These initial empirical findings support my speculations that exposure to aesthetic expression can have important democratic potential. Renewed attention paid to the democratic value of arts education is warranted more than ever in light of recent cuts to arts education since the Federal No Child Left Behind Act and the emphasis it placed on testing in "core" subjects (Bowen et al., 2013). 
I want to be careful not to suggest that it is by virtue of simply being exposed to artistic expression that citizens will become more dialogically open. Instead dialogical openness may result from our incorporating aesthetic expression into models of deliberation so that they can be discussed and interpreted collectively. Stow's conclusion about literature seems to apply to my claims regarding the power of aesthetic expression more generally: "Literature alone will not lift readers out of solipsism but conversations about literature might” $(2007,124)$. For this reason, I believe arts education can play an important role in contextualizing citizens' encounters with art. Arts education would not only help ensure greater citizen exposure to art, but would also ensure that this exposure occurred alongside conversations among students, their teachers, and their families.

Rather than suggesting that museum visits or engagement with particular works of art will have predictable and universal effects on those who encounter them, I try to develop an understanding of the value of art that comes precisely from the variable, multiplicitous, and unpredictable effects it can have on citizens. In my view, the democratic value of art is not its ability to lead us to particular conclusions. I do not believe that reading certain works of art will teach citizens to shun cruelty as Rorty suggests, or help privileged white Americans identify with a poor black boy growing up in Chicago's South Side as Nussbaum claims. These sorts of claims simply reduce aesthetic expression to rational argumentation in a different form. I value aesthetic expression precisely for its ability to disrupt this attempt to reach complete understanding of another person. Art and conversations about art are valuable insofar as they give us pause and show us that the other evades easy understanding. We cannot place ourselves in another person's shoes as easily as we may think. And realizing this has democratic potential insofar as it alerts us 
to the hard work of democratic discourse in the presence of difference. I turn to art not so that it can lead us to particular conclusions, but because of its potential to draw us into conversation in the first place. 


\section{Bibliography}

Abizadeh, Arash. 2007. "On the philosophy/rhetoric binaries: Or, is Habermasian discourse motivationally impotent.” Philosophy Social Criticism, 33: 4, pp. 445-472.

Ackerman \& Fishkin, 2002. “Deliberation day.” Journal of Political Philosophy 10, pp. 129-52.

Allen, Danielle. 2004. Talking to Strangers: Anxieties of Citizenship since Brown v. Board of Education. Chicago: University of Chicago Press.

Benhabib, Seyla, ed. 1996. Democracy \& Difference. Princeton, NJ: Princeton University Press.

Bessette, Joseph M. 1980. "Deliberative Democracy: The Majority Principle in Government.” In How Democratic is the Constitution?, Robert Goldwin and William Shambra. Washington, D.C.: American Enterprise Institute, pp. 102-116.

Bickford, Susan. 1996. The Dissonance of Democracy: Listening, Conflict, and Citizenship. Ithaca: Cornell University Press.

Bohman, James. 1996. Public Deliberation: Pluralism, Complexity, and Democracy. Cambridge, MA.: MIT Press.

---------. 2004. "Realizing deliberative democracy as a mode of inquiry: Pragmatism, social facts, and normative theory." Journal of Speculative Philosophy 18, no. 1.

Bohman and Rehg, eds., 1997. Deliberative Democracy: Essays on Reason and Politics. Cambridge, MA: MIT Press.

Boros, Diana. 2012. Creative Rebellion for the Twenty-First Century: The Importance of Public and Interactive Art to Political Life in America. New York: Palgrave Macmillan.

Bowen, Daniel H., Jay P. Greene, Brian Kisida. 2014. "Learning to Think Critically: A Visual Art Experiment." Educational Researcher, 43: 1, pp. 37-44.

Brader, Ted. 2006. "Affective Intelligence and Beyond: Next Steps in Research on Emotion in Politics." Political Communication Report, 16: 3.

Burke, Edmund. 1909 [1757]. On the Sublime and Beautiful. New York: PF Collier \& Son.

Button, Mark. 2005. “A Monkish Kind of Virtue?” For and against Humility.” Political Theory 33: 6, pp. 840-868.

Cohen, Joshua. 1989. "Deliberation and Democratic Legitimacy." In The Good Polity Normative Analysis of the State, Alan Hamlin and Phillip Petit, eds. New York: Blackwell. 
Coles, Romand. 1997. Rethinking Generosity: Critical Theory and the Politics of Caritas. Ithaca, NY: Cornell University Press.

Connolly, William. 1995. The Ethos of Pluralization. Minneapolis: University of Minnesota Press.

Dalhberg, Lincoln. 2005. "The Habermasian Public Sphere: Taking Difference Seriously?" Theory and Society 34: pp. 111-136.

Davis, Mark H. 1994. Empathy: A Social Psychological Approach. Madison, WI: Brown \& Benchmark Publishers.

Dewey, John. 1988 [1927]. The Public and its Problems. Athens OH: Ohio University Press.

Dryzek, John. 2002. Deliberative Democracy and Beyond: Liberals, Critics, Contestations. New York: Oxford University Press.

--------. 2005. "Deliberative Democracy in Divided Societies: Alternatives to Agonism and Analgesia" Political Theory, Vol. 33, No. 218.

Duvenage, Pieter. 2003. Habermas and Aesthetics: The Limits of Communicative Reason. Cambridge: Polity Press.

Eagleton, Terry. 1990. The Ideology of the Aesthetic. Oxford: Blackwell Publishing Ltd.

Estlund, David. 2009. Democratic Authority: A Philosophical Framework. Princeton, NJ: Princeton University Press.

Fast, Omer, The Casting (Centre Pompidou, 2008).

Felski, Rita. 2008. The Uses of Literature. Malden, MA: Blackwell Publishing Ltd.

Ferguson, Kennan. 1999. The Politics of Judgment: Aesthetics, Identity, and Political Theory. Lanham, MD: Lexington Books.

Foley, Richard. 2001. Intellectual Trust in Oneself and Others. New York: Cambridge University Press.

Frazer, Michael. 2010. Enlightenment of Sympathy. Oxford: Oxford University Press.

Goodin, Robert E. 2000. "Democratic Deliberation Within.” Philosophy and Public Affairs, 29: 1, pp. 81-109.

--------. 2003. Reflective Democracy. Oxford: Oxford University Press. 
Goodin, Robert E. and Simon J. Niemeyer. 2003. Political Studies 51: 4, pp. 627-649.

Greene, Jay P., Brian Kisida, and Daniel H. Bowen. 2014. "The Educational Value of Field Trips." Education Next, 14: 1.

Gutmann and Thompson, 1996. Democracy and Disagreement. Cambridge, MA: Harvard University Press.

Habermas, Jürgen. 1984. The Theory of Communicative Action, Volumes I and II. Trans. Thomas McCarthy. Boston: Beacon Press.

--------. 1991. Moral Consciousness and Communicative Action, trans. Christian Lenhardt and Shierry Weber Nicolsen. Cambridge, Mass.: MIT Press.

--------. 1994. “Three Normative Models of Democracy.” Constellations 1 (1): 1-10.

-------. 1996. Between Facts and Norms: Contributions to a Discourse Theory of Law and Democracy. Trans. William Rehg. Cambridgem, MA: MIT Press.

Heidegger, Martin. 1971 [2001]. "The Origin of the Work of Art.” In Poetry, Language, Thought. New York: Harper Perennial Modern Classics.

-------. 1977. The Essay Concerning Technology. New York: Garland Publishing.

------. 1980. Holderins Hymnen “Germanien” und “Der Rhein,” Vol. 39, Gesamtausgabe. Frankfurt: Klostermann.

--------. 1982. The Basic Problems of Phenomenology. Bloomington, IN: Indiana Univ. Press.

Hoffman Martin L. 2011. "Empathy, Justice, and the Law." In Empathy: Philosophical and Psychological Perspectives, eds. Amy Coplan and Peter Goldie. Oxford: Oxford University Press.

Hume, David. 1968. A Treatise of Human Nature, ed. L.A. Selby-Bigge. Oxford: Clarendon Press).

Kant, 1987. Critique of Judgment. Indianapolis, IN: Hackett.

Kateb, George. 2000. "Aestheticism and Morality: Their Cooperation and Hostility." Political Theory, 28:1, pp. 5-37.

Kelly, Thomas. 2005. "The Epistemic Significance of Disagreement.” Oxford Studies in Epistemology, 1: pp. 167-196. 
Kisida, Brian, Jay P. Greene, and Daniel H. Bowen. 2013. “Art Makes you Smart.” New York Times. November 23, 2013.

Kompridis, Nikolas. 2006. Critique and Disclosure Critical Theory between Past and Future.

Krause, Sharon R. 2008. Civil Passions: Moral Sentiment and Democratic Deliberation. Princeton: Princeton University Press.

Krause, Sharon R. 2010. "Empathy, Democratic Politics, and the Impartial Juror." Law, Culture and the Humanities 7: 1, pp. 81-100.

Krauthammer, Charles. 2012. “Same-sex Marriage Empathy or Right?” Washington Post. May $17,2012$.

Lithwick, Dahlia. 2012. “It’s About the Empathy, Stupid.” Slate. May 10, 2012.

Love, Nancy. 2006. Musical Democracy. Albany: State University of New York Press.

Manin, Bernard. 1987. “On Legitimacy and Political Deliberation.” Political Theory, 15, pp. 338-368.

Mansbridge, J., Bohman, J., Chambers, S., Estlund, D., Føllesdal, A., Fung, A., Lafont, C., Manin, B. and Martí, J. 1. 2010. "The Place of Self-Interest and the Role of Power in Deliberative Democracy.” Journal of Political Philosophy, 18, pp. 64-100.

Marcus, George E. 2002. The Sentimental Citizen. University Park: Pennsylvania State University Press.

-------. 2013. “Reason, Passion, and Democratic Politics.” In Nomos LIII: Passions and Emotions, ed. James E. Fleming. New York: NYU Press.

Marcuse, Herbert. 1955. Eros and Civilization. Boston: Beacon Press.

McCarthy, Thomas. 1994. "Kantian Constructivism and Reconstructivism: Rawls and Habermas in Dialogue." Ethics: 105, pp. 44-63.

Morrell, Michael E. 2007. "Empathy and Democratic Education.” Public Affairs Quarterly 21: 4, pp. 381-403.

--------. 2010. Empathy and Democracy: Feeling, Thinking, and Deliberation. Empathy and Democracy: Feeling, Thinking, and Deliberation. University Park: Pennsylvania State University Press.

Mouffe, Chantal. 1996. "Democracy, Power, and the Political." In Democracy \& Difference, ed. Benhabib, Seyl. Princeton, NJ: Princeton University Press. 
Mouffe, Chantal. 2000. The Democratic Paradox. New York: Verso.

Mutz, Diana C. 2002. "Cross-cutting Social Networks: Testing Democratic Theory in Practice." American Political Science Review 96: 1, pp. 111-126.

--------. 2006. Hearing the Other Side: Deliberative Versus Participatory Democracy. Cambridge: Cambridge University Press.

Neblo, Michael, et al. 2010. "Who Wants to Deliberate - and why?" American Political Science Review 104: 3, pp. 566-583.

Nussbaum, Martha. 1995. Poetic Justice: The Literary Imagination and Public Life. Boston: Beacon Press.

Obama, Barack. 2012. "Robin Roberts ABC News Interview with President Obama."

Plato. Crito. 2000. Translated by G.M.A. Grube. In The Trial and Death of Socrates. Indianapolis: Hackett Publishing Company.

--------. Republic. 1969. Edited by Edith Hamilton and Huntington Cairns. Translated by G.M.A. Grube. In The Collected Dialogues of Plato Including the Letters. Princeton: Princeton University Press.

Portman, Rob. 2013. "Rob Portman Commentary: Gay Couples Also Deserve Chance to Get Married." Columbus Dispatch. March 15, 2013.

Prinz, Jesse. 2011. “Against Empathy.” The Southern Journal of Philosophy 29, pp. 214-233.

--------. 2011. “Is Empathy Necessary for Morality?” In Empathy: Philosophical and Psychological Perspectives, eds. Amy Coplan and Peter Goldie. Oxford: Oxford University Press.

Rawls, John. 1971. A Theory of Justice: Original Edition. Cambridge, MA: Hardvard Univeristy Press.

--------. 2005 [1993]. Political Liberalism. New York: Columbia University Press.

--------. 1997. “The Idea of Public Reason Revisited.” The University of Chicago Law Review, $64: 3$, pp. $765-807$.

Rorty, Richard. 1989. Contingency, Irony, and Solidarity. Cambridge: Cambridge University Press.

Sanders, Lynn. 1997. “Against Deliberation.” Political Theory, 25: 3, pp. 347-76. 
Shapiro, Ian. 2003. The State of Democratic Theory. Princeton: Princeton University Press.

Silvers, Anita. 1994. "'Defective' Agents: Equality, Difference and the Tyranny of the Normal." Journal of Social Philosophy 25 (1): 154-75.

Stow, Simon. 2007. Republic of Readers? The Literary Turn in Political Thought and Analysis. Albany NY: SUNY Press.

Titchener, Edward B. 1915. A Beginner's Psychology. New York: Macmillan.

Von Boven, Leaf, and George Loewenstein. 2005. "Empathy Gaps in Emotional Perspective Taking." Other Minds: How Humans Bridge the Divide Between Self and Others, ed. Betram Malle and Sara Hodges, 284-297. New York: The Guilford Press.

White, Stephen K., and Evan Robert Farr. 2011. “'No-Saying' in Habermas.” Political Theory 40: 1, pp. 32-57.

White, Stephen K. 1991. Political Theory and Post Modernism. Cambridge: Cambridge University Press.

------. 1994. Edmund Burke: Modernity, Politics, and Aesthetics. Thousand Oaks, CA: SAGE Publications.

------. 1995. The Cambridge Companion to Habermas. Cambridge: Cambridge University Press.

------. 2009. The Ethos of a Late Modern Citizen. Cambridge, MA: Harvard University Press.

West, Robin. 2013. "The Anti-Empathic Turn.” In Nomos LIII: Passions and Emotions, ed. James E. Fleming. New York: NYU Press.

Young, Iris Marion. 1990. Justice and the Politics of Difference. Princeton, NJ: Princeton Univeristy Press.

-------. 1997A. "Difference as a Resource in Democratic Deliberation.” In Deliberative Democracy: Essays on Reason and Politics, eds. James Bohman and William Rehg. Cambridge, MA: MIT Press, pp. 383-406.

------. 1997B. Intersecting Voices: Dilemmas of Gender, Political Philosophy, and Policy. Princeton: Princeton University Press.

------. 2000. Inclusion and Democracy. Oxford: UK: Oxford University Press.

------. 2001. “Asymmetrical Reciprocity: On Moral Respect, Wonder, and Enlarged Thought.” In Judgment, Imagination, and Politics, ed. Ronald Beiner and Jennifer Nedelsky. Lanham, Md: Rowman and Littlefield, pp. 205-28. 
